\title{
Atomic data for Ne-like ions useful in Plasma Diagnostic
}

\begin{tabular}{|r|l|}
\hline Journal: & Canadian Journal of Physics \\
\hline Manuscript ID & cjp-2017-0031.R2 \\
\hline Manuscript Type: & Article \\
\hline Date Submitted by the Author: & 14-Jun-2017 \\
\hline Complete List of Authors: & $\begin{array}{l}\text { Singh, Narendra; Delhi University, } \\
\text { Aggarwal, Sunny; University of Delhi, Dept. Of physics and astrophysics } \\
\text { Kehan, Man; University of Delhi, Department of Physics }\end{array}$ \\
\hline $\begin{array}{r}\text { Is the invited manuscript for } \\
\text { consideration in a Special } \\
\text { Issue? : }\end{array}$ & N/A \\
\hline &
\end{tabular}

SCHOLARONEm

Manuscripts 


\title{
Atomic data for Ne-like ions useful in Plasma Diagnostic
}

Narendra Singh ${ }^{1}$, Sunny Aggarwal ${ }^{1}$ and Man Mohan ${ }^{2}$

${ }^{1}$ Department of Physics Shyamlal College, University of Delhi, Delhi-110 032 India.

${ }^{2}$ Department of Physics \& Astrophysics, University of Delhi, Delhi-110 007 India. nsingh76@yahoo.co.in

\begin{abstract}
Motived by the recent measurement of transition lines for Ne-like Hf and W, we have reported atomic data in form of multiconfiguration Dirac-Fock transition energies and wavefunction compositions of 209 levels belonging to the configurations $2 s^{2} 2 p^{6}, 2 s^{2} 2 p^{5} n s$ $(n=3,4,5,6,7), 2 s^{2} 2 p^{5} n p(n=3,4,5,6,7), 2 s^{2} 2 p^{5} n d(n=3,4,5,6,7), 2 s^{2} 2 p^{5} n f(n=4,5), 2 s^{2} 2 p^{5} 5 g$, $2 s 2 p^{6} n s(n=3,4,5), 2 s 2 p^{6} n p(n=3,4,5), 2 s 2 p^{6} n d(n=3,4,5), 2 s 2 p^{6} n f(n=4,5)$ and $2 s^{2} 2 p^{6} 5 g$ of Hf LXIII, Ta LXIV, W LXV and Re LXVI. Radiative rates, oscillator strengths, transition wavelengths and line strengths have been calculated for ground state electric dipole (E1) transition among these levels. These values were obtained using GRASP (general-purpose relativistic atomic structure package) code which includes Breit and QED effects along with Dirac-Fock potential and second order coulomb interaction. We have compared our results with the data complied using FAC (Flexible Atomic Code) and also with the recent results available in the literature. The accuracy of the data is assessed. We predict new energy levels, oscillator strength and transition probability data, where no other theoretical or experimental results are available, which will form the basis for future experimental work.
\end{abstract}

PACS. 31.15.ag 


\section{Introduction}

Atomic physics has played a significant role in the history of plasma physics to understand the plasma energy balance and diagnostic development. The atomic physics of tungsten has become of high importance as tungsten will be a constituent of International Thermonuclear Energy Reactor (ITER) plasma. ITER diagnostics has developed using tungsten radiation. The core imaging X-ray spectrometer (CIXS) is a diagnostic designed to measure ion temperature and bulk plasma motion of ITER's core. It has been designed to focus on the Xray emission of neon-like tungsten ions which is included in the present work. The Spectrum of neon-like tungsten near $8500 \mathrm{eV}$ has been measured by Beiersdorfer et al. [1] which require the reliable calculations for comparison. This is because the CIXS will provide radial profiles of the ion abundances in ITER and therefore reliable atomic data is required for using the CIXS in this endeavor. Further, the transition lines have also been measured for Ne-like Hf [2]. Therefore, to provide support to experimentalists and to extend the database, we have calculated the reliable atomic data in form of energy level, oscillator strength, transition probability and line strength for Ne-like ions $(Z=72-75)$.

Ions of Ne-isoelectronic sequence have been studied both experimentally and theoretically over a wide range of $Z$. But nevertheless the study of Ne-like ions remains of interest as spectra of these ions are the source of many important diagnostics informations on plasma such as electron temperature, electron density and charge state abundance. Ne-like ions of middle and high atomic number are present in tokamak, laser produced plasma, EBIT, solar atmosphere [3-4]. Also, Ne-like ions are highly useful for the modelling of astrophysical, fusion and laser generated plasma. Further, because of their closed shell structure, we can estimate the importance of the contributions from relativistic, electron correlations and quantum electrodynamics (QED) effects in study of energy values and radiative rates particularly, transitions between levels of $2 p^{5} 3 s, 3 p$ and $3 d$ configurations 
produce prominent lines in the spectra of high temperature light source in Ne-like ions [5-6]. To obtain laser action the $3 \mathrm{~s}$ and $3 \mathrm{p}$ states have utilized. Also, laser is obtained in lighter elements [7] for Ne-like ions.

Many investigations have been carried out for the Ne-like ions considered in the present work in the last two decades. On the experimental side, many experiments have been performed for the lifetimes of the 31 configurations of Ne-like ions using beam foil method [8-11]. Many authors calculated the transition energies, oscillator strength, lifetimes and transition probabilities using different theoretical methods for many Ne-like ions. [12-17]. Recently, Beiersdorfer et. al.[18] reported radiative rates for transitions in Ne-like ions $(Z=10-110)$. Much work has been done on Ne-like $\mathrm{W}$ due to its importance in exploring the physical conditions in tokamak plasmas such as ITER. In W LXV, Aglitskii et al. [19] calculated energy levels for Ne-like ions from Kr XXVII to U LXXXIII using semiempirical adjusted relativistic calculations. After this, several x-ray lines were precisely measured using EBIT [20-22]. Recently, Khatri et al. [23] calculated photoioization cross section of Ne-like tungsten using R-matrix method. More recently, Aggarwal and keenan [24] calculated energy levels and radiative rates for 121 levels of W LXV.

In this work, we have reported transition energies and wavefunction compositions of 209 levels belonging to the configurations $2 s^{2} 2 p^{6}, 2 p^{5} n s(n=3,4,5,6,7), 2 p^{5} n p(n=3,4,5,6,7)$, $2 p^{5}$ nd $(n=3,4,5,6,7), 2 p^{5} n f(n=4,5), 2 p^{5} 5 g, 2 s 2 p^{6} n s(n=3,4,5), 2 s 2 p^{6} n p \quad(n=3,4,5), 2 s 2 p^{6} n d$ $(n=3,4,5), 2 s 2 p^{6} n f(n=4,5)$ and $2 s^{2} p^{6} 5 g$ of Hf LXIII, Ta LXIV, W LXV and Re LXVI. We have also taken into account Breit and QED effects. Radiative rates, oscillator strengths, transition wavelengths and line strengths are also calculated for all electric dipole (E1), magnetic dipole (M1), electric quadrupole (E2) and magnetic quadrupole (M2) transitions among these levels. We have obtained results using the multiconfiguration Dirac-Fock 
(MCDF) method employed in the GRASP code of Grant et al. [25], revised by Norrington [26]. The calculations are helpful in analyzing new data from many different plasma.

\section{Energy Levels}

In present paper, we have used extensive configuration interaction employed in General purpose relativistic atomic structure package (GRASP) code. GRASP is a fully relativistic code which include Breit and QED correction. We have used GRASP0 in present calculations, which was originally developed by Grant et al. [25] and modified by Norrington [26]. We have used Extended Average Level (EAL) option to optimize the MCDF orbital in present calculations. In these calculations we have taken 64 configurations $\left(2 s^{2} 2 p^{6}, 2 s^{2} 2 p^{5} 31\right.$ $(l=0-2), 2 s^{2} 2 p^{5} 41(l=0-3), 2 s^{2} 2 p^{5} 51(l=0-4), 2 s 2 p^{6} 31 \quad(l=0-2), 2 s 2 p^{6} 41(l=0-3), 2 s 2 p^{6} 51(1=0-4)$, $2 s^{2} 2 p^{5} 61(1=0-2), 2 s^{2} 2 p^{5} 71(1=0-2), 2 s^{2} 2 p^{4} 3 s 3 p, 2 s^{2} 2 p^{4} 3 s 3 d, 2 s^{2} 2 p^{4} 3 p 3 d, 2 s^{2} 2 p^{4} 3 s^{2}, 2 s^{2} 2 p^{4} 3 p^{2}$, $2 s^{2} 2 p^{4} 3 d^{2}, \quad 2 s^{2} 2 p^{4} 3 s 4 s, \quad 2 s^{2} 2 p^{4} 3 s 4 p, \quad 2 s^{2} 2 p^{4} 3 s 4 d, \quad 2 s^{2} 2 p^{4} 3 s 4 f, \quad 2 s^{2} 2 p^{4} 3 s 5 s, \quad 2 s^{2} 2 p^{4} 3 s 5 p$, $2 s^{2} 2 p^{4} 3 s 5 d, \quad 2 s^{2} 2 p^{4} 3 s 5 f, \quad 2 s^{2} 2 p^{4} 3 s 5 g, \quad 2 s^{2} 2 p^{4} 3 p 4 s, \quad 2 s^{2} 2 p^{4} 3 p 4 p, \quad 2 s^{2} 2 p^{4} 3 p 4 d, \quad 2 s^{2} 2 p^{4} 3 p 4 f$, $2 s^{2} 2 p^{4} 3 p 5 s, \quad 2 s^{2} 2 p^{4} 3 p 5 p, \quad 2 s^{2} 2 p^{4} 3 p 5 d, \quad 2 s^{2} 2 p^{4} 3 p 5 f, \quad 2 s^{2} 2 p^{4} 3 p 5 g, \quad 2 s^{2} 2 p^{4} 3 d 4 s, \quad 2 s^{2} 2 p^{4} 3 d 4 p$, $\left.2 s^{2} 2 p^{4} 3 d 4 d, 2 s^{2} 2 p^{4} 3 d 4 f, 2 s^{2} 2 p^{4} 3 d 5 s, 2 s^{2} 2 p^{4} 3 d 5 p, 2 s^{2} 2 p^{4} 3 d 5 d, 2 s^{2} 2 p^{4} 3 d 5 f, 2 s^{2} 2 p^{4} 3 d 5 g\right)$ which generate 3948 fine structure levels, out of which we have presented energy values of 209 fine structure levels belonging to the configurations $\left(2 s^{2} 2 p^{6}, 2 p^{5} n s \quad(n=3,4,5,6,7), 2 p^{5} n p\right.$ $(n=3,4,5,6,7), 2 p^{5} n d(n=3,4,5,6,7), 2 p^{5} n f(n=4,5), 2 p^{5} 5 g, 2 s 2 p^{6} n s(n=3,4,5), 2 s 2 p^{6} n p(n=3,4,5)$, $2 \mathrm{~s} 2 \mathrm{p}^{6} \mathrm{nd}(\mathrm{n}=3,4,5), 2 \mathrm{~s} 2 \mathrm{p}^{6} \mathrm{nf}(\mathrm{n}=4,5)$ and $\left.2 \mathrm{~s} 2 \mathrm{p}^{6} 5 \mathrm{~g}\right)$ of Ne like Hf, W, Ta and Re. We have also performed similar calculations using an independent method flexible atomic code (FAC), which is also a fully relativistic code and give the comparable results with GRASP. We have successfully applied GRASP and FAC in past to calculate atomic data for many highly charged ion [27-33]

In table 1-4, we have presented the MCDF energies, lifetimes, and mixing coefficients for Ne like ions $(Z=72-75)$. In the mixing coefficients, we first give the percentage of the 
basis state corresponding to levels name in LS coupling then the second largest percentage together with the related basis and so on. We would like to mention that mixing in our calculations among the levels is very high, for example, we found that $47.8 \%$ of $2 \mathrm{~s}^{2} 2 \mathrm{p}^{5} 7 \mathrm{~d}^{3} \mathrm{P}_{2}$ level is mixed with $35.6 \%$ of $2 \mathrm{~s}^{2} 2 \mathrm{p}^{5} 7 \mathrm{~d}^{3} \mathrm{D}_{2}$. There are many other levels which are mixed. Therefore, the identification of particular level is not unique, although the Aggarwal [24] and Vilkas et al. [34] have the same labels as in our work for Ne like Tungsten.

In table 1-4, we have also presented the energy values for 209 fine structural levels calculated using GRASP code. We have provided the detailed comparison of our energy values calculated from GRASP with the available data compiled by NIST and calculated values from flexible atomic code (FAC) which is independent and fully relativistic atomic structure code. However, NIST energy values are available only for Ne-like Tungsten. Our calculated results from GRASP and FAC are in close agreement with each other for all ions taken in present work. Difference between two sets (FAC and GRASP) are up to 0.5 Ryd. for most of the levels, except for some level for which discrepancies is slightly larger up to 1 Ryd. However, the ordering are almost identical for both calculations for Ne like Tungsten. We have compared energy values calculated from MCDF and FAC with the recent results of Aggarwal [24] and Vilkas [34]. Aggarwal [24] used the same GRASP0 code which is used in our present calculations. Aggarwal et al. [24] reported energies for 121 fine structural levels and calculated energy value only for 157 level. Vilkas et al [34] calculated energies for 141 levels using relativistic multi-reference-many body Moller-Plesset (MRMP) Perturbation theory. These results are listed in table 3 for comparison. In general, our calculated values using GRASP and FAC is closed to NIST. The energy differs only by 0.6 ryd. with our calculated values of GRASP and FAC. Overall, there is no significant discrepancy between the four independent calculations. However, we have listed energy values for 209 fine structural levels which have been presented for the first time. We have presented results for 
many new levels which are not listed in NIST tables and may form the basis of future experimental works.

\section{Radiative Rates}

Apart from energy levels, calculations have also been made for transition wavelength $(\lambda \mathrm{ji})$, of oscillator strength $\left(f_{i j}\right)$, transition probability $\left(A_{i j}, s^{-1}\right)$, line strength $\left(S_{i j}\right.$ in A.U.) for neon like ions $(Z=72-75)$. The transition probability and oscillator strength can be related with line strength as

For the electric dipole (E1) transitions

$$
A_{j i}=\frac{2.0261 \times 10^{18}}{w_{j} \lambda_{j i}^{3}} s \text { and } f_{i j}=\frac{303.75}{\lambda_{j i} w_{i}}
$$

For the magnetic dipole (M1) transitions

$$
A_{j i}=\frac{2.6974 \times 10^{13}}{w_{j} \lambda_{j i}^{3}} s \text { and } f_{i j}=\frac{4.044 \times 10^{-3}}{\lambda_{j i} w_{i}}
$$

For the electric quadrupole (E2) transitions

$$
A_{j i}=\frac{1.1199 \times 10^{18}}{w_{j} \lambda_{j i}^{5}} s \text { and } f_{i j}=\frac{167.89}{\lambda_{j i}{ }^{3} w_{i}}
$$

For the magnetic quadrupole (M2) transitions

$$
A_{j i}=\frac{1.4910 \times 10^{13}}{w_{j} \lambda_{j i}^{5}} s \text { and } f_{i j}=\frac{2.236 \times 10^{-3}}{\lambda_{j i}{ }^{3} w_{i}}
$$

In these equations, $\omega_{i}$ and $\omega_{j}$ are the statistical weights of the lower $i$ and upper $j$ levels respectively.

In table 5-8, we have reported the transition wavelength $(\AA)$, transition probability ( $\left.A_{j i}, s^{-1}\right)$, oscillator strength $\left(f_{i j}\right)$ and line strength $\left(s_{i j}\right.$, in $\left.a . u\right)$ for all electric dipole (E1) transitions from ground state among 209 levels of Ne-like ions $(Z=72-75)$. We have also 
presented the transition wavelength $(\AA)$, transition probability $\left(A_{j i}, s^{-1}\right)$, oscillator strength $\left(f_{i j}\right)$ and line strength $\left(s_{i j}\right.$, in $\left.a . u\right)$ for the transitions within the ground state of Ne-like $\mathrm{W}$ for E2, M1 and M2 in Table 9. We have result of all transitions with us and one can get these results if required. For these calculations, we have used GRASP energies which includes Breit and QED effects. In table 5-8 $\mathrm{j}$ and $\mathrm{i}$ represent the upper and lower level respectively. We have also reported the ratio of velocity and length forms of the oscillator strength because it gives an indication of the accuracy of the $\mathrm{f}$ (or A) values. The good agreement between the two forms is desirable, but not a sufficient condition for accuracy, as different sets of configuration can give same ratio of velocity and length but different result in magnitude. In present calculations one can see from table 5-8, the velocity length ratio is generally within $10 \%$ for strong E1 type transitions. However, difference between two forms for some weaker transitions sometimes be large. All such weak transitions, do not affect the overall accuracy of the calculations.

In table-10, we have compared the calculated transition probability $\left(A_{j i}\right)$, oscillator strength $\left(f_{i j}\right)$ using MCDF method with the earlier calculated results and experimental results $[24,34]$. One can see from table 10 that our calculated results match well with other results, which shows the accuracy of our calculated results.

\section{Lifetimes}

The lifetime for a particular level $\mathrm{j}$ can be defined as follows

$$
\tau_{j}=\frac{1}{\sum_{j} A_{j i}}
$$

In table 1-4, we have presented our calculated lifetime with the GRASP code, which include A-values contributions from Electric dipole (E1), Magnetic dipole (M1), Electric quadrupole (E2) and magnetic quadrupole (M2) transitions. For comparison, theoretical results Aggarwal and Keenan [24] are available for 121 fine structural levels based on calculations with the 
same GRASP0 code. One can see from Table 11 that our calculated lifetime with the GRASP0 code shows excellent agreement with the results of Aggarwal and Keenan [24]. We hope our calculated lifetime will be useful to experimentalist for their future work.

\section{Conclusion}

In present work, energy levels and radiative rates for E1, E2, M1, M2 transitions calculated with GRASP0 code have been reported for the 209 levels of Ne like ions $(Z=72-75)$. We have taken into account the various checks like agreement of calculations by performing similar calculations using FAC code. We have performed accuracy test by comparing our calculated results with the other available results. We have also presented the lifetimes of all excited states. Based on the comparison of several calculations with the two independent codes (GRASP and FAC) and with the other available results, our energy levels are estimated to be accurate to 0.5 Ryd. Also, based on the comparison between velocity and length ratio, we conclude that our radiative rates are accurate to better than $20 \%$ for most of strong transitions. 


\section{References}

[1] P. Beiersdorfer, J. Clementson, and U. Safronova. Atoms, 3, 260 (2015).

[2] P. Beiersdorfer, G.V. Brown, et al. Review of scientific Instruments, 83, 10E111 (2012).

[3] P. Beiersderfor, et al. Phys. Rev. A, 41, 3453 (1990).

[4] D.D. Dietrich et al. Phys. Rev. A, 41, 1450 (1990).

[5] C. Jupen. Nucl. Instr. Meth. B, 31, 166 (1988).

[6] P. Beiersderfor et al. J. Phys. B, 43, 144008 (2010).

[7] J. Nilson, B.J. Macgowan, L.B. Dasilva, and J.C. Moreno. Phys. Rev. A, 48, 4682 (1993).

[8] E. Trabert. Z. Phys. A, 319, 25 (1984).

[9] H.G. Berry, J. Desesquelles, K.T. Cheng, and R.M. Schectman. Phys. Rev. A, 18, 546 (1978).

[10] E. Trabert, and C. Jupen. Phys. Scr., 30, 586 (1987).

[11] Westerlind et al. Phys. Scr., 38, 821, (1988).

[12] C.F. Fischer, and G. Tachiev. At. Mol. Nucl. Data Tables, 87, 1 (2004).

[13] G.X. Chen, A.K. Pradhan, and W. Eissner. J. Phys. B: At. Mol. Opt. Phys., 36, 453 (2003).

[14] S.N. Nahar, W. Eissner, G.X. Chen, and A.K. Pradhan. Astron. Astrophys., 408. 789 (2003).

[15] P. Quinet, T. Gorlia, and E. Biemont. Phys. Scr., 44, 164 (1991).

[16] C.Z. Dong, L.Y. Xie, X.X. Zhou, X.W. Ma, and S. Fritzsche. Hyperfine Interact., 146, 161, (2003).

[17] I.M. Savukov. J. Phys. B: At. Mol. Opt. Phys., 36, 4789 (2003). 
[18] P. Beiersderfor P et al. Phys. Rev. A, 86, 012509 (2012).

[19] E.V. Aglitskii, E.P. Ivanova, S.A. Panin, U.I. Safronova, S.I. Ulityn, L.A. Vainshtein, and

J.F. Wyart. Phys. Scripta, 40, 601 (1989).

[20] P. Beiersdorfer. Proceedings of the Sixth International Conference on the Physics of Highly Charged Ions, Manhattan, KS, USA, AIP Conf. Proc., 274, 365 (1993).

[21] J. Asada. Phys. Scripta, T73, 90 (1997).

[22] C. Biedermann, A. Förster, G. Fußmann, and R. Radtke. Phys. Scripta, T73, 360 (1997).

[23] I. Khatri, A. Goyal, S. Aggarwal, A.K. Singh, and M. Mohan. Can. J. Phys., 93, 1221 (2015).

[24] K.M. Aggarwal, and F.P. Keenan. At. Data Nuclear data Tables, 11, 187 (2016).

[25] I.P. Grant, J. McKenzie, P.H. Norrington, D.F. Mayers, and N.C. Pyper. Comput. Phys. Common., 21, 207 (1980).

[26] P.H. Norrington. 2009 http://www.am.qub.ac.uk/DARC

[27] S. Aggarwal, J. Singh, and M. Mohan. Chin. Phys. B, 22, 033201 (2013).

[28] I. Khatri, A. Goyal, S. Aggarwal, A.K. Singh, and M. Mohan. Radiation physics and Chemistry, 123, 46 (2016).

[29] A.K. Singh, A. Goyal, I. Khatri, S. Aggarwal, R. Sharma, and M. Mohan. Atomic data Nuclear data tables, 109, 339 (2016).

[30] A. Goyal, I. Khatri, S. Aggarwal, A.K. Singh, and M. Mohan M. Atomic data Nuclear data tables, 107, 406 (2016).

[31] I. Khatri, A. Goyal, S. Aggarwal, A.K. Singh, and M. Mohan. Atomic data Nuclear data tables, 107, 367 (2016).

[32] S. Aggarwal, J. Singh, A.K.S. Jha, and M. Mohan. Atomic data Nuclear data tables, 100, 859 (2014). 
[33] S. Aggarwal, J. Singh, and M. Mohan. Atomic data Nuclear data tables, 99, 704 (2013).

[34] M.J. Vilkas, J.M. L'opez-Encarnaci'on, and Y. Ishikawa. At. Data Nucl. Data Tables, 94, 50 (2008).

Table 1: MCDF energies (in Ryd.), lifetimes (in s) and mixing coefficients in Ne-like-Hf

\begin{tabular}{|c|c|c|c|c|c|c|}
\hline S.No & Label & J & $\begin{array}{l}\text { Level } \\
\text { (Ryd) }\end{array}$ & FAC & Lifetimes & Mixing coefficients \\
\hline 1 & $2 s^{2} 2 p^{6}{ }^{1} s^{e}$ & 0 & -------- & 0 & ב-ב--- & 100 \\
\hline 2 & $2 s^{2} 2 p^{5} 3 s^{3} p^{0}$ & 2 & 575.746687 & 575.8738 & $2.99 \mathrm{E}-10$ & 100 \\
\hline 3 & $2 s^{2} 2 p^{5} 3 s^{1} p^{0}$ & 1 & 576.34331 & 576.47 & $7.41 \mathrm{E}-15$ & $66.26+33.76(19)$ \\
\hline 4 & $2 s^{2} 2 p^{5} 3 p^{3} p^{e}$ & 1 & 586.363216 & 586.3716 & $1.81 \mathrm{E}-11$ & $49.28+31.7(24)$ \\
\hline 5 & $2 s^{2} 2 p^{5} 3 p^{3} D^{e}$ & 2 & 586.499437 & 586.5063 & $1.23 \mathrm{E}-12$ & $50.27+33.18(23)+16.48(8)$ \\
\hline 6 & $2 s^{2} 2 p^{5} 3 p^{1} p^{e}$ & 1 & 610.844174 & 610.8702 & $8.08 \mathrm{E}-13$ & $55.95+35.88(24)$ \\
\hline 7 & $2 s^{2} 2 p^{5} 3 p^{3} D^{e}$ & 3 & 610.815701 & 610.8944 & $7.68 \mathrm{E}-13$ & 100 \\
\hline 8 & $2 s^{2} 2 p^{5} 3 p^{3} p^{e}$ & 2 & 611.742975 & 611.7868 & 4.64E-13 & $66.75+33.18(23)$ \\
\hline 9 & $2 s^{2} 2 p^{5} 3 p^{1} s^{e}$ & 0 & 615.870615 & 615.8376 & $5.11 \mathrm{E}-13$ & $61.78+38.07(21)$ \\
\hline 10 & $2 s^{2} 2 p^{5} 3 d^{3} p^{0}$ & 0 & 622.13175 & 622.1152 & $1.04 \mathrm{E}-12$ & 100 \\
\hline 11 & $2 s^{2} 2 p^{5} 3 d^{3} p^{0}$ & 1 & 622.837977 & 622.8201 & $1.54 \mathrm{E}-14$ & $66.75+31.81(29)$ \\
\hline 12 & $2 s^{2} 2 p^{5} 3 d^{3} F^{0}$ & 3 & 622.926827 & 622.9091 & $9.82 \mathrm{E}-13$ & $54.02+39.06(31)$ \\
\hline 13 & $2 s^{2} 2 p^{5} 3 d^{3} D^{0}$ & 2 & 623.491863 & 623.4683 & $9.34 \mathrm{E}-13$ & $54.61+18.23(28)+17.31(15)$ \\
\hline 14 & $2 s^{2} 2 p^{5} 3 d^{3} F^{0}$ & 4 & 628.273596 & 628.2818 & $7.31 \mathrm{E}-12$ & 100 \\
\hline 15 & $2 s^{2} 2 p^{5} 3 d^{1} D^{0}$ & 2 & 628.776936 & 628.7669 & 4.19E-12 & $48.72+41.09(30)$ \\
\hline 16 & $2 s^{2} 2 p^{5} 3 d^{3} D^{0}$ & 3 & 629.497131 & 629.4815 & $6.65 \mathrm{E}-12$ & $70.56+27.35(31)$ \\
\hline 17 & $2 s^{2} 2 p^{5} 3 d^{1} p^{0}$ & 1 & 631.617571 & 631.5568 & 4.04E-16 & $62.88+19.45(29)+17.64(11)$ \\
\hline 18 & $2 s^{2} 2 p^{5} 3 s^{3} p^{0}$ & 0 & 665.501858 & 665.707 & 4.53E-11 & 99.8 \\
\hline 19 & $2 s^{2} 2 p^{5} 3 s^{3} p^{0}$ & 1 & 665.746963 & 665.952 & $3.01 \mathrm{E}-14$ & $66.1+33.76(3)$ \\
\hline 20 & $2 s^{2} 2 p^{5} 3 p^{3} D^{e}$ & 1 & 675.858759 & 675.9504 & $1.84 \mathrm{E}-11$ & $73.62+22.75(6)$ \\
\hline 21 & $2 s^{2} 2 p^{5} 3 p^{3} p^{e}$ & 0 & 679.339609 & 679.3984 & $3.53 \mathrm{E}-12$ & $61.78+37.95(9)$ \\
\hline 22 & $2 s 2 p^{6} 3 s^{3} s^{e}$ & 1 & 698.923001 & 698.9252 & $1.19 \mathrm{E}-13$ & $62.09+19.89(4)$ \\
\hline 23 & $2 s^{2} 2 p^{5} 3 p^{1} D^{e}$ & 2 & 700.888993 & 701.0201 & 4.43E-13 & $49.7+33.52(23)+16.81(8)$ \\
\hline 24 & $2 s^{2} 2 p^{5} 3 p^{3} s^{e}$ & 1 & 702.228474 & 702.0981 & $8.52 \mathrm{E}-14$ & $37.82+30.69(4)+19.18(24)$ \\
\hline 25 & $2 s 2 p^{6} 3 s^{1} s^{e}$ & 0 & 702.816187 & 702.4415 & $5.95 \mathrm{E}-14$ & 99.8 \\
\hline 26 & $2 s 2 p^{6} 3 p^{3} p^{0}$ & 1 & 710.791342 & 710.5377 & $9.92 \mathrm{E}-16$ & $64+30.91(33)$ \\
\hline 27 & $2 s 2 p^{6} 3 p^{3} p^{0}$ & 0 & 711.101696 & 710.7513 & $5.59 \mathrm{E}-14$ & 99.8 \\
\hline 28 & $2 s^{2} 2 p^{5} 3 d^{3} F^{0}$ & 2 & 712.6381 & 712.7137 & $9.40 \mathrm{E}-13$ & $74.13+20.43(15)$ \\
\hline 29 & $2 s^{2} 2 p^{5} 3 d^{3} D^{0}$ & 1 & 714.74448 & 714.6845 & $1.12 \mathrm{E}-15$ & $45.97+33.76(17)$ \\
\hline 30 & $2 s^{2} 2 p^{5} 3 d^{3} p^{0}$ & 2 & 718.467536 & 718.5346 & 4.04E-12 & $46.92+36.12(13)$ \\
\hline 31 & $2 s^{2} 2 p^{5} 3 d^{1} F^{0}$ & 3 & 718.716558 & 718.7858 & $6.13 \mathrm{E}-12$ & $43.96+33.52(31)+22.47(16)$ \\
\hline 32 & $2 s 2 p^{6} 3 p^{3} p^{0}$ & 2 & 735.687686 & 735.3904 & $5.19 \mathrm{E}-14$ & 99.6 \\
\hline 33 & $2 s 2 p^{6} 3 p^{1} p^{0}$ & 1 & 736.114445 & 735.814 & $2.39 \mathrm{E}-15$ & $67.9+32.04(26)$ \\
\hline 34 & $2 s 2 p^{6} 3 d^{3} D^{e}$ & 1 & 747.45884 & 747.037 & $5.78 \mathrm{E}-14$ & 100 \\
\hline 35 & $2 s 2 p^{6} 3 d^{3} D^{e}$ & 2 & 747.840811 & 747.4183 & 4.47E-14 & $71.4+28.52(37)$ \\
\hline 36 & $2 s 2 p^{6} 3 d{ }^{3} D^{e}$ & 3 & 753.077877 & 752.6785 & $5.72 \mathrm{E}-14$ & 100 \\
\hline 37 & $2 s 2 p^{6} 3 d^{1} D^{e}$ & 2 & 754.186817 & 753.7721 & 3.74E-14 & $71.4+28.52(35)$ \\
\hline 38 & $2 s^{2} 2 p^{5} 4 s^{3} p^{0}$ & 2 & 804.548061 & 804.6512 & 1.67E-14 & 100 \\
\hline 39 & $2 s^{2} 2 p^{5} 4 s^{1} p^{0}$ & 1 & 804.748696 & 804.8524 & $9.27 \mathrm{E}-15$ & $66.59+33.41(63)$ \\
\hline 40 & $2 s^{2} 2 p^{5} 4 p^{3} p^{e}$ & 1 & 808.934454 & 808.9941 & $1.38 \mathrm{E}-14$ & $49.7+31.25(70)$ \\
\hline 41 & $2 s^{2} 2 p^{5} 4 p^{3} D^{e}$ & 2 & 808.973978 & 809.034 & $1.37 \mathrm{E}-14$ & $50.13+33.41(71)+16.48(44)$ \\
\hline 42 & $2 s^{2} 2 p^{5} 4 p^{3} D^{e}$ & 3 & 818.974686 & 819.0525 & $2.10 \mathrm{E}-14$ & 100 \\
\hline 43 & $2 s^{2} 2 p^{5} 4 p^{1} p^{e}$ & 1 & 818.997244 & 819.0737 & $2.09 \mathrm{E}-14$ & $56.4+35.76(70)$ \\
\hline 44 & $2 s^{2} 2 p^{5} 4 p^{3} p^{e}$ & 2 & 819.316808 & 819.3925 & $2.11 \mathrm{E}-14$ & $66.75+33.18(71)$ \\
\hline 45 & $2 s^{2} 2 p^{5} 4 p^{1} s^{e}$ & 0 & 820.721973 & 820.7369 & $2.18 \mathrm{E}-14$ & $65.45+34.46(65)$ \\
\hline 46 & $2 s^{2} 2 p^{5} 4 d^{3} p^{0}$ & 0 & 823.312993 & 823.366 & $9.19 \mathrm{E}-15$ & 100 \\
\hline 47 & $2 s^{2} 2 p^{5} 4 d^{3} p^{0}$ & 1 & 823.568258 & 823.6192 & $7.44 \mathrm{E}-15$ & $64.8+33.29(77)$ \\
\hline 48 & $2 s^{2} 2 p^{5} 4 d^{3} F^{0}$ & 3 & 823.585955 & 823.6352 & $9.23 \mathrm{E}-15$ & $53.58+39.44(83)$ \\
\hline 49 & $2 s^{2} 2 p^{5} 4 d^{3} D^{0}$ & 2 & 823.791622 & 823.837 & $9.28 \mathrm{E}-15$ & $54.46+18.15(75)+17.89(51)$ \\
\hline 50 & $2 s^{2} 2 p^{5} 4 d^{3} F^{0}$ & 4 & 825.921357 & 825.9796 & $8.92 \mathrm{E}-15$ & 100 \\
\hline 51 & $2 s^{2} 2 p^{5} 4 d^{1} D^{0}$ & 2 & 826.107648 & 826.1615 & $8.92 \mathrm{E}-15$ & $48.58+40.96(82)$ \\
\hline 52 & $2 s^{2} 2 p^{5} 4 d^{3} D^{\circ}$ & 3 & 826.365858 & 826.4155 & $8.98 \mathrm{E}-15$ & $70.73+27.14(83)$ \\
\hline 53 & $2 s^{2} 2 p^{5} 4 d^{1} P^{0}$ & 1 & 827.108991 & 827.1346 & $9.92 \mathrm{E}-16$ & $63.84+18.92(47)+17.06(77)$ \\
\hline
\end{tabular}




\begin{tabular}{|c|c|c|c|c|c|c|}
\hline 54 & $2 s^{2} 2 p^{5} 4 f^{3} D^{e}$ & 1 & 828.442392 & 828.5077 & $4.25 \mathrm{E}-15$ & 100 \\
\hline 55 & $2 s^{2} 2 p^{5} 4 f^{3} G^{e}$ & 4 & 828.465363 & 828.5308 & 4.36E-15 & $52.85+44.22(107)$ \\
\hline 56 & $2 s^{2} 2 p^{5} 4 f^{3} F^{e}$ & 2 & 828.613355 & 828.6786 & 4.30E-15 & $47.33+47.06(101)$ \\
\hline 57 & $2 s^{2} 2 p^{5} 4 f^{3} F^{e}$ & 3 & 828.69074 & 828.756 & $4.35 \mathrm{E}-15$ & $50.69+22.75(100)+22.37(60)$ \\
\hline 58 & $2 s^{2} 2 p^{5} 4 f^{3} G^{e}$ & 5 & 829.550736 & 829.6162 & $4.46 \mathrm{E}-15$ & 100 \\
\hline 59 & $2 s^{2} 2 p^{5} 4 f^{1} D^{e}$ & 2 & 829.670818 & 829.7337 & 4.35E-15 & $61+30.8(101)$ \\
\hline 60 & $2 s^{2} 2 p^{5} 4 f^{1} F^{e}$ & 3 & 829.731299 & 829.796 & $4.42 \mathrm{E}-15$ & $44.22+40.45(106)$ \\
\hline 61 & $2 s^{2} 2 p^{5} 4 f^{3} F^{e}$ & 4 & 829.800938 & 829.8662 & 4.47E-15 & $72.08+22.47(107)$ \\
\hline 62 & $2 s^{2} 2 p^{5} 4 s^{3} p^{0}$ & 0 & 894.411874 & 894.6042 & $1.65 \mathrm{E}-14$ & 100 \\
\hline 63 & $2 s^{2} 2 p^{5} 4 s^{3} p^{0}$ & 1 & 894.480913 & 894.6737 & $1.29 \mathrm{E}-14$ & $66.42+33.41(39)$ \\
\hline 64 & $2 s^{2} 2 p^{5} 4 p^{3} D^{e}$ & 1 & 898.694629 & 898.8433 & $1.36 \mathrm{E}-14$ & $73.96+22.37(43)$ \\
\hline 65 & $2 s^{2} 2 p^{5} 4 p^{3} p^{e}$ & 0 & 899.805267 & 899.9265 & $1.41 \mathrm{E}-14$ & $65.45+34.34(45)$ \\
\hline 66 & $2 s^{2} 2 p^{5} 5 s^{3} p^{0}$ & 2 & 906.592421 & 906.7052 & $2.06 \mathrm{E}-14$ & 100 \\
\hline 67 & $2 s^{2} 2 p^{5} 5 s^{1} p^{0}$ & 1 & 906.686483 & 906.7979 & $1.26 \mathrm{E}-14$ & $66.59+33.41(155)$ \\
\hline 68 & $2 s^{2} 2 p^{5} 5 p^{3} p^{e}$ & 1 & 908.622957 & 908.7901 & $1.88 \mathrm{E}-14$ & $48.86+30.8(158)$ \\
\hline 69 & $2 s^{2} 2 p^{5} 5 p^{3} D^{e}$ & 2 & 908.733916 & 908.876 & $1.88 \mathrm{E}-14$ & $49.7+33.29(159)+16.4(74)$ \\
\hline 70 & $2 s^{2} 2 p^{5} 4 p^{3} s^{e}$ & 1 & 909.071993 & 909.1448 & $1.89 \mathrm{E}-14$ & $49.28+28.62(70)$ \\
\hline 71 & $2 s^{2} 2 p^{5} 4 p^{1} D^{e}$ & 2 & 909.032035 & 909.1594 & $1.87 \mathrm{E}-14$ & $49.56+33.29(71)+16.65(44)$ \\
\hline 72 & $2 s^{2} 2 p^{5} 5 p^{3} D^{e}$ & 3 & 913.857016 & 913.5623 & $2.47 \mathrm{E}-14$ & 100 \\
\hline 73 & $2 s^{2} 2 p^{5} 5 p^{1} p^{e}$ & 1 & 913.867478 & 913.9557 & $2.42 \mathrm{E}-14$ & $56.4+35.52(158)$ \\
\hline 74 & $2 s^{2} 2 p^{5} 5 p^{3} p^{e}$ & 2 & 914.024819 & 913.9652 & $2.49 \mathrm{E}-14$ & $66.75+33.18(159)$ \\
\hline 75 & $2 s^{2} 2 p^{5} 4 d^{3} F^{0}$ & 2 & 913.42024 & 914.1121 & $9.24 \mathrm{E}-15$ & $74.3+20.07(51)$ \\
\hline 76 & $2 s^{2} 2 p^{5} 5 p{ }^{1} s^{e}$ & 0 & 914.697871 & 914.1199 & $2.55 \mathrm{E}-14$ & $65.77+33.76(157)$ \\
\hline 77 & $2 s^{2} 2 p^{5} 4 d{ }^{3} D^{0}$ & 1 & 913.982019 & 914.7537 & $1.79 \mathrm{E}-15$ & $48.02+32.72(53)$ \\
\hline 78 & $2 s^{2} 2 p^{5} 5 d^{3} p^{0}$ & 0 & 915.989087 & 916.0403 & $1.23 \mathrm{E}-14$ & 100 \\
\hline 79 & $2 s^{2} 2 p^{5} 5 d^{3} p^{0}$ & 1 & 916.115063 & 916.08 & 1.10E-14 & $63.36+33.99(161)$ \\
\hline 80 & $2 s^{2} 2 p^{5} 5 d^{3} F^{0}$ & 3 & 916.171237 & 916.0818 & 1.17E-14 & $52.85+38.81(163)$ \\
\hline 81 & $2 s^{2} 2 p^{5} 5 d^{3} D^{0}$ & 2 & 916.246206 & 916.2049 & $1.22 \mathrm{E}-14$ & $54.32+17.47(160)+16.4(85)$ \\
\hline 82 & $2 s^{2} 2 p^{5} 4 d^{3} p^{0}$ & 2 & 915.895396 & 916.3013 & $9.03 \mathrm{E}-15$ & $42.12+32.04(49)$ \\
\hline 83 & $2 s^{2} 2 p^{5} 4 d^{1} F^{\circ}$ & 3 & 915.976274 & 916.3422 & $9.39 \mathrm{E}-15$ & $43.43+32.95(83)+21.9(52)$ \\
\hline 84 & $2 s^{2} 2 p^{5} 5 d^{3} F^{0}$ & 4 & 917.326664 & 917.4218 & $1.23 \mathrm{E}-14$ & 100 \\
\hline 85 & $2 s^{2} 2 p^{5} 5 d{ }^{1} D^{0}$ & 2 & 917.446362 & 917.5284 & $1.22 \mathrm{E}-14$ & $42.51+37.82(162)$ \\
\hline 86 & $2 s^{2} 2 p^{5} 5 d^{3} D^{0}$ & 3 & 917.541537 & 917.6284 & $1.23 \mathrm{E}-14$ & $70.56+26.63(163)$ \\
\hline 87 & $2 s^{2} 2 p^{5} 5 d^{1} p^{0}$ & 1 & 917.954051 & 918.0174 & $1.59 \mathrm{E}-15$ & $61.94+18.92(790$ \\
\hline 88 & $2 s^{2} 2 p^{5} 5 f^{3} D^{e}$ & 1 & 918.556013 & 918.6066 & $8.12 \mathrm{E}-15$ & 100 \\
\hline 89 & $2 s^{2} 2 p^{5} 5 f^{3} G^{e}$ & 4 & 918.580667 & 918.6348 & $8.25 \mathrm{E}-15$ & $53+43.82(167)$ \\
\hline 90 & $2 s^{2} 2 p^{5} 5 f^{3} D^{e}$ & 2 & 918.672785 & 918.657 & $7.58 \mathrm{E}-15$ & $47.89+46.51(165)$ \\
\hline 91 & $2 s^{2} 2 p^{5} 5 f^{3} F^{e}$ & 3 & 918.697069 & 918.6813 & $8.02 \mathrm{E}-15$ & $50.55+22.56(164)+22.18(94)$ \\
\hline 92 & $2 s^{2} 2 p^{5} 5 f^{3} G^{e}$ & 5 & 919.13546 & 918.8009 & $8.48 \mathrm{E}-15$ & 100 \\
\hline 93 & $2 s^{2} 2 p^{5} 5 f^{1} D^{e}$ & 2 & 919.211287 & 918.8026 & $8.24 \mathrm{E}-15$ & $61.62+29.05(90)$ \\
\hline 94 & $2 s^{2} 2 p^{5} 5 f{ }^{1} F^{e}$ & 3 & 919.196164 & 919.2365 & $7.78 \mathrm{E}-15$ & $43.3+39.82(166)$ \\
\hline 95 & $2 s^{2} 2 p^{5} 5 f^{3} \mathrm{~F}^{\mathrm{e}}$ & 4 & 919.252662 & 919.3042 & $8.16 \mathrm{E}-15$ & $71.74+22.75(167)$ \\
\hline 96 & $2 s^{2} 2 p^{5} 5 g^{3} F^{\circ}$ & 2 & 919.327175 & 919.3098 & $1.46 \mathrm{E}-14$ & 100 \\
\hline 97 & $2 s^{2} 2 p^{5} 5 g{ }^{3} H^{0}$ & 5 & 919.333579 & 919.3559 & $1.46 \mathrm{E}-14$ & $52.42+45.83(170)$ \\
\hline 98 & $2 s^{2} 2 p^{5} 5 g{ }^{3} G^{0}$ & 3 & 919.378871 & 919.4173 & $1.46 \mathrm{E}-14$ & $54.17+37.09(169)$ \\
\hline 99 & $2 s^{2} 2 p^{5} 5 g{ }^{3} G^{0}$ & 4 & 919.397721 & 919.4234 & $1.46 \mathrm{E}-14$ & $47.61+25.2(168)+24.9(104)$ \\
\hline 100 & $2 s^{2} 2 p^{5} 4 f^{3} G^{e}$ & 3 & 918.456756 & 919.4691 & 4.40E-15 & $72.25+18.66(60)$ \\
\hline 101 & $2 s^{2} 2 p^{5} 4 f^{3} D^{e}$ & 2 & 918.508021 & 919.488 & $4.46 \mathrm{E}-15$ & $44.09+32.95(59)+21.9(101)$ \\
\hline 102 & $2 s^{2} 2 p^{5} 5 g^{1} F^{\circ}$ & 3 & 919.670309 & 919.7529 & $1.48 \mathrm{E}-14$ & $57.91+37.95(169)$ \\
\hline 103 & $2 s^{2} 2 p^{5} 5 g{ }^{3} H^{0}$ & 6 & 919.672183 & 919.7603 & $1.47 \mathrm{E}-14$ & 100 \\
\hline 104 & $2 s^{1} 2 p^{6} 5 g{ }^{1} G^{0}$ & 4 & 919.719734 & 919.762 & $1.48 \mathrm{E}-14$ & $41.73+39.31(171)+17.39(99)$ \\
\hline 105 & $2 s^{1} 2 p^{6} 5 g{ }^{3} G^{0}$ & 5 & 919.735118 & 919.7708 & $1.47 \mathrm{E}-14$ & $71.57+20.79(170)$ \\
\hline 106 & $2 s^{2} 2 p^{5} 4 f^{3} D^{e}$ & 3 & 919.625726 & 919.81 & 4.64E-15 & $54.02+29.7(57)$ \\
\hline 107 & $2 s^{2} 2 p^{5} 4 f^{1} G^{e}$ & 4 & 919.60262 & 919.8254 & 4.56E-15 & $41.47+33.29(107)+25.1(61)$ \\
\hline 108 & $2 s 2 p^{6} 4 s^{3} s^{e}$ & 1 & 928.931364 & 928.7493 & $1.28 \mathrm{E}-14$ & 99.8 \\
\hline 109 & $2 s 2 p^{6} 4 s^{1} s^{e}$ & 0 & 929.712771 & 929.4922 & $1.29 \mathrm{E}-14$ & 100 \\
\hline 110 & $2 s 2 p^{6} 4 p{ }^{3} p^{0}$ & 0 & 933.348591 & 933.1207 & $1.11 \mathrm{E}-14$ & 100 \\
\hline 111 & $2 s 2 p^{6} 4 p^{3} p^{0}$ & 1 & 933.38058 & 933.1526 & $4.62 \mathrm{E}-15$ & $67.57+32.26(113)$ \\
\hline 112 & $2 s 2 p^{6} 4 p^{3} p^{0}$ & 2 & 943.447317 & 943.2421 & $1.54 \mathrm{E}-14$ & 100 \\
\hline 113 & $2 s 2 p^{6} 4 p^{1} p^{0}$ & 1 & 943.598646 & 943.3904 & $3.60 \mathrm{E}-15$ & $67.57+32.28(111)$ \\
\hline 114 & $2 s 2 p^{6} 4 d^{3} D^{e}$ & 1 & 947.946498 & 947.7056 & $7.92 \mathrm{E}-15$ & 100 \\
\hline 115 & $2 s 2 p^{6} 4 d^{3} D^{e}$ & 2 & 948.08087 & 947.8399 & $7.88 \mathrm{E}-15$ & $69.06+30.91(117)$ \\
\hline 116 & $2 s 2 p^{6} 4 d^{3} D^{e}$ & 3 & 950.37505 & 950.1483 & $7.69 \mathrm{E}-15$ & 100 \\
\hline 117 & $2 s 2 p^{6} 4 d^{1} D^{e}$ & 2 & 950.762333 & 950.5233 & $7.61 \mathrm{E}-15$ & $69.06+30.91(115)$ \\
\hline 118 & $2 s 2 p^{6} 4 f^{3} F^{0}$ & 2 & 952.848097 & 952.626 & $4.01 \mathrm{E}-15$ & 100 \\
\hline 119 & $2 s 2 p^{6} 4 f^{3} F^{0}$ & 3 & 952.831403 & 952.637 & $4.02 \mathrm{E}-15$ & $60.37+39.69(121)$ \\
\hline 120 & $2 s 2 p^{6} 4 f^{3} F^{0}$ & 4 & 953.910809 & 953.7059 & 4.11E-15 & 100 \\
\hline 121 & $2 s 2 p^{6} 4 f^{1} F^{\circ}$ & 4 & 953.993888 & 953.7822 & $4.13 \mathrm{E}-15$ & $60.37+39.69(119)$ \\
\hline 122 & $2 s^{2} 2 p^{5} 6 s^{3} p^{0}$ & 2 & 960.877736 & 960.9637 & $2.91 \mathrm{E}-14$ & 100 \\
\hline 123 & $2 s^{2} 2 p^{5} 6 s^{1} p^{0}$ & 1 & 960.926836 & 961.0092 & 2.07E-14 & $66.59+33.41(191)$ \\
\hline 124 & $2 s^{2} 2 p^{5} 6 p{ }^{3} p^{e}$ & 1 & 962.133913 & 962.2063 & $2.44 \mathrm{E}-14$ & $49.84+30.91(194)$ \\
\hline
\end{tabular}




\begin{tabular}{|c|c|c|c|c|c|c|}
\hline 125 & $2 s^{2} 2 p^{5} 6 p^{3} D^{e}$ & 2 & 962.143661 & 962.2299 & $2.42 \mathrm{E}-14$ & $49.98+33.41(195)+16.56(128)$ \\
\hline 126 & $2 s^{2} 2 p^{5} 6 p^{3} D^{e}$ & 3 & 965.028542 & 965.1508 & $3.41 \mathrm{E}-14$ & 100 \\
\hline 127 & $2 s^{2} 2 p^{5} 6 p^{1} p^{e}$ & 1 & 965.035272 & 965.1513 & 3.33E-14 & $56.4+35.76(194)$ \\
\hline 128 & $2 s^{2} 2 p^{5} 6 p^{3} p^{e}$ & 2 & 965.117698 & 965.2267 & $3.39 \mathrm{E}-14$ & $66.75+33.18(195)$ \\
\hline 129 & $2 s^{2} 2 p^{5} 6 p^{1} s^{e}$ & 0 & 965.463606 & 965.54 & 3.31E-14 & $66.42+33.52(193)$ \\
\hline 130 & $2 s^{2} 2 p^{5} 6 d^{3} p^{0}$ & 0 & 966.235633 & 966.3449 & $1.85 \mathrm{E}-14$ & 100 \\
\hline 131 & $2 s^{2} 2 p^{5} 6 d^{3} p^{0}$ & 1 & 966.301831 & 966.4302 & $1.61 \mathrm{E}-14$ & $63.2+34.34(197)$ \\
\hline 132 & $2 s^{2} 2 p^{5} 6 d^{3} F^{0}$ & 3 & 966.303614 & 966.4491 & $1.85 \mathrm{E}-14$ & $53.44+39.56(199)$ \\
\hline 133 & $2 s^{2} 2 p^{5} 6 d^{3} D^{0}$ & 2 & 966.357322 & 966.4835 & $1.84 \mathrm{E}-14$ & $54.46+18.23(135)+18.23(196)$ \\
\hline 134 & $2 s^{2} 2 p^{5} 6 d^{3} F^{0}$ & 4 & 967.006363 & 967.1704 & $1.85 \mathrm{E}-14$ & 100 \\
\hline 135 & $2 s^{2} 2 p^{5} 6 d^{1} D^{0}$ & 2 & 967.055448 & 967.2039 & $1.84 \mathrm{E}-14$ & $48.3+40.96(198)$ \\
\hline 136 & $2 s^{2} 2 p^{5} 6 d^{3} D^{0}$ & 3 & 967.122899 & 967.2694 & $1.84 \mathrm{E}-14$ & $70.9+27.04(199)$ \\
\hline 137 & $2 s^{2} 2 p^{5} 6 d^{1} p^{0}$ & 1 & 967.312534 & 967.4521 & $3.45 \mathrm{E}-15$ & $64+20.25(131)$ \\
\hline 138 & $2 s^{2} 2 p^{5} 7 s^{3} p^{0}$ & 2 & 993.005034 & 993.1369 & 3.97E-14 & 100 \\
\hline 139 & $2 s^{2} 2 p^{5} 7 s^{1} p^{0}$ & 1 & 993.035591 & 993.1647 & $3.02 \mathrm{E}-14$ & $66.59+33.41(201)$ \\
\hline 140 & $2 s^{2} 2 p^{5} 7 p^{3} p^{e}$ & 1 & 993.788223 & 993.9116 & 3.35E-14 & $49.98+30.91(204)$ \\
\hline 141 & $2 s^{2} 2 p^{5} 7 p^{3} D^{e}$ & 2 & 993.793913 & 993.9256 & 3.34E-14 & $49.98+33.41(205)+16.56(144)$ \\
\hline 142 & $2 s^{2} 2 p^{5} 7 p^{1} p^{e}$ & 1 & 995.599273 & 995.7497 & 4.34E-14 & $56.4+35.76(204)$ \\
\hline 143 & $2 s^{2} 2 p^{5} 7 p^{3} D^{e}$ & 3 & 995.595808 & 995.7501 & 4.54E-14 & 100 \\
\hline 144 & $2 s^{2} 2 p^{5} 7 p^{3} p^{e}$ & 2 & 995.651437 & 995.7969 & $4.48 \mathrm{E}-14$ & $66.75+33.18(205)$ \\
\hline 145 & $2 s^{2} 2 p^{5} 7 p^{1} s^{e}$ & 0 & 995.839882 & 995.9653 & 4.17E-14 & $66.26+33.41(203)$ \\
\hline 146 & $2 s^{2} 2 p^{5} 7 d^{3} p^{0}$ & 0 & 996.35125 & 996.4942 & $2.53 \mathrm{E}-14$ & 100 \\
\hline 147 & $2 s^{2} 2 p^{5} 7 d^{3} p^{0}$ & 1 & 996.395183 & 996.5447 & $2.53 \mathrm{E}-14$ & $63.2+34.34(207)$ \\
\hline 148 & $2 s^{2} 2 p^{5} 7 d^{3} F^{0}$ & 3 & 996.387333 & 996.5568 & $2.65 \mathrm{E}-14$ & $53.44+39.56(209)$ \\
\hline 149 & $2 s^{2} 2 p^{5} 7 d^{3} D^{0}$ & 2 & 996.420383 & 996.5777 & $2.64 \mathrm{E}-14$ & $54.46+18.23(151)+18.23(206)$ \\
\hline 150 & $2 s^{2} 2 p^{5} 7 d^{3} F^{0}$ & 4 & 996.830733 & 996.7036 & $2.71 \mathrm{E}-14$ & 100 \\
\hline 151 & $2 s^{2} 2 p^{5} 7 d^{1} D^{0}$ & 2 & 996.860384 & 996.7308 & $2.68 \mathrm{E}-14$ & $48.44+40.96(208)$ \\
\hline 152 & $2 s^{2} 2 p^{5} 7 d^{3} D^{0}$ & 3 & 996.902409 & 997.0121 & 2.67E-14 & $70.9+27.04(209)$ \\
\hline 153 & $2 s^{2} 2 p^{5} 7 d^{1} p^{0}$ & 1 & 997.020636 & 997.032 & $6.12 \mathrm{E}-15$ & $63.52+19.98(147)$ \\
\hline 154 & $2 s^{2} 2 p^{5} 5 s^{3} p^{0}$ & 0 & 996.497906 & 997.072 & $2.15 \mathrm{E}-14$ & 100 \\
\hline 155 & $2 s^{2} 2 p^{5} 5 s^{3} p^{0}$ & 1 & 996.521026 & 997.1836 & $1.53 \mathrm{E}-14$ & $66.1+33.06(67)$ \\
\hline 156 & $2 s^{2} 2 p^{5} 5 p^{3} D^{e}$ & 1 & 998.656767 & & 1.70E-14 & $73.96+22.28(73)$ \\
\hline 157 & $2 s^{2} 2 p^{5} 5 p^{3} p^{e}$ & 0 & 999.215302 & 999.3673 & $1.74 \mathrm{E}-14$ & $65.77+33.76(76)$ \\
\hline 158 & $2 s^{2} 2 p^{5} 5 p^{3} s^{e}$ & 1 & 1003.80039 & 1003.986 & $2.49 \mathrm{E}-14$ & $50.13+29.48(158)$ \\
\hline 159 & $2 s^{2} 2 p^{5} 5 p^{1} D^{e}$ & 2 & 1003.81383 & 1004 & $2.46 \mathrm{E}-14$ & $49.98+33.41(159)+16.65(74)$ \\
\hline 160 & $2 s^{2} 2 p^{5} 5 d^{3} F^{0}$ & 2 & 1006.00644 & 1006.185 & 1.25E-14 & $74.48+20.07(85)$ \\
\hline 161 & $2 s^{2} 2 p^{5} 5 d^{3} D^{0}$ & 1 & 1006.31733 & 1006.481 & $2.74 \mathrm{E}-15$ & $49.7+33.64(87)+16.48(79)$ \\
\hline 162 & $2 s^{2} 2 p^{5} 5 d^{3} p^{0}$ & 2 & 1007.30974 & 1007.49 & 1.23E-14 & $47.89+35.64(81)$ \\
\hline 163 & $2 s^{2} 2 p^{5} 5 d^{1} F^{0}$ & 3 & 1007.34548 & 1007.525 & $1.23 \mathrm{E}-14$ & $44.36+33.41(163)+22.28(86)$ \\
\hline 164 & $2 s^{2} 2 p^{5} 5 f^{3} G^{e}$ & 3 & 1008.54655 & 1008.734 & $8.24 \mathrm{E}-15$ & $73.44+19.1(94)$ \\
\hline 165 & $2 s^{2} 2 p^{5} 5 f^{3} F^{e}$ & 2 & 1008.58831 & 1008.775 & $8.08 \mathrm{E}-15$ & $44.49+33.29(93)+22.18(90)$ \\
\hline 166 & $2 s^{2} 2 p^{5} 5 f^{3} D^{e}$ & 3 & 1009.12652 & 1009.313 & $8.40 \mathrm{E}-15$ & $54.32+29.81(91)$ \\
\hline 167 & $2 s^{2} 2 p^{5} 5 f^{1} G^{e}$ & 4 & 1009.12643 & 1009.314 & $8.48 \mathrm{E}-15$ & $41.6+33.29(167)+25(95)$ \\
\hline 168 & $2 s^{2} 2 p^{5} 5 g^{3} H^{0}$ & 4 & 1009.29895 & 1009.476 & $1.46 \mathrm{E}-14$ & $72.42+18.49(104)$ \\
\hline 169 & $2 s^{2} 2 p^{5} 5 g{ }^{3} F^{0}$ & 3 & 1009.30672 & 1009.484 & $1.45 \mathrm{E}-14$ & $41.73+33.29(109)+25(169)$ \\
\hline 170 & $2 s^{2} 2 p^{5} 5 g{ }^{1} H^{0}$ & 5 & 1009.6381 & 1009.815 & $1.47 \mathrm{E}-14$ & $39.94+33.29(170)+26.73(105)$ \\
\hline 171 & $2 s^{2} 2 p^{5} 5 g^{3} F^{0}$ & 4 & 1009.64375 & 1009.821 & $1.47 \mathrm{E}-14$ & $57.61+26.63(99)$ \\
\hline 172 & $2 s 2 p^{6} 5 s^{3} s^{e}$ & 1 & 1030.90309 & 1030.747 & 1.47E-14 & 100 \\
\hline 173 & $2 s 2 p^{6} 5 s^{1} s^{e}$ & 0 & 1031.27821 & 1031.094 & $1.46 \mathrm{E}-14$ & 100 \\
\hline 174 & $2 s^{2} 2 p^{5} 5 d^{3} F^{\circ}$ & 0 & 1033.13273 & 1032.949 & $1.28 \mathrm{E}-14$ & 100 \\
\hline 175 & $2 s^{2} 2 p^{5} 5 d^{3} D^{0}$ & 1 & 1033.14713 & 1032.963 & $6.76 \mathrm{E}-15$ & $67.57+32.38(177)$ \\
\hline 176 & $2 s^{2} 2 p^{5} 5 d^{3} p^{0}$ & 2 & 1038.22163 & 1038.05 & $1.68 \mathrm{E}-14$ & 100 \\
\hline 177 & $2 s^{2} 2 p^{5} 5 d^{3} F^{\circ}$ & 1 & 1038.29202 & 1038.117 & $5.55 \mathrm{E}-15$ & $67.57+32.38(175)$ \\
\hline 178 & $2 s 2 p^{6} 5 d^{3} D^{e}$ & 1 & 1040.42399 & 1040.246 & $9.96 \mathrm{E}-15$ & 100 \\
\hline 179 & $2 s 2 p^{6} 5 d^{3} D^{e}$ & 2 & 1040.49232 & 1040.31 & $9.89 \mathrm{E}-15$ & $68.56+31.47(181)$ \\
\hline 180 & $2 s 2 p^{6} 5 d^{3} D^{e}$ & 3 & 1041.6795 & 1041.508 & $9.90 \mathrm{E}-15$ & 100 \\
\hline 181 & $2 s 2 p^{6} 5 d^{1} D^{e}$ & 2 & 1041.86173 & 1041.679 & $9.79 \mathrm{E}-15$ & $68.56+31.47(179)$ \\
\hline 182 & $2 s 2 p^{6} 5 f^{3} F^{0}$ & 2 & 1042.89009 & 1042.734 & $7.11 \mathrm{E}-15$ & 100 \\
\hline 183 & $2 s 2 p^{6} 5 f^{3} F^{0}$ & 3 & 1042.89078 & 1042.736 & $7.09 \mathrm{E}-15$ & $61.47+38.56(185)$ \\
\hline 184 & $2 s 2 p^{6} 5 f^{3} F^{0}$ & 4 & 1043.44049 & 1043.284 & $7.27 \mathrm{E}-15$ & 100 \\
\hline 185 & $2 s 2 p^{6} 5 f^{1} F^{0}$ & 3 & 1043.48686 & 1043.331 & $7.31 E-15$ & $61.47+38.56(183)$ \\
\hline 186 & $2 s 2 p^{6} 5 g{ }^{3} G^{e}$ & 3 & 1043.6219 & 1043.449 & 1.16E-14 & 100 \\
\hline 187 & $2 s 2 p^{6} 5 g{ }^{3} G^{e}$ & 4 & 1043.61044 & 1043.464 & 1.16E-14 & $55.8+44.09(189)$ \\
\hline 188 & $2 s 2 p^{6} 5 g^{3} G^{e}$ & 5 & 1043.94899 & 1043.788 & 1.17E-14 & 100 \\
\hline 189 & $2 s 2 p^{6} 5 g{ }^{1} G^{e}$ & 4 & 1043.96002 & 1043.802 & 1.17E-14 & $55.8+44.09(187)$ \\
\hline 190 & $2 s^{2} 2 p^{5} 6 s^{3} p^{0}$ & 0 & 1050.76549 & 1051.019 & $2.90 \mathrm{E}-14$ & 100 \\
\hline
\end{tabular}




\begin{tabular}{|c|c|c|c|c|c|c|}
\hline 191 & $2 s^{2} 2 p^{5} 6 s^{3} p^{0}$ & 1 & 1050.78136 & 1051.045 & $2.54 \mathrm{E}-14$ & $66.59+33.41(123)$ \\
\hline 192 & $2 s^{2} 2 p^{5} 6 p^{3} D^{e}$ & 1 & 1051.9925 & 1052.257 & $2.42 \mathrm{E}-14$ & $73.96+22.28(127)$ \\
\hline 193 & $2 s^{2} 2 p^{5} 6 p^{3} p^{e}$ & 0 & 1052.27448 & 1052.514 & $2.42 \mathrm{E}-14$ & $66.42+33.52(129)$ \\
\hline 194 & $2 s^{2} 2 p^{5} 6 p^{3} s^{e}$ & 1 & 1054.93577 & 1055.216 & $3.41 \mathrm{E}-14$ & $49.98+29.59(194)$ \\
\hline 195 & $2 s^{2} 2 p^{5} 6 p^{1} D^{e}$ & 2 & 1054.9409 & 1055.238 & $3.38 \mathrm{E}-14$ & $49.98+33.29(195)+16.65(128)$ \\
\hline 196 & $2 s^{2} 2 p^{5} 6 d^{3} F^{0}$ & 2 & 1056.17966 & 1056.497 & $1.85 \mathrm{E}-14$ & $74.65+19.98(135)$ \\
\hline 197 & $2 s^{2} 2 p^{5} 6 d^{3} D^{0}$ & 1 & 1056.347 & 1056.661 & $4.93 E-15$ & $49.84+33.52(137)+16.56(131)$ \\
\hline 198 & $2 s^{2} 2 p^{5} 6 d^{3} p^{0}$ & 2 & 1056.93562 & 1057.26 & $1.84 \mathrm{E}-14$ & $47.89+35.64(133)$ \\
\hline 199 & $2 s^{2} 2 p^{5} 6 d^{1} F^{0}$ & 3 & 1056.95418 & 1057.285 & $1.85 \mathrm{E}-14$ & $44.36+33.29(199)+22.28(136)$ \\
\hline 200 & $2 s^{2} 2 p^{5} 7 s^{3} p^{0}$ & 0 & 1082.89988 & 1083.199 & $3.99 \mathrm{E}-14$ & 100 \\
\hline 201 & $2 s^{2} 2 p^{5} 7 s^{3} p^{0}$ & 1 & 1082.90937 & 1083.217 & $3.58 \mathrm{E}-14$ & $66.59+33.41(139)$ \\
\hline 202 & $2 s^{2} 2 p^{5} 7 p{ }^{3} D^{e}$ & 1 & 1083.66454 & 1083.972 & 3.34E-14 & $74.13+22.28(142)$ \\
\hline 203 & $2 s^{2} 2 p^{5} 7 p{ }^{3} p^{e}$ & 0 & 1083.83715 & 1084.129 & 3.29E-14 & $66.59+33.52(145)$ \\
\hline 204 & $2 s^{2} 2 p^{5} 7 p{ }^{3} s^{e}$ & 1 & 1085.50216 & 1084.972 & $4.52 E-14$ & $49.98+29.59(204)$ \\
\hline 205 & $2 s^{2} 2 p^{5} 7 p^{1} D^{e}$ & 2 & 1085.5055 & 1085.168 & $4.50 \mathrm{E}-14$ & $49.98+33.29(205)+16.65(144)$ \\
\hline 206 & $2 s^{2} 2 p^{5} 7 d^{3} F^{0}$ & 2 & 1086.27443 & 1085.835 & $2.68 \mathrm{E}-14$ & $74.65+19.98(151)$ \\
\hline 207 & $2 s^{2} 2 p^{5} 7 d^{3} D^{0}$ & 1 & 1086.37728 & 1085.841 & $7.94 \mathrm{E}-15$ & $49.98+33.41(153)+16.65(147)$ \\
\hline 208 & $2 s^{2} 2 p^{5} 7 d^{3} p^{0}$ & 2 & 1086.75026 & 1086.25 & $2.69 \mathrm{E}-14$ & $47.89+35.64(149)$ \\
\hline 209 & $2 s^{2} 2 p^{5} 7 d^{1} F^{0}$ & 3 & 1086.76178 & 1086.259 & $2.70 \mathrm{E}-14$ & $44.36+33.29(209)+22.28(152)$ \\
\hline
\end{tabular}

Table 2: MCDF energies (in Ryd.), lifetimes (in s) and mixing coefficients in Ne-like-Ta

\begin{tabular}{|c|c|c|c|c|c|c|}
\hline S.No. & Label & J & $\begin{array}{l}\text { Level } \\
\text { (Ryd) }\end{array}$ & FAC & Lifetimes & Mixing coefficients \\
\hline 1 & $2 s^{2} 2 p^{61} s^{e}$ & 0 & 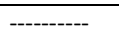 & 0 & -------- & 100 \\
\hline 2 & $2 s^{2} 2 p^{5} 3 s^{3} p^{0}$ & 2 & 592.5993 & 592.7375 & $2.63 \mathrm{E}-10$ & 100 \\
\hline 3 & $2 s^{2} 2 p^{5} 3 s^{1} p^{0}$ & 1 & 593.2078 & 593.3456 & $6.93 \mathrm{E}-15$ & $66.3+33.8(19)$ \\
\hline 4 & $2 s^{2} 2 p^{5} 3 p^{3} p^{e}$ & 1 & 603.4562 & 603.4645 & $1.67 \mathrm{E}-11$ & $49.4+31.6(25)$ \\
\hline 5 & $2 s^{2} 2 p^{5} 3 p^{3} D^{e}$ & 2 & 603.5888 & 603.5956 & $1.13 \mathrm{E}-12$ & $50.3+33.2(23)+16.5(8)$ \\
\hline 6 & $2 s^{2} 2 p^{5} 3 p^{1} p^{e}$ & 1 & 629.5819 & 629.6086 & 7.12E-13 & $56+35.9(25)$ \\
\hline 7 & $2 s^{2} 2 p^{5} 3 p^{3} D^{e}$ & 3 & 629.5512 & 629.635 & $6.79 \mathrm{E}-13$ & 100 \\
\hline 8 & $2 s^{2} 2 p^{5} 3 p^{3} p^{e}$ & 2 & 630.4977 & 630.5442 & 4.16E-13 & $66.7+33.3(23)$ \\
\hline 9 & $2 s^{2} 2 p^{5} 3 p^{1} s^{e}$ & 0 & 634.7089 & 634.6779 & 4.57E-13 & $61.9+37.9(21)$ \\
\hline 10 & $2 s^{2} 2 p^{5} 3 d^{3} p^{0}$ & 0 & 641.0699 & 641.0527 & $9.21 \mathrm{E}-13$ & 100 \\
\hline 11 & $2 s^{2} 2 p^{5} 3 d^{3} p^{0}$ & 1 & 641.79 & 641.7715 & $1.36 \mathrm{E}-14$ & $66.4+32(29)$ \\
\hline 12 & $2 s^{2} 2 p^{5} 3 d^{3} F^{0}$ & 3 & 641.8662 & 641.848 & $8.75 \mathrm{E}-13$ & $53.9+39.2(31)$ \\
\hline 13 & $2 s^{2} 2 p^{5} 3 d^{3} D^{0}$ & 2 & 642.4478 & 642.4236 & $8.33 \mathrm{E}-13$ & $54.5+18.2(28)+17.4(15)$ \\
\hline 14 & $2 s^{2} 2 p^{5} 3 d^{3} F^{0}$ & 4 & 647.5823 & 647.5906 & $6.84 \mathrm{E}-12$ & 100 \\
\hline 15 & $2 s^{2} 2 p^{5} 3 d^{1} D^{0}$ & 2 & 648.0979 & 648.0881 & 3.84E-12 & $48.6+41.1(30)$ \\
\hline 16 & $2 s^{2} 2 p^{5} 3 d^{3} D^{0}$ & 3 & 648.8295 & 648.8139 & $6.24 \mathrm{E}-12$ & $70.6+27.2(31)$ \\
\hline 17 & $2 s^{2} 2 p^{5} 3 d^{1} p^{0}$ & 1 & 650.9793 & 650.9179 & $3.80 \mathrm{E}-16$ & $62.9+19.1(29)+18(11)$ \\
\hline 18 & $2 s^{2} 2 p^{5} 3 s^{3} p^{0}$ & 0 & 688.2345 & 688.4583 & $3.78 \mathrm{E}-11$ & 99.8 \\
\hline 19 & $2 s^{2} 2 p^{5} 3 s^{3} p^{0}$ & 1 & 688.4805 & 688.7043 & $2.77 \mathrm{E}-14$ & $66.1+33.8(3)$ \\
\hline 20 & $2 s^{2} 2 p^{5} 3 p^{3} D^{e}$ & 1 & 698.8145 & 698.914 & $1.68 \mathrm{E}-11$ & $73.6+22.7(6)$ \\
\hline 21 & $2 s^{2} 2 p^{5} 3 p^{3} p^{e}$ & 0 & 702.3704 & 702.4376 & 3.27E-12 & $61.9+37.7(9)$ \\
\hline 22 & $2 s 2 p^{6} 3 s^{3} s^{e}$ & 1 & 722.7934 & 722.7092 & 7.79E-14 & 75.9 \\
\hline 23 & $2 s^{2} 2 p^{5} 3 p^{1} D^{e}$ & 2 & 725.508 & 725.6499 & $3.97 \mathrm{E}-13$ & $49.7+33.5(23)+16.8(1)$ \\
\hline 24 & $2 s 2 p^{6} 3 s^{1} s^{e}$ & 0 & 726.3644 & 725.9685 & $5.25 \mathrm{E}-14$ & 99.8 \\
\hline 25 & $2 s^{2} 2 p^{5} 3 p^{3} s^{e}$ & 1 & 726.4693 & 726.4161 & $1.01 \mathrm{E}-13$ & $37.8+24(22)+23(25)$ \\
\hline 26 & $2 s 2 p^{6} 3 p^{3} p^{0}$ & 0 & 734.8333 & 734.2853 & $4.94 \mathrm{E}-14$ & 99.8 \\
\hline 27 & $2 s 2 p^{6} 3 p^{3} p^{0}$ & 1 & 734.6042 & 734.4522 & $1.12 \mathrm{E}-15$ & $65.1+31.5(33)$ \\
\hline 28 & $2 s^{2} 2 p^{5} 3 d^{3} F^{0}$ & 2 & 737.4615 & 737.5445 & $8.37 \mathrm{E}-13$ & $74.1+20.4(15)$ \\
\hline 29 & $2 s^{2} 2 p^{5} 3 d^{3} D^{0}$ & 1 & 739.5 & 739.4816 & $8.84 \mathrm{E}-16$ & $47.1+34.3(12)$ \\
\hline 30 & $2 s^{2} 2 p^{5} 3 d^{3} p^{0}$ & 2 & 743.6703 & 743.7452 & $3.68 \mathrm{E}-12$ & $46.9+36.1(13)$ \\
\hline 31 & $2 s^{2} 2 p^{5} 3 d^{1} F^{0}$ & 3 & 743.9205 & 743.9977 & 5.67E-12 & $44+33.5(31)+22.5(16)$ \\
\hline 32 & $2 s 2 p^{6} 3 p^{3} p^{0}$ & 2 & 761.0678 & 760.7432 & 4.59E-14 & 99.6 \\
\hline 33 & $2 s 2 p^{6} 3 p^{1} p^{0}$ & 1 & 761.5023 & 761.1745 & $2.25 \mathrm{E}-15$ & $67.7+32.1(27)$ \\
\hline 34 & $2 s 2 p^{6} 3 d^{3} D^{e}$ & 1 & 773.0447 & 772.5914 & $5.12 \mathrm{E}-14$ & 100 \\
\hline 35 & $2 s 2 p^{6} 3 d^{3} D^{e}$ & 2 & 773.4308 & 772.9768 & $3.96 \mathrm{E}-14$ & $70.9+29.1(37)$ \\
\hline 36 & $2 s 2 p^{6} 3 d^{3} D^{e}$ & 3 & 779.0326 & 778.6028 & $5.07 \mathrm{E}-14$ & 100 \\
\hline
\end{tabular}




\begin{tabular}{|c|c|c|c|c|c|c|}
\hline 37 & $2 s 2 p^{6} 3 d^{1} D^{e}$ & 2 & 780.1552 & 779.7101 & $3.35 \mathrm{E}-14$ & $70.9+29.1(35)$ \\
\hline 38 & $2 s^{2} 2 p^{5} 4 s^{3} p^{0}$ & 2 & 829.1212 & 829.2306 & $1.56 \mathrm{E}-14$ & 100 \\
\hline 39 & $2 s^{2} 2 p^{5} 4 s^{1} p^{0}$ & 1 & 829.3256 & 829.4356 & $8.65 \mathrm{E}-15$ & $66.6+33.4(63)$ \\
\hline 40 & $2 s^{2} 2 p^{5} 4 p^{3} p^{e}$ & 1 & 833.6049 & 833.6663 & $1.29 \mathrm{E}-14$ & $49.7+31.2(70)$ \\
\hline 41 & $2 s^{2} 2 p^{5} 4 p^{3} D^{e}$ & 2 & 833.6431 & 833.7049 & $1.28 \mathrm{E}-14$ & $50.1+33.4(71)+16.5(44)$ \\
\hline 42 & $2 s^{2} 2 p^{5} 4 p^{3} D^{e}$ & 3 & 844.3212 & 844.402 & $1.99 \mathrm{E}-14$ & 100 \\
\hline 43 & $2 s^{2} 2 p^{5} 4 p^{1} p^{e}$ & 1 & 844.3446 & 844.424 & $1.98 \mathrm{E}-14$ & $56.4+35.8(70)$ \\
\hline 44 & $2 s^{2} 2 p^{5} 4 p^{3} p^{e}$ & 2 & 844.6705 & 844.7492 & $2.00 \mathrm{E}-14$ & $66.7+33.2(71)$ \\
\hline 45 & $2 s^{2} 2 p^{5} 4 p^{1} s^{e}$ & 0 & 846.1021 & 846.1192 & $2.07 \mathrm{E}-14$ & $65.4+34.5(65)$ \\
\hline 46 & $2 s^{2} 2 p^{5} 4 d^{3} p^{0}$ & 0 & 848.7364 & 848.7906 & $8.65 \mathrm{E}-15$ & 100 \\
\hline 47 & $2 s^{2} 2 p^{5} 4 d^{3} p^{0}$ & 1 & 848.9964 & 849.0485 & $6.93 \mathrm{E}-15$ & $64.3+33.5(85)$ \\
\hline 48 & $2 s^{2} 2 p^{5} 4 d^{3} F^{0}$ & 3 & 849.0097 & 849.0602 & $8.69 \mathrm{E}-15$ & $53.6+39.4(103)$ \\
\hline 49 & $2 s^{2} 2 p^{5} 4 d^{3} D^{0}$ & 2 & 849.2215 & 849.2681 & $8.73 \mathrm{E}-15$ & $54.5+18.2(80)+18(51)$ \\
\hline 50 & $2 s^{2} 2 p^{5} 4 d^{3} F^{0}$ & 4 & 851.5045 & 851.5643 & $8.38 \mathrm{E}-15$ & 100 \\
\hline 51 & $2 s^{2} 2 p^{5} 4 d^{1} D^{0}$ & 2 & 851.6954 & 851.7508 & $8.38 \mathrm{E}-15$ & $48 .+41(102)$ \\
\hline 52 & $2 s^{2} 2 p^{5} 4 d^{3} D^{0}$ & 3 & 851.958 & 852.0092 & $8.43 \mathrm{E}-15$ & $70.7+27.1(103)$ \\
\hline 53 & $2 s^{2} 2 p^{5} 4 d^{1} p^{0}$ & 1 & 852.711 & 852.7379 & $9.36 \mathrm{E}-16$ & $63.8+19.3(47)+16.8(85)$ \\
\hline 54 & $2 s^{2} 2 p^{5} 4 f^{3} D^{e}$ & 1 & 854.0658 & 854.1326 & $3.99 \mathrm{E}-15$ & 100 \\
\hline 55 & $2 s^{2} 2 p^{5} 4 f^{3} G^{e}$ & 4 & 854.0864 & 854.1534 & 4.09E-15 & $52.9+44.2(102)$ \\
\hline 56 & $2 s^{2} 2 p^{5} 4 f^{3} F^{e}$ & 2 & 854.2393 & 854.3062 & $4.03 E-15$ & $47.5+46.6(105)$ \\
\hline 57 & $2 s^{2} 2 p^{5} 4 f^{3} F^{e}$ & 3 & 854.3171 & 854.3839 & $4.08 \mathrm{E}-15$ & $50.7+22.8(104)+22.4(60)$ \\
\hline 58 & $2 s^{2} 2 p^{5} 4 f^{3} G^{e}$ & 5 & 855.2444 & 855.3115 & $4.19 \mathrm{E}-15$ & 100 \\
\hline 59 & $2 s^{2} 2 p^{5} 4 f^{1} D^{e}$ & 2 & 855.3683 & 855.4328 & $4.08 \mathrm{E}-15$ & $60.8+31.1(105)$ \\
\hline 60 & $2 s^{2} 2 p^{5} 4 f^{1} F^{e}$ & 3 & 855.4295 & 855.4958 & 4.16E-15 & $44.1+40.4(106)$ \\
\hline 61 & $2 s^{2} 2 p^{5} 4 f^{3} F^{e}$ & 4 & 855.4997 & 855.5665 & 4.20E-15 & $71.9+22.5(107)$ \\
\hline 62 & $2 s^{2} 2 p^{5} 4 s^{3} p^{0}$ & 0 & 924.8705 & 925.0771 & $1.54 \mathrm{E}-14$ & 100 \\
\hline 63 & $2 s^{2} 2 p^{5} 4 s^{3} p^{0}$ & 1 & 924.9393 & 925.1465 & $1.20 \mathrm{E}-14$ & $66.4+33.4(39)$ \\
\hline 64 & $2 s^{2} 2 p^{5} 4 p^{3} D^{e}$ & 1 & 929.2442 & 929.4027 & $1.27 \mathrm{E}-14$ & $74+22.4(43)$ \\
\hline 65 & $2 s^{2} 2 p^{5} 4 p^{3} p^{e}$ & 0 & 930.3735 & 930.505 & $1.31 \mathrm{E}-14$ & $65.4+34.2(45)$ \\
\hline 66 & $2 s^{2} 2 p^{5} 5 s^{3} p^{0}$ & 2 & 934.5911 & 934.7091 & $1.92 \mathrm{E}-14$ & 100 \\
\hline 67 & $2 s^{2} 2 p^{5} 5 s^{1} p^{0}$ & 1 & 934.6879 & 934.8045 & $1.20 \mathrm{E}-14$ & $66.6+33.4(155)$ \\
\hline 68 & $2 s^{2} 2 p^{5} 5 p^{3} p^{e}$ & 1 & 936.8377 & 936.9296 & $1.60 \mathrm{E}-14$ & $49.7+31.1(158)$ \\
\hline 69 & $2 s^{2} 2 p^{5} 5 p^{3} D^{e}$ & 2 & 936.8627 & 936.9541 & $1.59 \mathrm{E}-14$ & $50+33.4(151)+16.5(79)$ \\
\hline 70 & $2 s^{2} 2 p^{5} 4 p^{3} s^{e}$ & 1 & 940.1295 & 940.3073 & $2.00 \mathrm{E}-14$ & $49.3+28.7(70)$ \\
\hline 71 & $2 s^{2} 2 p^{5} 4 p^{1} D^{e}$ & 2 & 940.1822 & 940.3596 & $1.97 \mathrm{E}-14$ & $49.8+33.4(71)+16.7(49)$ \\
\hline 72 & $2 s^{2} 2 p^{5} 5 p^{3} D^{e}$ & 3 & 942.2441 & 942.3463 & $2.34 \mathrm{E}-14$ & 100 \\
\hline 73 & $2 s^{2} 2 p^{5} 5 p^{1} p^{e}$ & 1 & 942.2644 & 942.3644 & $2.29 \mathrm{E}-14$ & $55.2+35.3(158)$ \\
\hline 74 & $2 s^{2} 2 p^{5} 5 p^{3} p^{e}$ & 2 & 942.4154 & 942.514 & $2.35 \mathrm{E}-14$ & $66.7+33.2(159)$ \\
\hline 75 & $2 s^{2} 2 p^{5} 5 p^{1} s^{e}$ & 0 & 943.1085 & 943.1668 & $2.41 \mathrm{E}-14$ & $65.8+33.8(152)$ \\
\hline 76 & $2 s^{2} 2 p^{5} 5 d^{3} p^{0}$ & 0 & 944.414 & 944.5093 & $1.16 \mathrm{E}-14$ & 100 \\
\hline 77 & $2 s^{2} 2 p^{5} 5 d^{3} p^{0}$ & 1 & 944.5283 & 944.6241 & $7.80 \mathrm{E}-15$ & $64.6+33.3(161)$ \\
\hline 78 & $2 s^{2} 2 p^{5} 5 d^{3} F^{0}$ & 3 & 944.5392 & 944.6315 & $1.17 \mathrm{E}-14$ & $53.4+39.4(163)$ \\
\hline 79 & $2 s^{2} 2 p^{5} 5 d^{3} D^{0}$ & 2 & 944.7027 & 944.7024 & $1.03 \mathrm{E}-14$ & $54.2+18.1(160)+18(83)$ \\
\hline 80 & $2 s^{2} 2 p^{5} 4 d^{3} F^{0}$ & 2 & 944.6487 & 944.9086 & $9.71 \mathrm{E}-15$ & $60.1+17.1(51)$ \\
\hline 81 & $2 s^{2} 2 p^{5} 5 d^{3} F^{0}$ & 4 & 945.8335 & 945.3114 & $1.16 \mathrm{E}-14$ & 100 \\
\hline 82 & $2 s^{2} 2 p^{5} 5 d^{1} p^{0}$ & 1 & 945.1335 & 945.9316 & $3.01 \mathrm{E}-15$ & $32.8+25.5(85)+16.4(53)$ \\
\hline 83 & $2 s^{2} 2 p^{5} 5 d^{1} D^{0}$ & 2 & 945.9281 & 946.0059 & $1.14 \mathrm{E}-14$ & $41.1+32.4(162)$ \\
\hline 84 & $2 s^{2} 2 p^{5} 5 d^{3} D^{0}$ & 3 & 946.0513 & 946.1416 & $1.16 \mathrm{E}-14$ & $70.7+27.1(163)$ \\
\hline 85 & $2 s^{2} 2 p^{5} 4 d^{3} D^{0}$ & 1 & 946.6491 & 946.6734 & $1.11 \mathrm{E}-15$ & $31.5+24.1(85)+17.6(53)$ \\
\hline 86 & $2 s^{2} 2 p^{5} 5 f^{3} D^{e}$ & 1 & 947.0832 & 947.187 & 7.62E-15 & 100 \\
\hline 87 & $2 s^{2} 2 p^{5} 5 f^{3} G^{e}$ & 4 & 947.1071 & 947.2106 & $7.75 \mathrm{E}-15$ & $53+43.8(166)$ \\
\hline 88 & $2 s^{2} 2 p^{5} 5 f^{3} D^{e}$ & 2 & 947.1733 & 947.276 & $7.64 \mathrm{E}-15$ & $47.6+47.1(165)$ \\
\hline 89 & $2 s^{2} 2 p^{5} 5 f^{3} F^{e}$ & 3 & 947.2171 & 947.3188 & $7.71 \mathrm{E}-15$ & $50.8+22.7(164)+22.3(92)$ \\
\hline 90 & $2 s^{2} 2 p^{5} 5 f^{3} G^{e}$ & 5 & 947.6986 & 947.6019 & $7.96 \mathrm{E}-15$ & 100 \\
\hline 91 & $2 s^{2} 2 p^{5} 5 f^{1} D^{e}$ & 2 & 947.7756 & 947.6593 & $7.75 \mathrm{E}-15$ & $61.3+30.1(88)$ \\
\hline 92 & $2 s^{2} 2 p^{5} 5 f^{1} F^{e}$ & 3 & 947.7831 & 947.8026 & $7.89 \mathrm{E}-15$ & $44.2+40.3(166)$ \\
\hline 93 & $2 s^{2} 2 p^{5} 5 f^{3} F^{e}$ & 4 & 947.8279 & 947.8774 & $7.95 \mathrm{E}-15$ & $71.9+22.8(162)$ \\
\hline 94 & $2 s^{2} 2 p^{5} 5 g^{3} F^{0}$ & 2 & 947.8952 & 947.8857 & $1.37 \mathrm{E}-14$ & 100 \\
\hline 95 & $2 s^{2} 2 p^{5} 5 g^{3} H^{0}$ & 5 & 947.8998 & 947.9299 & $1.37 \mathrm{E}-14$ & $52.4+45.8(170)$ \\
\hline 96 & $2 s^{2} 2 p^{5} 5 g^{3} G^{0}$ & 3 & 947.9468 & 947.9882 & $1.37 \mathrm{E}-14$ & $54.2+37(169)$ \\
\hline 97 & $2 s^{2} 2 p^{5} 5 g^{3} G^{0}$ & 4 & 947.9654 & 947.9925 & 1.37E-14 & $47.5+25.2(168)+25(100)$ \\
\hline 98 & $2 s^{2} 2 p^{5} 5 g^{1} F^{0}$ & 3 & 948.2646 & 948.04 & $1.39 \mathrm{E}-14$ & $56.6+37(169)$ \\
\hline 99 & $2 s^{2} 2 p^{5} 5 g^{3} H^{0}$ & 6 & 948.2606 & 948.0585 & $1.38 \mathrm{E}-14$ & 100 \\
\hline 100 & $2 s^{2} 2 p^{5} 5 g^{1} G^{0}$ & 4 & 948.3096 & 948.3533 & $1.39 \mathrm{E}-14$ & $41.6+39.3(171)+17.5(97)$ \\
\hline 101 & $2 s^{2} 2 p^{5} 5 g^{3} G^{0}$ & 5 & 948.3248 & 948.3557 & $1.38 \mathrm{E}-14$ & $71.6+20.9(170)$ \\
\hline 102 & $2 s^{2} 2 p^{5} 4 d^{3} p^{0}$ & 2 & 947.4496 & 948.4028 & $8.40 \mathrm{E}-15$ & $47.3+35.6(49)$ \\
\hline 103 & $2 s^{2} 2 p^{5} 4 d^{1} F^{0}$ & 3 & 947.5041 & 948.418 & $8.43 \mathrm{E}-15$ & $43.2+32.6(103)+21.7(52)$ \\
\hline 104 & $2 s^{2} 2 p^{5} 4 f^{3} G^{e}$ & 3 & 949.9818 & 950.1468 & $4.09 \mathrm{E}-15$ & $73.3+19.1(60)$ \\
\hline 105 & $2 s^{2} 2 p^{5} 4 f^{3} D^{e}$ & 2 & 950.0504 & 950.215 & $4.02 \mathrm{E}-15$ & $44.6+33.3(59)+22.1(105)$ \\
\hline 106 & $2 s^{2} 2 p^{5} 4 f^{3} D^{e}$ & 3 & 951.1855 & 951.3421 & 4.17E-15 & $54.2+29.8(57)$ \\
\hline 107 & $2 s^{2} 2 p^{5} 4 f^{1} G^{e}$ & 4 & 951.1779 & 951.35 & 4.20E-15 & $41.6+33.3(107)+25.1(61)$ \\
\hline
\end{tabular}




\begin{tabular}{|c|c|c|c|c|c|c|}
\hline 108 & $2 s 2 p^{6} 4 s^{3} s^{e}$ & 1 & 960.1425 & 959.9373 & $1.18 \mathrm{E}-14$ & 99.8 \\
\hline 109 & $2 s 2 p^{6} 4 s^{1} s^{e}$ & 0 & 960.9429 & 960.6987 & 1.19E-14 & 100 \\
\hline 110 & $2 s 2 p^{6} 4 p^{3} p^{0}$ & 0 & 964.6587 & 964.403 & $1.03 \mathrm{E}-14$ & 100 \\
\hline 111 & $2 s 2 p^{6} 4 p^{3} p^{0}$ & 1 & 964.6892 & 964.4335 & 4.30E-15 & $67.6+32.4(113)$ \\
\hline 112 & $2 s 2 p^{6} 4 p^{3} p^{0}$ & 2 & 975.4349 & 975.2033 & $1.43 \mathrm{E}-14$ & 100 \\
\hline 113 & $2 s 2 p^{6} 4 p^{1} p^{0}$ & 1 & 975.5897 & 975.3549 & 3.39E-15 & $67.6+32.4(111)$ \\
\hline 114 & $2 s 2 p^{6} 4 d^{3} D^{e}$ & 1 & 980.0128 & 979.7429 & $7.39 \mathrm{E}-15$ & 100 \\
\hline 115 & $2 s 2 p^{6} 4 d^{3} D^{e}$ & 2 & 980.1481 & 979.8786 & $7.34 \mathrm{E}-15$ & $68.6+31.4(117)$ \\
\hline 116 & $2 s 2 p^{6} 4 d^{3} D^{e}$ & 3 & 982.6002 & 982.3453 & $7.16 \mathrm{E}-15$ & 100 \\
\hline 117 & $2 s 2 p^{6} 4 d^{1} D^{e}$ & 2 & 982.9937 & 982.7266 & $7.08 \mathrm{E}-15$ & $68.6+31.4(115)$ \\
\hline 118 & $2 s 2 p^{6} 4 f^{3} F^{0}$ & 2 & 985.1127 & 984.861 & $3.75 \mathrm{E}-15$ & 100 \\
\hline 119 & $2 s 2 p^{6} 4 f^{3} F^{0}$ & 3 & 985.0948 & 984.872 & $3.76 \mathrm{E}-15$ & $60.2+39.8(121)$ \\
\hline 120 & $2 s 2 p^{6} 4 f^{3} F^{0}$ & 4 & 986.2468 & 986.0134 & $3.85 \mathrm{E}-15$ & 100 \\
\hline 121 & $2 s 2 p^{6} 4 f^{1} F^{0}$ & 3 & 986.3313 & 986.0899 & $3.86 \mathrm{E}-15$ & $60.2+39.8(119)$ \\
\hline 122 & $2 s^{2} 2 p^{5} 6 s^{3} p^{0}$ & 2 & 990.6868 & 990.7681 & $2.72 \mathrm{E}-14$ & 100 \\
\hline 123 & $2 s^{2} 2 p^{5} 6 s^{1} p^{0}$ & 1 & 990.7364 & 990.814 & $1.94 \mathrm{E}-14$ & $66.6+33.4(191)$ \\
\hline 124 & $2 s^{2} 2 p^{5} 6 p^{3} p^{e}$ & 1 & 991.9697 & 992.0365 & $2.28 \mathrm{E}-14$ & $49.8+30.8(194)$ \\
\hline 125 & $2 s^{2} 2 p^{5} 6 p^{3} D^{e}$ & 2 & 991.9795 & 992.0607 & $2.27 \mathrm{E}-14$ & $50+33.4(195)+16.6(128)$ \\
\hline 126 & $2 s^{2} 2 p^{5} 6 p^{3} D^{e}$ & 3 & 995.059 & 995.1784 & $3.23 \mathrm{E}-14$ & 100 \\
\hline 127 & $2 s^{2} 2 p^{5} 6 p^{1} p^{e}$ & 1 & 995.0658 & 995.1787 & $3.15 \mathrm{E}-14$ & $56.4+35.9(194)$ \\
\hline 128 & $2 s^{2} 2 p^{5} 6 p^{3} p^{e}$ & 2 & 995.1499 & 995.2554 & $3.21 \mathrm{E}-14$ & $66.7+33.2(195)$ \\
\hline 129 & $2 s^{2} 2 p^{5} 6 p^{1} s^{e}$ & 0 & 995.5026 & 995.5745 & 3.13E-14 & $66.4+33.5(193)$ \\
\hline 130 & $2 s^{2} 2 p^{5} 6 d^{3} p^{0}$ & 0 & 996.287 & 996.3924 & $1.73 \mathrm{E}-14$ & 100 \\
\hline 131 & $2 s^{2} 2 p^{5} 6 d^{3} p^{0}$ & 1 & 996.3544 & 996.4799 & $1.51 \mathrm{E}-14$ & $62.9+34.6(197)$ \\
\hline 132 & $2 s^{2} 2 p^{5} 6 d^{3} F^{0}$ & 3 & 996.3551 & 996.4985 & $1.73 \mathrm{E}-14$ & $53.4+39.6(199)$ \\
\hline 133 & $2 s^{2} 2 p^{5} 6 d^{3} D^{0}$ & 2 & 996.4104 & 996.5336 & $1.73 \mathrm{E}-14$ & $54.3+18.3(133)+18.2(196)$ \\
\hline 134 & $2 s^{2} 2 p^{5} 6 d^{3} F^{0}$ & 4 & 997.1053 & 997.2682 & $1.74 \mathrm{E}-14$ & 100 \\
\hline 135 & $2 s^{2} 2 p^{5} 6 d^{1} D^{0}$ & 2 & 997.1557 & 997.3023 & $1.73 \mathrm{E}-14$ & $48.3+41(198)$ \\
\hline 136 & $2 s^{2} 2 p^{5} 6 d^{3} D^{0}$ & 3 & 997.2243 & 997.3689 & $1.73 \mathrm{E}-14$ & $70.9+27(199)$ \\
\hline 137 & $2 s^{2} 2 p^{5} 6 d^{1} p^{0}$ & 1 & 997.4167 & 997.5543 & $3.26 \mathrm{E}-15$ & $63.8+20.5(131)$ \\
\hline 138 & $2 s^{2} 2 p^{5} 7 s^{3} p^{0}$ & 2 & 1023.881 & 1024.01 & $3.71 \mathrm{E}-14$ & 100 \\
\hline 139 & $2 s^{2} 2 p^{5} 7 s^{1} p^{0}$ & 1 & 1023.912 & 1024.038 & $2.83 \mathrm{E}-14$ & $66.6+33.3(201)$ \\
\hline 140 & $2 s^{2} 2 p^{5} 7 p^{3} p^{e}$ & 1 & 1024.681 & 1024.801 & $3.13 \mathrm{E}-14$ & $50+30.9(204)$ \\
\hline 141 & $2 s^{2} 2 p^{5} 7 p^{3} D^{e}$ & 2 & 1024.686 & 1024.815 & $3.12 \mathrm{E}-14$ & $50+33.4(205)+16.6(144)$ \\
\hline 142 & $2 s^{2} 2 p^{5} 7 p^{3} D^{e}$ & 3 & 1026.609 & 1026.762 & 4. $29 \mathrm{E}-14$ & 100 \\
\hline 143 & $2 s^{2} 2 p^{5} 7 p^{1} p^{e}$ & 1 & 1026.613 & 1026.762 & $4.11 \mathrm{E}-14$ & $56.4+35.8(204)$ \\
\hline 144 & $2 s^{2} 2 p^{5} 7 p^{3} p^{e}$ & 2 & 1026.666 & 1026.81 & 4.23E-14 & $66.7+33.2(205)$ \\
\hline 145 & $2 s^{2} 2 p^{5} 7 p^{1} s^{e}$ & 0 & 1026.87 & 1026.991 & 3.97E-14 & $66.4+33.4(203)$ \\
\hline 146 & $2 s^{2} 2 p^{5} 7 d^{3} p^{0}$ & 0 & 1027.373 & 1027.519 & $2.51 \mathrm{E}-14$ & 100 \\
\hline 147 & $2 s^{2} 2 p^{5} 7 d^{3} p^{0}$ & 1 & 1027.414 & 1027.572 & $2.20 \mathrm{E}-14$ & $63+34.5(207)$ \\
\hline 148 & $2 s^{2} 2 p^{5} 7 d^{3} F^{0}$ & 3 & 1027.414 & 1027.582 & $2.50 \mathrm{E}-14$ & $53.4+39.6(209)$ \\
\hline 149 & $2 s^{2} 2 p^{5} 7 d^{3} D^{0}$ & 2 & 1027.448 & 1027.604 & $2.48 \mathrm{E}-14$ & $54.5+18.3(151)+18.2(206)$ \\
\hline 150 & $2 s^{2} 2 p^{5} 7 d^{3} F^{0}$ & 4 & 1027.888 & 1028.068 & $2.55 \mathrm{E}-14$ & 100 \\
\hline 151 & $2 s^{2} 2 p^{5} 7 d^{1} D^{0}$ & 2 & 1027.918 & 1028.089 & $2.52 \mathrm{E}-14$ & $48.3+41(208)$ \\
\hline 152 & $2 s^{2} 2 p^{5} 7 d^{3} D^{0}$ & 3 & 1027.961 & 1028.129 & $2.51 \mathrm{E}-14$ & $70.9+27(209)$ \\
\hline 153 & $2 s^{2} 2 p^{5} 7 d^{1} p^{0}$ & 1 & 1028.076 & 1028.238 & $5.49 \mathrm{E}-15$ & $64+20.3(147)$ \\
\hline 154 & $2 s^{2} 2 p^{5} 5 s^{3} p^{0}$ & 0 & 1030.389 & 1028.495 & $1.92 \mathrm{E}-14$ & 100 \\
\hline 155 & $2 s^{2} 2 p^{5} 5 s^{3} p^{0}$ & 1 & 1030.421 & 1028.518 & $1.68 \mathrm{E}-14$ & $66.6+33.4(67)$ \\
\hline 156 & $2 s^{2} 2 p^{5} 5 p^{3} D^{e}$ & 1 & 1032.588 & 1032.776 & $1.59 \mathrm{E}-14$ & $74+22.3(73)$ \\
\hline 157 & $2 s^{2} 2 p^{5} 5 p^{3} p^{e}$ & 0 & 1033.148 & 1033.312 & $1.62 \mathrm{E}-14$ & $66.1+33.8(75)$ \\
\hline 158 & $2 s^{2} 2 p^{5} 5 p^{3} s^{e}$ & 1 & 1038.076 & 1038.272 & $2.35 \mathrm{E}-14$ & $50.1+29.5(158)$ \\
\hline 159 & $2 s^{2} 2 p^{5} 5 p^{1} D^{e}$ & 2 & 1038.089 & 1038.286 & $2.33 \mathrm{E}-14$ & $50+33.4(151)+16.6(74)$ \\
\hline 160 & $2 s^{2} 2 p^{5} 5 d^{3} F^{0}$ & 2 & 1040.319 & 1040.508 & 1.17E-14 & $74.6+20.1(83)$ \\
\hline 161 & $2 s^{2} 2 p^{5} 5 d^{3} D^{0}$ & 1 & 1040.633 & 1040.808 & $2.59 \mathrm{E}-15$ & $49.8+33.6(82)+16.5(77)$ \\
\hline 162 & $2 s^{2} 2 p^{5} 5 d^{3} p^{0}$ & 2 & 1041.707 & 1041.898 & $1.15 \mathrm{E}-14$ & $47.9+35.6(79)$ \\
\hline 163 & $2 s^{2} 2 p^{5} 5 d^{1} F^{\circ}$ & 3 & 1041.743 & 1041.933 & $1.16 \mathrm{E}-14$ & $44.4+33.4(163)+22.3(84)$ \\
\hline 164 & $2 s^{2} 2 p^{5} 5 f^{3} G^{e}$ & 3 & 1042.963 & 1043.161 & $7.73 \mathrm{E}-15$ & $73.4+19.1(92)$ \\
\hline 165 & $2 s^{2} 2 p^{5} 5 f^{3} F^{e}$ & 2 & 1043.005 & 1043.203 & $7.58 \mathrm{E}-15$ & $44.5+33.3(91)+22.2(88)$ \\
\hline 166 & $2 s^{2} 2 p^{5} 5 f^{3} D^{e}$ & 3 & 1043.58 & 1043.778 & $7.90 \mathrm{E}-15$ & $54.3+29.8(89)$ \\
\hline 167 & $2 s^{2} 2 p^{5} 5 f^{1} G^{e}$ & 4 & 1043.58 & 1043.779 & 7.97E-15 & $41.6+33.3(167)+25(93)$ \\
\hline 168 & $2 s^{2} 2 p^{5} 5 g^{3} H^{0}$ & 4 & 1043.755 & 1043.943 & 1.37E-14 & $72.4+18.5(100)$ \\
\hline 169 & $2 s^{2} 2 p^{5} 5 g^{3} F^{\circ}$ & 3 & 1043.763 & 1043.952 & $1.36 \mathrm{E}-14$ & $41.7+33.3(98)+25(169)$ \\
\hline 170 & $2 s^{2} 2 p^{5} 5 g^{1} H^{\circ}$ & 5 & 1044.117 & 1044.305 & $1.38 \mathrm{E}-14$ & $39.9+33.3(170)+26.7(101)$ \\
\hline 171 & $2 s^{2} 2 p^{5} 5 g^{3} F^{0}$ & 4 & 1044.122 & 1044.311 & $1.38 \mathrm{E}-14$ & $57.6+26.6(97)$ \\
\hline 172 & $2 s 2 p^{6} 5 s^{3} s^{e}$ & 1 & 1065.542 & 1065.362 & $1.35 \mathrm{E}-14$ & 100 \\
\hline 173 & $2 s 2 p^{6} 5 s^{1} s^{e}$ & 0 & 1065.925 & 1065.717 & $1.34 \mathrm{E}-14$ & 100 \\
\hline 174 & $2 s 2 p^{6} 5 p^{3} p^{0}$ & 0 & 1067.82 & 1067.61 & $1.18 \mathrm{E}-14$ & 100 \\
\hline 175 & $2 s 2 p^{6} 5 p^{3} p^{0}$ & 1 & 1067.834 & 1067.623 & $6.28 \mathrm{E}-15$ & $67.6+32.4(177)$ \\
\hline 176 & $2 s 2 p^{6} 5 p^{3} p^{0}$ & 2 & 1073.25 & 1073.053 & $1.55 \mathrm{E}-14$ & 100 \\
\hline 177 & $2 s 2 p^{6} 5 p^{1} p^{0}$ & 1 & 1073.322 & 1073.121 & $5.22 \mathrm{E}-15$ & $67.6+32.4(175)$ \\
\hline 178 & $2 s 2 p^{6} 5 d^{3} D^{e}$ & 1 & 1075.491 & 1075.286 & $9.26 \mathrm{E}-15$ & 100 \\
\hline
\end{tabular}




\begin{tabular}{|c|c|c|c|c|c|c|}
\hline 179 & $2 s 2 p^{6} 5 d^{3} D^{e}$ & 2 & 1075.56 & 1075.351 & $9.19 \mathrm{E}-15$ & $68.2+31.8(181)$ \\
\hline 180 & $2 s 2 p^{6} 5 d^{3} D^{e}$ & 3 & 1076.828 & 1076.63 & $9.20 \mathrm{E}-15$ & 100 \\
\hline 181 & $2 s 2 p^{6} 5 d^{1} D^{e}$ & 2 & 1077.013 & 1076.804 & $9.10 \mathrm{E}-15$ & $68.2+31.8(179)$ \\
\hline 182 & $2 s 2 p^{6} 5 f^{3} F^{0}$ & 2 & 1078.058 & 1077.875 & $6.62 \mathrm{E}-15$ & 100 \\
\hline 183 & $2 s 2 p^{6} 5 f^{3} F^{0}$ & 3 & 1078.058 & 1077.877 & $6.61 \mathrm{E}-15$ & $61.3+38.7(185)$ \\
\hline 184 & $2 s 2 p^{6} 5 f^{3} F^{0}$ & 4 & 1078.645 & 1078.463 & $6.78 \mathrm{E}-15$ & 100 \\
\hline 185 & $2 s 2 p^{6} 5 f^{1} F^{0}$ & 3 & 1078.693 & 1078.511 & $6.81 \mathrm{E}-15$ & $61.3+38.7(183)$ \\
\hline 186 & $2 s 2 p^{6} 5 g^{3} G^{e}$ & 3 & 1078.83 & 1078.631 & $1.08 \mathrm{E}-14$ & 100 \\
\hline 187 & $2 s 2 p^{6} 5 g^{3} G^{e}$ & 4 & 1078.818 & 1078.646 & $1.07 \mathrm{E}-14$ & $55.8+44.2(189)$ \\
\hline 188 & $2 s 2 p^{6} 5 g^{3} G^{e}$ & 5 & 1079.179 & 1078.991 & $1.08 \mathrm{E}-14$ & 100 \\
\hline 189 & $2 s 2 p^{6} 5 g^{1} G^{e}$ & 4 & 1079.19 & 1079.006 & $1.09 \mathrm{E}-14$ & $55.8+44.2(187)$ \\
\hline 190 & $2 s^{2} 2 p^{5} 6 s^{3} p^{0}$ & 0 & 1086.461 & 1086.721 & 2.70E-14 & 100 \\
\hline 191 & $2 s^{2} 2 p^{5} 6 s^{3} p^{0}$ & 1 & 1086.477 & 1086.747 & $2.38 \mathrm{E}-14$ & $66.6+33.4(123)$ \\
\hline 192 & $2 s^{2} 2 p^{5} 6 p^{3} D^{e}$ & 1 & 1087.713 & 1087.984 & $2.26 \mathrm{E}-14$ & $74+22.3(127)$ \\
\hline 193 & $2 s^{2} 2 p^{5} 6 p^{3} p^{e}$ & 0 & 1088.002 & 1088.247 & $2.26 \mathrm{E}-14$ & $66.4+33.5(129)$ \\
\hline 194 & $2 s^{2} 2 p^{5} 6 p^{3} s^{e}$ & 1 & 1090.853 & 1091.141 & 3.22E-14 & $50+29.6(194)$ \\
\hline 195 & $2 s^{2} 2 p^{5} 6 p^{1} D^{e}$ & 2 & 1090.858 & 1091.163 & $3.19 \mathrm{E}-14$ & $50+33.3(195)+16.6(128)$ \\
\hline 196 & $2 s^{2} 2 p^{5} 6 d^{3} F^{0}$ & 2 & 1092.118 & 1092.444 & $1.74 \mathrm{E}-14$ & $74.6+20(135)$ \\
\hline 197 & $2 s^{2} 2 p^{5} 6 d^{3} D^{0}$ & 1 & 1092.288 & 1092.611 & 4.63E-15 & $49.8+33.5(137)+16.6(131)$ \\
\hline 198 & $2 s^{2} 2 p^{5} 6 d^{3} p^{0}$ & 2 & 1092.923 & 1093.256 & $1.73 \mathrm{E}-14$ & $47.9+35.6(133)$ \\
\hline 199 & $2 s^{2} 2 p^{5} 6 d^{1} F^{0}$ & 3 & 1092.942 & 1093.282 & $1.74 \mathrm{E}-14$ & $44.4+33.3(199)+22.3(136)$ \\
\hline 200 & $2 s^{2} 2 p^{5} 7 s^{3} p^{0}$ & 0 & 1119.663 & 1119.969 & $3.73 \mathrm{E}-14$ & 100 \\
\hline 201 & $2 s^{2} 2 p^{5} 7 s^{3} p^{0}$ & 1 & 1119.672 & 1119.988 & $3.35 \mathrm{E}-14$ & $66.6+33.4(139)$ \\
\hline 202 & $2 s^{2} 2 p^{5} 7 p^{3} D^{e}$ & 1 & 1120.443 & 1120.758 & $3.13 \mathrm{E}-14$ & $74.1+22.3(143)$ \\
\hline 203 & $2 s^{2} 2 p^{5} 7 p^{3} p^{e}$ & 0 & 1120.62 & 1120.919 & $3.08 \mathrm{E}-14$ & $66.6+33.5(145)$ \\
\hline 204 & $2 s^{2} 2 p^{5} 7 p^{3} s^{e}$ & 1 & 1122.404 & 1121.396 & $4.28 \mathrm{E}-14$ & $50+29.6(204)$ \\
\hline 205 & $2 s^{2} 2 p^{5} 7 p^{1} D^{e}$ & 2 & 1122.407 & 1121.59 & 4.26E-14 & $50+33.3(205)+16.6(144)$ \\
\hline 206 & $2 s^{2} 2 p^{5} 7 d^{3} F^{0}$ & 2 & 1123.189 & 1122.694 & 2.52E-14 & $74.6+20(151)$ \\
\hline 207 & $2 s^{2} 2 p^{5} 7 d^{3} D^{0}$ & 1 & 1123.293 & 1122.707 & $7.46 \mathrm{E}-15$ & $50+33.4(153)+16.6(147)$ \\
\hline 208 & $2 s^{2} 2 p^{5} 7 d^{3} p^{0}$ & 2 & 1123.695 & 1122.745 & 2.53E-14 & $47.9+35.6(149)$ \\
\hline 209 & $2 s^{2} 2 p^{5} 7 d^{1} F^{0}$ & 3 & 1123.707 & 1122.745 & $2.54 \mathrm{E}-14$ & $44.4+33.3(209)+22.3(152)$ \\
\hline
\end{tabular}

Table 3: MCDF energies (in Ryd.), lifetimes (in s) and mixing coefficients in Ne-like-W

\begin{tabular}{|c|c|c|c|c|c|c|c|c|}
\hline S.No & Label & J & $\begin{array}{l}\text { Level } \\
\text { (Ryd) }\end{array}$ & FAC & NIST & Ref. [24] & Lifetimes & Mixing coefficients \\
\hline 1 & $2 s^{2} 2 p^{6}{ }^{1} s^{e}$ & 0 & --------- & 0 & 0 & 0.0000 & .--.--.-- & 100 \\
\hline 2 & $2 s^{2} 2 p^{5} 3 s^{3} p^{0}$ & 2 & 609.646919 & 609.7976 & & 609.6024 & $2.31 \mathrm{E}-10$ & 100 \\
\hline 3 & $2 s^{2} 2 p^{5} 3 s^{1} p^{0}$ & 1 & 610.267422 & 610.4177 & 610.6401 & 610.2292 & $6.48 \mathrm{E}-15$ & $66.26+33.76(19)$ \\
\hline 4 & $2 s^{2} 2 p^{5} 3 p^{3} p^{e}$ & 1 & 620.748586 & 620.7565 & & 620.6689 & $1.53 \mathrm{E}-11$ & $49.42+31.58(25)$ \\
\hline 5 & $2 s^{2} 2 p^{5} 3 p^{3} D^{e}$ & 2 & 620.877261 & 620.8838 & & 620.7976 & $1.04 \mathrm{E}-12$ & $50.27+33.29(24)+16.48(8)$ \\
\hline 6 & $2 s^{2} 2 p^{5} 3 p^{1} p^{e}$ & 1 & 648.608985 & 648.636 & & 648.5388 & $6.28 \mathrm{E}-13$ & $55.95+36(25)$ \\
\hline 7 & $2 s^{2} 2 p^{5} 3 p^{3} D^{e}$ & 3 & 648.575977 & 648.6648 & & 648.5082 & $6.00 \mathrm{E}-13$ & 100 \\
\hline 8 & $2 s^{2} 2 p^{5} 3 p^{3} p^{e}$ & 2 & 649.54187 & 649.591 & & 649.4658 & $3.72 \mathrm{E}-13$ & $66.75+33.29(24)$ \\
\hline 9 & $2 s^{2} 2 p^{5} 3 p^{1} s^{e}$ & 0 & 653.837013 & 653.808 & 653.8587 & 653.7288 & $4.08 \mathrm{E}-13$ & $62.25+37.7(21)$ \\
\hline 10 & $2 s^{2} 2 p^{5} 3 d^{3} p^{0}$ & 0 & 660.298438 & 660.2802 & & 660.2404 & $8.19 E-13$ & 100 \\
\hline 11 & $2 s^{2} 2 p^{5} 3 d^{3} p^{0}$ & 1 & 661.03207 & 661.0127 & 661.507 & 660.9754 & $1.21 \mathrm{E}-14$ & $66.1+23.26(29)$ \\
\hline 12 & $2 s^{2} 2 p^{5} 3 d^{3} F^{0}$ & 3 & 661.09547 & 661.0764 & & 661.0420 & $7.79 \mathrm{E}-13$ & $53.88+39.19(31)$ \\
\hline 13 & $2 s^{2} 2 p^{5} 3 d^{3} D^{0}$ & 2 & 661.693976 & 661.6689 & & 661.6386 & $7.43 \mathrm{E}-13$ & $54.46+18.32(28)+17.56(15)$ \\
\hline 14 & $2 s^{2} 2 p^{5} 3 d^{3} F^{0}$ & 4 & 667.199226 & 667.2074 & & 667.1448 & $6.41 \mathrm{E}-12$ & 100 \\
\hline 15 & $2 s^{2} 2 p^{5} 3 d^{1} D^{0}$ & 2 & 667.7274 & 667.7173 & & 667.6732 & $3.52 \mathrm{E}-12$ & $48.44+41.09(30)$ \\
\hline 16 & $2 s^{2} 2 p^{5} 3 d^{3} D^{0}$ & 3 & 668.470303 & 668.4545 & & 668.4160 & $5.85 \mathrm{E}-12$ & $70.56+27.25(31)$ \\
\hline 17 & $2 s^{2} 2 p^{5} 3 d^{1} p^{0}$ & 1 & 670.649232 & 670.5868 & 670.246 & 670.5722 & $3.59 \mathrm{E}-16$ & $62.88+18.75(29)+18.23(11)$ \\
\hline 18 & $2 s^{2} 2 p^{5} 3 s^{3} p^{0}$ & 0 & 711.476523 & 711.721 & & 711.4577 & $3.16 \mathrm{E}-11$ & 99.8 \\
\hline 19 & $2 s^{2} 2 p^{5} 3 s^{3} p^{0}$ & 1 & 711.723347 & 711.9678 & 711.936 & 711.7088 & $2.56 \mathrm{E}-14$ & $66.26+33.76(3)$ \\
\hline 20 & $2 s^{2} 2 p^{5} 3 p^{3} D^{e}$ & 1 & 722.283258 & 722.3909 & & 722.2374 & $1.52 \mathrm{E}-11$ & $73.62+22.66(6)$ \\
\hline 21 & $2 s^{2} 2 p^{5} 3 p^{3} p^{e}$ & 0 & 725.916062 & 725.9919 & 726.088 & 725.8494 & $3.01 \mathrm{E}-12$ & $62.25+37.58(9)$ \\
\hline 22 & $2 s 2 p^{6} 3 s^{3} s^{e}$ & 1 & 747.074015 & 746.9027 & & 746.8320 & $5.64 \mathrm{E}-14$ & 85.01 \\
\hline 23 & $2 s 2 p^{6} 3 s^{1} s^{e}$ & 0 & 750.437584 & 750.0166 & & 750.0804 & $4.64 \mathrm{E}-14$ & 99.8 \\
\hline 24 & $2 s^{2} 2 p^{5} 3 p^{1} D^{e}$ & 2 & 750.730625 & 750.8837 & & 750.6897 & $3.55 \mathrm{E}-13$ & $49.7+33.52(24)+16.81(8)$ \\
\hline 25 & $2 s^{2} 2 p^{5} 3 p^{3} s^{e}$ & 1 & 751.427697 & 751.4487 & & 751.3483 & $1.27 \mathrm{E}-13$ & $42.51+25.4(25)$ \\
\hline 26 & $2 s 2 p^{6} 3 p^{3} p^{0}$ & 0 & 759.093117 & 758.5398 & & 758.8086 & 4.37E-14 & 99.8 \\
\hline 27 & $2 s 2 p^{6} 3 p^{3} p^{0}$ & 1 & 758.914026 & 758.6759 & 758.302 & 758.6382 & $1.22 \mathrm{E}-15$ & $65.93+31.81(33)$ \\
\hline 28 & $2 s^{2} 2 p^{5} 3 d^{3} F^{0}$ & 2 & 762.889496 & 762.98 & & 762.8610 & 7.44E-13 & $74.3+20.43(15)$ \\
\hline 29 & $2 s^{2} 2 p^{5} 3 d^{3} D^{0}$ & 1 & 764.890545 & 764.8985 & 765.027 & 764.8414 & $7.53 \mathrm{E}-16$ & $47.61+34.57(17)$ \\
\hline 30 & $2 s^{2} 2 p^{5} 3 d^{3} p^{0}$ & 2 & 769.496164 & 769.5792 & & 769.4660 & 3.36E-12 & $46.92+36.12(13)$ \\
\hline
\end{tabular}




\begin{tabular}{|c|c|c|c|c|c|c|c|c|}
\hline 31 & $2 s^{2} 2 p^{5} 3 d^{1} F^{0}$ & 3 & 769.747452 & 769.8329 & & 769.7196 & $5.23 \mathrm{E}-12$ & $43.96+33.52(31)+22.47(16)$ \\
\hline 32 & $2 s 2 p^{6} 3 p^{3} p^{0}$ & 2 & 787.066016 & 786.7087 & & 786.8023 & $4.06 \mathrm{E}-14$ & 99.6 \\
\hline 33 & $2 s 2 p^{6} 3 p{ }^{1} p^{0}$ & 1 & 787.508545 & 787.1479 & 786.651 & 787.2457 & $2.13 E-15$ & $67.73+32.15(27)$ \\
\hline 34 & $2 s 2 p^{6} 3 d^{3} D^{e}$ & 1 & 799.249757 & 798.7594 & & 798.9331 & $4.53 \mathrm{E}-14$ & 100 \\
\hline 35 & $2 s 2 p^{6} 3 d^{3} D^{e}$ & 2 & 799.639421 & 799.1486 & & 799.3305 & 3.51E-14 & $70.39+29.48(37)$ \\
\hline 36 & $2 s 2 p^{6} 3 d^{3} D^{e}$ & 3 & 805.624622 & 805.1589 & & 805.3202 & 4.49E-14 & 100 \\
\hline 37 & $2 s 2 p^{6} 3 d^{1} D^{e}$ & 2 & 806.760841 & 806.2799 & & 806.4689 & $3.00 \mathrm{E}-14$ & $70.39+29.48(35)$ \\
\hline 38 & $2 s^{2} 2 p^{5} 4 s^{3} p^{0}$ & 2 & 854.054641 & 854.1707 & & 853.9625 & $1.45 \mathrm{E}-14$ & 100 \\
\hline 39 & $2 s^{2} 2 p^{5} 4 s^{1} p^{0}$ & 1 & 854.262836 & 854.3794 & & 854.1708 & $8.08 \mathrm{E}-15$ & $66.59+33.41(63)$ \\
\hline 40 & $2 s^{2} 2 p^{5} 4 p^{3} p^{e}$ & 1 & 858.637158 & 858.7001 & & 858.5362 & $1.20 \mathrm{E}-14$ & $49.7+31.14(74)$ \\
\hline 41 & $2 s^{2} 2 p^{5} 4 p^{3} D^{e}$ & 2 & 858.674115 & 858.7374 & & 858.5732 & 1.19E-14 & $50.13+33.41(75)+16.48(44)$ \\
\hline 42 & $2 s^{2} 2 p^{5} 4 p^{3} D^{e}$ & 3 & 870.066369 & 870.15 & & 869.9708 & $1.89 \mathrm{E}-14$ & 100 \\
\hline 43 & $2 s^{2} 2 p^{5} 4 p^{1} p^{e}$ & 1 & 870.090721 & 870.173 & & 869.9943 & $1.88 \mathrm{E}-14$ & $56.25+35.88(74)$ \\
\hline 44 & $2 s^{2} 2 p^{5} 4 p^{3} p^{e}$ & 2 & 870.423072 & 870.5046 & & 870.3249 & $1.90 \mathrm{E}-14$ & $66.75+33.18(75)$ \\
\hline 45 & $2 s^{2} 2 p^{5} 4 p^{1} s^{e}$ & 0 & 871.881374 & 871.9004 & & 871.7391 & $1.96 \mathrm{E}-14$ & $65.45+34.46(65)$ \\
\hline 46 & $2 s^{2} 2 p^{5} 4 d^{3} p^{0}$ & 0 & 874.558949 & 874.6141 & & 874.4582 & 8.15E-15 & 100 \\
\hline 47 & $2 s^{2} 2 p^{5} 4 d^{3} p^{0}$ & 1 & 874.823461 & 874.8765 & & 874.7215 & $6.46 \mathrm{E}-15$ & $64+33.76(101)$ \\
\hline 48 & $2 s^{2} 2 p^{5} 4 d^{3} F^{0}$ & 3 & 874.832399 & 874.884 & & 874.7301 & $8.18 \mathrm{E}-15$ & $53.58+39.44(103)$ \\
\hline 49 & $2 s^{2} 2 p^{5} 4 d^{3} D^{0}$ & 2 & 875.050366 & 875.098 & & 874.9459 & $8.22 \mathrm{E}-15$ & $54.46+18.23(100)+18.06(51)$ \\
\hline 50 & $2 s^{2} 2 p^{5} 4 d^{3} F^{0}$ & 4 & 877.494508 & 877.5557 & & 877.3960 & $7.88 \mathrm{E}-15$ & 100 \\
\hline 51 & $2 s^{2} 2 p^{5} 4 d^{1} D^{0}$ & 2 & 877.690124 & 877.7469 & & 877.5893 & $7.88 \mathrm{E}-15$ & $48.44+40.96(102)$ \\
\hline 52 & $2 s^{2} 2 p^{5} 4 d^{3} D^{0}$ & 3 & 877.956988 & 878.0095 & & 877.8536 & $7.93 \mathrm{E}-15$ & $70.73+27.04(103)$ \\
\hline 53 & $2 s^{2} 2 p^{5} 4 d^{1} p^{0}$ & 1 & 878.719929 & 878.7479 & & 878.6101 & 8.83E-16 & $63.68+19.62(47)+16.56(101)$ \\
\hline 54 & $2 s^{2} 2 p^{5} 4 f^{3} D^{e}$ & 1 & 880.095934 & 880.1641 & & 880.0130 & $3.75 \mathrm{E}-15$ & 100 \\
\hline 55 & $2 s^{2} 2 p^{5} 4 f^{3} G^{e}$ & 4 & 880.113961 & 880.1824 & & 880.0340 & $3.84 \mathrm{E}-15$ & $52.85+44.09(107)$ \\
\hline 56 & $2 s^{2} 2 p^{5} 4 f^{3} F^{e}$ & 2 & 880.271846 & 880.34 & & & $3.79 \mathrm{E}-15$ & $47.75+46.38(105)$ \\
\hline 57 & $2 s^{2} 2 p^{5} 4 f^{3} F^{e}$ & 3 & 880.349935 & 880.4182 & & 880.2681 & $3.84 \mathrm{E}-15$ & $50.55+22.75(104)+22.47(60)$ \\
\hline 58 & $2 s^{2} 2 p^{5} 4 f^{3} G^{e}$ & 5 & 881.348167 & 881.4167 & & 881.2677 & $3.94 \mathrm{E}-15$ & 100 \\
\hline 59 & $2 s^{2} 2 p^{5} 4 f^{1} D^{e}$ & 2 & 881.476083 & 881.5419 & & 881.3954 & 3.83E-15 & $60.68+31.47(105)$ \\
\hline 60 & $2 s^{2} 2 p^{5} 4 f^{1} F^{e}$ & 3 & 881.53782 & 881.6055 & & 881.4557 & $3.91 \mathrm{E}-15$ & $44.09+40.45(106)$ \\
\hline 61 & $2 s^{2} 2 p^{5} 4 f^{3} F^{e}$ & 4 & 881.608563 & 881.6768 & & 881.5273 & $3.95 \mathrm{E}-15$ & $71.91+22.56(107)$ \\
\hline 62 & $2 s^{2} 2 p^{5} 4 s^{3} p^{0}$ & 0 & 956.00403 & 956.2259 & & 955.9575 & $1.44 \mathrm{E}-14$ & 100 \\
\hline 63 & $2 s^{2} 2 p^{5} 4 s^{3} p^{0}$ & 1 & 956.072409 & 956.2947 & & 956.0265 & $1.12 \mathrm{E}-14$ & $66.42+33.41(39)$ \\
\hline 64 & $2 s^{2} 2 p^{5} 4 p^{3} D^{e}$ & 1 & 960.469893 & 960.6384 & & 960.4153 & 1.19E-14 & $63.96+22.37(43)$ \\
\hline 65 & $2 s^{2} 2 p^{5} 4 p^{3} p^{e}$ & 0 & 961.613996 & 961.7564 & & 961.5413 & $1.23 \mathrm{E}-14$ & $65.29+33.99(45)$ \\
\hline 66 & $2 s^{2} 2 p^{5} 5 s^{3} p^{0}$ & 2 & 963.020297 & 963.1435 & & 962.9295 & $1.79 \mathrm{E}-14$ & 100 \\
\hline 67 & $2 s^{2} 2 p^{5} 5 s^{1} p^{0}$ & 1 & 963.119652 & 963.2415 & & 963.0282 & $1.13 \mathrm{E}-14$ & $66.59+33.41(155)$ \\
\hline 68 & $2 s^{2} 2 p^{5} 5 p^{3} p^{e}$ & 1 & 965.320423 & 965.4146 & & 965.2246 & $1.49 \mathrm{E}-14$ & $49.84+31.14(158)$ \\
\hline 69 & $2 s^{2} 2 p^{5} 5 p^{3} D^{e}$ & 2 & 965.34129 & 965.4353 & & 965.2449 & 1.49E-14 & $49.98+33.41(159)+16.48(72)$ \\
\hline 70 & $2 s^{2} 2 p^{5} 5 p^{1} p^{e}$ & 1 & 971.061253 & 971.1711 & & 970.9703 & $2.16 \mathrm{E}-14$ & $56.25+35.28(158)$ \\
\hline 71 & $2 s^{2} 2 p^{5} 5 p^{3} D^{e}$ & 3 & 971.080881 & 971.1864 & & 970.9884 & $2.22 \mathrm{E}-14$ & 100 \\
\hline 72 & $2 s^{2} 2 p^{5} 5 p^{3} p^{e}$ & 2 & 971.255626 & 971.3576 & & 971.1606 & $2.23 \mathrm{E}-14$ & $66.75+33.18(159)$ \\
\hline 73 & $2 s^{2} 2 p^{5} 5 p^{1} s^{e}$ & 0 & 971.97341 & 972.0335 & & 971.8575 & $2.28 \mathrm{E}-14$ & $65.61+33.64(157)$ \\
\hline 74 & $2 s^{2} 2 p^{5} 4 p^{3} s^{e}$ & 1 & 972.113217 & 972.2961 & & 972.0601 & $1.90 \mathrm{E}-14$ & $49.84+29.05(74)$ \\
\hline 75 & $2 s^{2} 2 p^{5} 4 p{ }^{1} D^{e}$ & 2 & 972.126624 & 972.3156 & & 973.1947 & $1.86 \mathrm{E}-14$ & $49.84+33.41(75)+16.73(44)$ \\
\hline 76 & $2 s^{2} 2 p^{5} 5 d{ }^{3} p^{0}$ & 0 & 973.288641 & 973.3864 & & 972.0758 & $1.09 \mathrm{E}-14$ & 100 \\
\hline 77 & $2 s^{2} 2 p^{5} 5 d^{3} p^{0}$ & 1 & 973.413991 & 973.5094 & & 973.3189 & $8.91 \mathrm{E}-15$ & $64+33.87(161)$ \\
\hline 78 & $2 s^{2} 2 p^{5} 5 d^{3} F^{0}$ & 3 & 973.416114 & 973.5106 & & 973.3201 & $1.10 \mathrm{E}-14$ & $53.44+39.56(163)$ \\
\hline 79 & $2 s^{2} 2 p^{5} 5 d^{3} D^{0}$ & 2 & 973.51952 & 973.6106 & & 973.4218 & $1.10 \mathrm{E}-14$ & $54.46+18.15(160)+18.06(81)$ \\
\hline 80 & $2 s^{2} 2 p^{5} 5 d^{3} F^{0}$ & 4 & 974.794167 & 974.8949 & & 974.7015 & $1.09 \mathrm{E}-14$ & 100 \\
\hline 81 & $2 s^{2} 2 p^{5} 5 d{ }^{1} D^{0}$ & 2 & 974.878976 & 974.9769 & & 974.7847 & $1.08 \mathrm{E}-14$ & $48.44+40.83(162)$ \\
\hline 82 & $2 s^{2} 2 p^{5} 5 d^{3} D^{0}$ & 3 & 975.01672 & 975.1095 & & 974.9196 & $1.09 \mathrm{E}-14$ & $70.73+27.05(163)$ \\
\hline 83 & $2 s^{2} 2 p^{5} 5 d^{1} p^{0}$ & 1 & 975.254656 & 975.3433 & & 975.1580 & $3.10 \mathrm{E}-15$ & $62.41+18.75(77)$ \\
\hline 84 & $2 s^{2} 2 p^{5} 5 f^{3} D^{e}$ & 1 & 976.064266 & 976.1707 & & 975.9780 & $7.16 \mathrm{E}-15$ & 100 \\
\hline 85 & $2 s^{2} 2 p^{5} 5 f^{3} G^{e}$ & 4 & 976.08678 & 976.1929 & & 976.0023 & $7.29 E-15$ & $53+43.82(167)$ \\
\hline 86 & $2 s^{2} 2 p^{5} 5 f^{3} F^{e}$ & 2 & 976.156799 & 976.262 & & 976.0701 & $7.18 \mathrm{E}-15$ & $47.33+47.2(165)$ \\
\hline 87 & $2 s^{2} 2 p^{5} 5 f^{3} F^{e}$ & 3 & 976.199984 & 976.3043 & & 976.1131 & $7.24 \mathrm{E}-15$ & $50.69+22.66(164)+22.37(90)$ \\
\hline 88 & $2 s^{2} 2 p^{5} 5 f^{3} G^{e}$ & 5 & 976.717308 & 976.824 & & 976.6329 & $7.49 \mathrm{E}-15$ & 100 \\
\hline 89 & $2 s^{2} 2 p^{5} 5 f{ }^{1} D^{e}$ & 2 & 976.797146 & 976.9016 & & 976.7120 & $7.28 \mathrm{E}-15$ & $61.15+30.58(165)$ \\
\hline 90 & $2 s^{2} 2 p^{5} 5 f^{1} F^{e}$ & 3 & 976.805504 & 976.9068 & & 976.7190 & $7.43 E-15$ & $44.22+40.32(166)$ \\
\hline 91 & $2 s^{2} 2 p^{5} 5 f^{3} F^{e}$ & 4 & 976.849551 & 976.9106 & & 976.7631 & $7.48 \mathrm{E}-15$ & $71.91+22.85(167)$ \\
\hline 92 & $2 s^{2} 2 p^{5} 5 g^{3} F^{0}$ & 2 & 976.923821 & 976.9542 & & 976.8410 & $1.27 \mathrm{E}-14$ & 99.8 \\
\hline 93 & $2 s^{2} 2 p^{5} 5 g{ }^{3} H^{0}$ & 5 & 976.921396 & 977.0168 & & 976.8384 & $1.28 \mathrm{E}-14$ & $52.56+45.83(170)$ \\
\hline 94 & $2 s^{2} 2 p^{5} 5 g{ }^{3} G^{0}$ & 3 & 976.970113 & 977.0294 & & 976.8867 & $1.29 \mathrm{E}-14$ & $54.32+36.72(169)$ \\
\hline 95 & $2 s^{2} 2 p^{5} 5 g^{3} G^{0}$ & 4 & 976.988488 & 977.066 & & 976.9052 & $1.28 \mathrm{E}-14$ & $47.47+25.2(168)+25(98)$ \\
\hline 96 & $2 s^{2} 2 p^{5} 5 g^{1} F^{0}$ & 3 & 977.304296 & 977.0843 & & 977.2209 & $1.30 \mathrm{E}-14$ & $57.61+38.32(169)$ \\
\hline 97 & $2 s^{2} 2 p^{5} 5 g{ }^{3} H^{0}$ & 6 & 977.305464 & 977.3999 & & 977.2225 & 1.30E-14 & 100 \\
\hline 98 & $2 s^{2} 2 p^{5} 5 g^{1} G^{0}$ & 4 & 977.356095 & 977.4009 & & 977.2727 & $1.30 \mathrm{E}-14$ & $41.6+39.44(171)+17.47(95)$ \\
\hline 99 & $2 s^{2} 2 p^{5} 5 g^{3} G^{0}$ & 5 & 977.371042 & 977.452 & & 977.2879 & $1.30 \mathrm{E}-14$ & $71.57+20.88(170)$ \\
\hline 100 & $2 s^{2} 2 p^{5} 4 d^{3} F^{0}$ & 2 & 976.756339 & 977.4669 & & 976.6994 & $8.26 \mathrm{E}-15$ & $74.3+20.07(51)$ \\
\hline 101 & $2 s^{2} 2 p^{5} 4 d^{3} D^{0}$ & 1 & 977.519699 & 977.648 & & 977.4548 & $9.92 \mathrm{E}-16$ & $48.02+33.29(53)$ \\
\hline
\end{tabular}




\begin{tabular}{|c|c|c|c|c|c|c|c|}
\hline 102 & $2 s^{2} 2 p^{5} 4 d^{3} p^{0}$ & 2 & 979.629004 & 979.794 & 979.5743 & $7.87 \mathrm{E}-15$ & $47.61+35.76(49)$ \\
\hline 103 & $2 s^{2} 2 p^{5} 4 d{ }^{1} F^{0}$ & 3 & 979.70381 & 979.8675 & 979.6484 & $7.91 \mathrm{E}-15$ & $44.22+33.41(103)+22.28(52)$ \\
\hline 104 & $2 s^{2} 2 p^{5} 4 f^{3} G^{e}$ & 3 & 982.214728 & 982.3897 & 982.1811 & $3.84 \mathrm{E}-15$ & $73.44+19.1(60)$ \\
\hline 105 & $2 s^{2} 2 p^{5} 4 f^{3} D^{e}$ & 2 & 982.283782 & 982.4584 & & $3.78 \mathrm{E}-15$ & $44.62+33.29(59)+22.09(105)$ \\
\hline 106 & $2 s^{2} 2 p^{5} 4 f^{3} D^{e}$ & 3 & 983.494741 & 983.6619 & 983.4601 & $3.92 \mathrm{E}-15$ & $54.32+29.81(57)$ \\
\hline 107 & $2 s^{2} 2 p^{5} 4 f^{1} G^{e}$ & 4 & 983.487779 & 983.6692 & 983.4543 & 3.95E-15 & $41.6+33.29(107)+25.1(61)$ \\
\hline 108 & $2 s 2 p^{6} 4 s{ }^{3} s^{e}$ & 1 & 992.0433 & 991.8102 & 991.8033 & $1.09 \mathrm{E}-14$ & 99.8 \\
\hline 109 & $2 s 2 p^{6} 4 s^{1} s^{e}$ & 0 & 992.862836 & 992.5901 & 992.5874 & $1.09 \mathrm{E}-14$ & 100 \\
\hline 110 & $2 s 2 p^{6} 4 p{ }^{3} p^{0}$ & 0 & 996.659716 & 996.3708 & 996.4039 & $9.45 \mathrm{E}-15$ & 100 \\
\hline 111 & $2 s 2 p^{6} 4 p^{3} p^{0}$ & 1 & 996.68872 & 996.3998 & 996.4343 & 4.00E-15 & $67.57+32.38(113)$ \\
\hline 112 & $2 s 2 p^{6} 4 p{ }^{3} p^{0}$ & 2 & 1008.15002 & 1007.887 & 1007.9087 & $1.32 \mathrm{E}-14$ & 100 \\
\hline 113 & $2 s 2 p^{6} 4 p{ }^{1} p^{0}$ & 1 & 1008.30862 & 1008.042 & 1008.0598 & $3.19 \mathrm{E}-15$ & $67.57+32.38(111)$ \\
\hline 114 & $2 s 2 p^{6} 4 d^{3} D^{e}$ & 1 & 1012.80724 & 1012.503 & 1012.5546 & $6.89 \mathrm{E}-15$ & 100 \\
\hline 115 & $2 s 2 p^{6} 4 d^{3} D^{e}$ & 2 & 1012.94302 & 1012.639 & 1012.6918 & $6.85 \mathrm{E}-15$ & $68.23+31.7(117)$ \\
\hline 116 & $2 s 2 p^{6} 4 d^{3} D^{e}$ & 3 & 1015.5612 & 1015.273 & 1015.3183 & $6.67 \mathrm{E}-15$ & 100 \\
\hline 117 & $2 s 2 p^{6} 4 d^{1} D^{e}$ & 2 & 1015.96123 & 1015.66 & 1015.7085 & $6.60 \mathrm{E}-15$ & $68.23+31.7(115)$ \\
\hline 118 & $2 s 2 p^{6} 4 f{ }^{3} F^{0}$ & 2 & 1018.11316 & 1017.826 & 1017.8997 & $3.51 \mathrm{E}-15$ & 100 \\
\hline 119 & $2 s 2 p^{6} 4 f^{3} F^{0}$ & 3 & 1018.09395 & 1017.836 & 1017.8808 & $3.52 \mathrm{E}-15$ & $60.06+39.94(121)$ \\
\hline 120 & $2 s 2 p^{6} 4 f^{3} F^{0}$ & 4 & 1019.32221 & 1019.054 & 1019.1094 & 3.60E-15 & 100 \\
\hline 121 & $2 s 2 p^{6} 4 f^{1} F^{0}$ & 3 & 1019.40822 & 1019.13 & 1019.1955 & $3.61 \mathrm{E}-15$ & $60.06+39.94(119)$ \\
\hline 122 & $2 s^{2} 2 p^{5} 6 s^{3} p^{0}$ & 2 & 1020.96254 & 1021.039 & & $2.54 \mathrm{E}-14$ & 100 \\
\hline 123 & $2 s^{2} 2 p^{5} 6 s^{1} p^{0}$ & 1 & 1021.01233 & 1021.085 & & $1.82 \mathrm{E}-14$ & $66.59+33.41(191)$ \\
\hline 124 & $2 s^{2} 2 p^{5} 6 p^{3} p^{e}$ & 1 & 1022.27224 & 1022.333 & & $2.13 \mathrm{E}-14$ & $49.84+30.8(194)$ \\
\hline 125 & $2 s^{2} 2 p^{5} 6 p^{3} D^{e}$ & 2 & 1022.28233 & 1022.358 & & $2.12 \mathrm{E}-14$ & $49.98+33.41(195)+16.56(128)$ \\
\hline 126 & $2 s^{2} 2 p^{5} 6 p^{1} p^{e}$ & 1 & 1025.57363 & 1025.683 & & $2.99 \mathrm{E}-14$ & $56.25+35.88(194)$ \\
\hline 127 & $2 s^{2} 2 p^{5} 6 p^{3} D^{e}$ & 3 & 1025.56699 & 1025.683 & & $3.06 \mathrm{E}-14$ & 100 \\
\hline 128 & $2 s^{2} 2 p^{5} 6 p^{3} p^{e}$ & 2 & 1025.65942 & 1025.761 & & $3.04 \mathrm{E}-14$ & $66.74+33.18(195)$ \\
\hline 129 & $2 s^{2} 2 p^{5} 6 p^{1} s^{e}$ & 0 & 1026.01886 & 1026.086 & & $2.95 \mathrm{E}-14$ & $66.42+33.52(193)$ \\
\hline 130 & $2 s^{2} 2 p^{5} 6 d^{3} p^{0}$ & 0 & 1026.81572 & 1026.917 & & $1.63 \mathrm{E}-14$ & 100 \\
\hline 131 & $2 s^{2} 2 p^{5} 6 d^{3} p^{0}$ & 1 & 1026.88419 & 1027.007 & & $1.41 \mathrm{E}-14$ & $62.57+34.69(197)$ \\
\hline 132 & $2 s^{2} 2 p^{5} 6 d^{3} F^{0}$ & 3 & 1026.88383 & 1027.025 & & $1.63 \mathrm{E}-14$ & $53.44+39.56(199)$ \\
\hline 133 & $2 s^{2} 2 p^{5} 6 d^{3} D^{0}$ & 2 & 1026.94072 & 1027.061 & & $1.63 \mathrm{E}-14$ & $54.32+18.4(135)+18.23(196)$ \\
\hline 134 & $2 s^{2} 2 p^{5} 6 d^{3} F^{0}$ & 4 & 1027.68395 & 1027.845 & & $1.63 \mathrm{E}-14$ & 100 \\
\hline 135 & $2 s^{2} 2 p^{5} 6 d^{1} D^{0}$ & 2 & 1027.7356 & 1027.88 & & $1.63 \mathrm{E}-14$ & $48.16+41.09(198)$ \\
\hline 136 & $2 s^{2} 2 p^{5} 6 d^{3} D^{0}$ & 3 & 1027.80544 & 1027.948 & & 1.63E-14 & $70.9+27.04(199)$ \\
\hline 137 & $2 s^{2} 2 p^{5} 6 d^{1} p^{0}$ & 1 & 1028.0006 & 1028.136 & & $3.07 \mathrm{E}-15$ & $63.84+20.79(131)$ \\
\hline 138 & $2 s^{2} 2 p^{5} 7 s^{3} p^{0}$ & 2 & 1055.24314 & 1055.369 & & $3.48 \mathrm{E}-14$ & 100 \\
\hline 139 & $2 s^{2} 2 p^{5} 7 s^{1} p^{0}$ & 1 & 1055.27514 & 1055.398 & & $2.66 \mathrm{E}-14$ & $66.59+33.29(201)$ \\
\hline 140 & $2 s^{2} 2 p^{5} 7 p^{3} p^{e}$ & 1 & 1056.06027 & 1056.176 & & $2.94 \mathrm{E}-14$ & $49.98+30.8(204)$ \\
\hline 141 & $2 s^{2} 2 p^{5} 7 p^{3} D^{e}$ & 2 & 1056.0654 & 1056.191 & & $2.93 \mathrm{E}-14$ & $49.98+33.41(205)+16.56(144)$ \\
\hline 142 & $2 s^{2} 2 p^{5} 7 p^{3} D^{e}$ & 3 & 1058.11679 & 1058.267 & & $4.06 \mathrm{E}-14$ & 100 \\
\hline 143 & $2 s^{2} 2 p^{5} 7 p^{1} p^{e}$ & 1 & 1058.12109 & 1058.267 & & $3.90 \mathrm{E}-14$ & $56.4+35.88(204)$ \\
\hline 144 & $2 s^{2} 2 p^{5} 7 p^{3} p^{e}$ & 2 & 1058.17486 & 1058.316 & & $4.01 \mathrm{E}-14$ & $66.75+33.18(205)$ \\
\hline 145 & $2 s^{2} 2 p^{5} 7 p^{1} s^{e}$ & 0 & 1058.38657 & 1058.504 & & 3.77E-14 & $66.42+33.41(203)$ \\
\hline 146 & $2 s^{2} 2 p^{5} 7 d^{3} p^{0}$ & 0 & 1058.89328 & 1059.037 & & $2.36 \mathrm{E}-14$ & 100 \\
\hline 147 & $2 s^{2} 2 p^{5} 7 d^{3} p^{0}$ & 1 & 1058.93537 & 1059.091 & & $2.06 \mathrm{E}-14$ & $62.73+34.69(207)$ \\
\hline 148 & $2 s^{2} 2 p^{5} 7 d^{3} F^{0}$ & 3 & 1058.93476 & 1059.101 & & $2.35 \mathrm{E}-14$ & $53.44+39.69(209)$ \\
\hline 149 & $2 s^{2} 2 p^{5} 7 d^{3} D^{0}$ & 2 & 1058.96984 & 1059.123 & & $2.34 \mathrm{E}-14$ & $54.32+18.4(151)+18.23(206)$ \\
\hline 150 & $2 s^{2} 2 p^{5} 7 d^{3} F^{0}$ & 4 & 1059.43945 & 1059.619 & & $2.40 \mathrm{E}-14$ & 100 \\
\hline 151 & $2 s^{2} 2 p^{5} 7 d^{1} D^{0}$ & 2 & 1059.47086 & 1059.64 & & 2.37E-14 & $48.3+41.09(208)$ \\
\hline 152 & $2 s^{2} 2 p^{5} 7 d^{3} D^{0}$ & 3 & 1059.51416 & 1059.681 & & $2.36 \mathrm{E}-14$ & $70.9+27.04(209)$ \\
\hline 153 & $2 s^{2} 2 p^{5} 7 d^{1} p^{0}$ & 1 & 1059.63188 & 1059.792 & & $5.17 \mathrm{E}-15$ & $63.84+20.7(147)$ \\
\hline 154 & $2 s^{2} 2 p^{5} 5 s^{3} p^{0}$ & 0 & 1065.02071 & 1060.053 & & $1.79 \mathrm{E}-14$ & 100 \\
\hline 155 & $2 s^{2} 2 p^{5} 5 s^{3} p^{0}$ & 1 & 1065.05207 & 1060.076 & & $1.54 \mathrm{E}-14$ & $66.59+33.41(67)$ \\
\hline 156 & $2 s^{2} 2 p^{5} 5 p^{3} D^{e}$ & 1 & 1067.26502 & 1067.464 & & $1.49 \mathrm{E}-14$ & $73.96+22.28(70)$ \\
\hline 157 & $2 s^{2} 2 p^{5} 5 p^{3} p^{e}$ & 0 & 1067.83295 & 1068.009 & & $1.51 \mathrm{E}-14$ & $66.1+33.76(73)$ \\
\hline 158 & $2 s^{2} 2 p^{5} 5 p^{3} s^{e}$ & 1 & 1073.11563 & 1073.324 & & $2.23 \mathrm{E}-14$ & $50.13+29.48(158)$ \\
\hline 159 & $2 s^{2} 2 p^{5} 5 p^{1} D^{e}$ & 2 & 1073.12818 & 1073.337 & & $2.20 \mathrm{E}-14$ & $49.98+33.41(159)+16.65(72)$ \\
\hline 160 & $2 s^{2} 2 p^{5} 5 d^{3} F^{0}$ & 2 & 1075.39643 & 1075.597 & & 1.10E-14 & $74.65+20.07(81)$ \\
\hline 161 & $2 s^{2} 2 p^{5} 5 d^{3} D^{0}$ & 1 & 1075.71349 & 1075.899 & & $2.45 \mathrm{E}-15$ & $49.84+33.64(83)+16.48(77)$ \\
\hline 162 & $2 s^{2} 2 p^{5} 5 d^{3} p^{0}$ & 2 & 1076.87223 & 1077.075 & & $1.08 \mathrm{E}-14$ & $47.89+35.64(79)$ \\
\hline 163 & $2 s^{2} 2 p^{5} 5 d^{1} F^{0}$ & 3 & 1076.90822 & 1077.11 & & $1.09 \mathrm{E}-14$ & $44.36+33.41(163)+22.28(82)$ \\
\hline 164 & $2 s^{2} 2 p^{5} 5 f^{3} G^{e}$ & 3 & 1078.14744 & 1078.357 & & 7.27E-15 & $73.44+19.1(90)$ \\
\hline 165 & $2 s^{2} 2 p^{5} 5 f^{3} D^{e}$ & 2 & 1078.1903 & 1078.399 & & 7.12E-15 & $44.49+33.29(89)+22.18(165)$ \\
\hline 166 & $2 s^{2} 2 p^{5} 5 f^{3} D^{e}$ & 3 & 1078.80466 & 1079.013 & & 7.43E-15 & $54.32+29.81(87)$ \\
\hline 167 & $2 s^{2} 2 p^{5} 5 f^{1} G^{e}$ & 4 & 1078.80431 & 1079.014 & & $7.50 \mathrm{E}-15$ & $41.6+33.29(167)+25(91)$ \\
\hline 168 & $2 s^{2} 2 p^{5} 5 g^{3} H^{0}$ & 4 & 1078.98218 & 1079.181 & & $1.28 \mathrm{E}-14$ & $72.42+18.49(98)$ \\
\hline 169 & $2 s^{2} 2 p^{5} 5 g^{3} F^{0}$ & 3 & 1078.99025 & 1079.19 & & $1.28 \mathrm{E}-14$ & $41.73+33.29(96)+25(169)$ \\
\hline 170 & $2 s^{2} 2 p^{5} 5 g^{1} H^{0}$ & 5 & 1079.36669 & 1079.566 & & $1.30 \mathrm{E}-14$ & $39.94+33.29(170)+26.73(99)$ \\
\hline 171 & $2 s^{2} 2 p^{5} 5 g^{3} F^{0}$ & 4 & 1079.37255 & 1079.572 & & $1.29 \mathrm{E}-14$ & $57.61+26.63(95)$ \\
\hline 172 & $2 s 2 p^{6} 5 s^{3} s^{e}$ & 1 & 1100.9399 & 1100.731 & & $1.24 \mathrm{E}-14$ & 100 \\
\hline
\end{tabular}




\begin{tabular}{|c|c|c|c|c|c|c|}
\hline 173 & $2 s 2 p^{6} 5 s^{1} s^{e}$ & 0 & 1101.33244 & 1101.094 & $1.24 \mathrm{E}-14$ & 100 \\
\hline 174 & $2 s 2 p^{6} 5 p^{3} p^{0}$ & 0 & 1103.26766 & 1103.025 & $1.09 \mathrm{E}-14$ & 100 \\
\hline 175 & $2 s 2 p^{6} 5 p^{3} p^{0}$ & 1 & 1103.28033 & 1103.038 & $5.84 \mathrm{E}-15$ & $67.57+32.49(177)$ \\
\hline 176 & $2 s 2 p^{6} 5 p^{3} p^{0}$ & 2 & 1109.05738 & 1108.829 & $1.44 \mathrm{E}-14$ & 100 \\
\hline 177 & $2 s 2 p^{6} 5 p^{1} p^{0}$ & 1 & 1109.13028 & 1108.899 & $4.91 \mathrm{E}-15$ & $67.57+32.49(175)$ \\
\hline 178 & $2 s 2 p^{6} 5 d^{3} D^{e}$ & 1 & 1111.33642 & 1111.1 & $8.62 \mathrm{E}-15$ & 100 \\
\hline 179 & $2 s 2 p^{6} 5 d^{3} D^{e}$ & 2 & 1111.40589 & 1111.165 & $8.55 \mathrm{E}-15$ & $67.73+32.15(181)$ \\
\hline 180 & $2 s 2 p^{6} 5 d^{3} D^{e}$ & 3 & 1112.7597 & 1112.53 & $8.55 \mathrm{E}-15$ & 100 \\
\hline 181 & $2 s 2 p^{6} 5 d^{1} D^{e}$ & 2 & 1112.94781 & 1112.707 & $8.46 \mathrm{E}-15$ & $67.73+32.15(179)$ \\
\hline 182 & $2 s 2 p^{6} 5 f^{3} F^{0}$ & 2 & 1114.00972 & 1113.794 & $6.17 E-15$ & 100 \\
\hline 183 & $2 s 2 p^{6} 5 f^{3} F^{0}$ & 3 & 1114.00895 & 1113.797 & $6.16 \mathrm{E}-15$ & $61+38.94(185)$ \\
\hline 184 & $2 s 2 p^{6} 5 f^{3} F^{0}$ & 4 & 1114.63499 & 1114.421 & $6.32 \mathrm{E}-15$ & 100 \\
\hline 185 & $2 s 2 p^{6} 5 f^{1} F^{0}$ & 3 & 1114.68342 & 1114.47 & $6.36 \mathrm{E}-15$ & $61+38.94(183)$ \\
\hline 186 & $2 s 2 p^{6} 5 g{ }^{3} G^{e}$ & 3 & 1114.82296 & 1114.591 & $9.99 \mathrm{E}-15$ & 100 \\
\hline 187 & $2 s 2 p^{6} 5 g{ }^{3} G^{e}$ & 4 & 1114.81033 & 1114.607 & $9.93 \mathrm{E}-15$ & $55.8+44.22(189)$ \\
\hline 188 & $2 s 2 p^{6} 5 g^{3} G^{e}$ & 5 & 1115.1943 & 1114.975 & $1.00 \mathrm{E}-14$ & 100 \\
\hline 189 & $2 s 2 p^{6} 5 g^{1} G^{e}$ & 4 & 1115.20628 & 1114.99 & $1.01 \mathrm{E}-14$ & $55.8+44.22(187)$ \\
\hline 190 & $2 s^{2} 2 p^{5} 6 s^{3} p^{0}$ & 0 & 1122.93735 & 1123.204 & $2.53 \mathrm{E}-14$ & 100 \\
\hline 191 & $2 s^{2} 2 p^{5} 6 s^{3} p^{0}$ & 1 & 1122.95313 & 1123.23 & $2.22 \mathrm{E}-14$ & $66.59+33.41(123)$ \\
\hline 192 & $2 s^{2} 2 p^{5} 6 p^{3} D^{e}$ & 1 & 1124.21573 & 1124.493 & $2.11 \mathrm{E}-14$ & $73.96+22.28(126)$ \\
\hline 193 & $2 s^{2} 2 p^{5} 6 p^{3} p^{e}$ & 0 & 1124.51121 & 1124.761 & $2.12 \mathrm{E}-14$ & $66.42+33.52(129)$ \\
\hline 194 & $2 s^{2} 2 p^{5} 6 p^{3} s^{e}$ & 1 & 1127.56301 & 1127.858 & $3.05 \mathrm{E}-14$ & $49.98+29.59(194)$ \\
\hline 195 & $2 s^{2} 2 p^{5} 6 p^{1} D^{e}$ & 2 & 1127.56736 & 1127.881 & 3.02E-14 & $49.98+33.29(195)+16.65(128)$ \\
\hline 196 & $2 s^{2} 2 p^{5} 6 d^{3} F^{0}$ & 2 & 1128.84881 & 1129.183 & $1.64 \mathrm{E}-14$ & $74.65+19.98(135)$ \\
\hline 197 & $2 s^{2} 2 p^{5} 6 d^{3} D^{0}$ & 1 & 1129.02081 & 1129.353 & $4.35 \mathrm{E}-15$ & $49.84+33.52(137)+16.56(131)$ \\
\hline 198 & $2 s^{2} 2 p^{5} 6 d^{3} p^{0}$ & 2 & 1129.70477 & 1130.047 & $1.63 \mathrm{E}-14$ & $47.89+35.64(133)$ \\
\hline 199 & $2 s^{2} 2 p^{5} 6 d^{1} F^{0}$ & 3 & 1129.72338 & 1130.074 & $1.63 \mathrm{E}-14$ & $44.36+33.29(199)+22.28(136)$ \\
\hline 200 & $2 s^{2} 2 p^{5} 7 s^{3} p^{0}$ & 0 & 1157.22746 & 1157.541 & $3.49 \mathrm{E}-14$ & 100 \\
\hline 201 & $2 s^{2} 2 p^{5} 7 s^{3} p^{0}$ & 1 & 1157.23689 & 1157.561 & $3.14 \mathrm{E}-14$ & $66.59+33.41(139)$ \\
\hline 202 & $2 s^{2} 2 p^{5} 7 p^{3} D^{e}$ & 1 & 1158.02382 & 1158.343 & $2.93 \mathrm{E}-14$ & $74.13+22.28(143)$ \\
\hline 203 & $2 s^{2} 2 p^{5} 7 p^{3} p^{e}$ & 0 & 1158.20465 & 1158.509 & $2.89 \mathrm{E}-14$ & $66.59+33.52(145)$ \\
\hline 204 & $2 s^{2} 2 p^{5} 7 p^{3} s^{e}$ & 1 & 1160.11335 & 1158.609 & $4.05 \mathrm{E}-14$ & $49.98+29.59(204)$ \\
\hline 205 & $2 s^{2} 2 p^{5} 7 p^{1} D^{e}$ & 2 & 1160.1162 & 1158.803 & $4.03 E-14$ & $49.98+33.29(205)+16.65(144)$ \\
\hline 206 & $2 s^{2} 2 p^{5} 7 d^{3} F^{0}$ & 2 & 1160.91151 & 1159.928 & $2.37 \mathrm{E}-14$ & $74.65+19.98(151)$ \\
\hline 207 & $2 s^{2} 2 p^{5} 7 d^{3} D^{0}$ & 1 & 1161.01728 & 1159.941 & $7.01 \mathrm{E}-15$ & $49.98+33.41(153)+16.65(147)$ \\
\hline 208 & $2 s^{2} 2 p^{5} 7 d^{3} p^{0}$ & 2 & 1161.45028 & 1160.461 & $2.39 \mathrm{E}-14$ & $47.89+35.64(149)$ \\
\hline 209 & $2 s^{2} 2 p^{5} 7 d^{1} F^{0}$ & 3 & 1161.46181 & 1160.464 & $2.39 \mathrm{E}-14$ & $44.36+33.29(209)+22.28(152)$ \\
\hline
\end{tabular}

Table 4: MCDF energies (in Ryd.), lifetimes (in s) and mixing coefficients in Ne-like-Re

\begin{tabular}{|c|c|c|c|c|c|c|}
\hline S.No. & Label & $\mathrm{J}$ & $\begin{array}{l}\text { Level } \\
\text { (Ryd) }\end{array}$ & FAC & Lifetimes & Mixing coefficients \\
\hline 1 & $2 s^{2} 2 p^{61} s^{e}$ & 0 & $\ldots \ldots \ldots$ & 0 & ......... & 100 \\
\hline 2 & $2 s^{2} 2 p^{5} 3 s^{13} p^{0}$ & 2 & 626.8857 & 627.0489 & $2.03911 \mathrm{E}-10$ & 100 \\
\hline 3 & $2 s^{2} 2 p^{5} 3 s^{11} p^{0}$ & 1 & 627.5184 & 627.6812 & $6.06722 \mathrm{E}-15$ & $66.3+33.6(4)$ \\
\hline 4 & $2 s^{2} 2 p^{5} 3 p^{13} p^{e}$ & 1 & 638.2366 & 638.2437 & $9.23808 \mathrm{E}-13$ & $49.4+31.5(17)$ \\
\hline 5 & $2 s^{2} 2 p^{5} 3 p^{13} D^{e}$ & 2 & 638.3612 & 638.3671 & $9.55771 \mathrm{E}-13$ & $50.3+33.3(15)+16.5(8)$ \\
\hline 6 & $2 s^{2} 2 p^{5} 3 p^{11} p^{e}$ & 1 & 667.9257 & 667.9529 & $5.53778 \mathrm{E}-13$ & $56+36(17)$ \\
\hline 7 & $2 s^{2} 2 p^{5} 3 p^{13} D^{e}$ & 3 & 667.8903 & 667.9841 & $5.29884 \mathrm{E}-13$ & 100 \\
\hline 8 & $2 s^{2} 2 p^{5} 3 p^{13} p^{e}$ & 2 & 668.8759 & 668.9274 & 3.33524E-13 & $66.7+33.3(15)$ \\
\hline 9 & $2 s^{2} 2 p^{5} 3 p^{11} s^{e}$ & 0 & 673.2555 & 673.2283 & 3.64834E-13 & $62.4+37.5(16)$ \\
\hline 10 & $2 s^{2} 2 p^{5} 3 d^{13} p^{0}$ & 0 & 679.8178 & 679.7983 & $7.27856 \mathrm{E}-13$ & 100 \\
\hline 11 & $2 s^{2} 2 p^{5} 3 d^{13} p^{0}$ & 1 & 680.5647 & 680.544 & $1.07775 \mathrm{E}-14$ & $65.6+32.6(9)$ \\
\hline 12 & $2 s^{2} 2 p^{5} 3 d^{13} F^{0}$ & 3 & 680.615 & 680.5948 & $6.94131 \mathrm{E}-13$ & $53.9+39.2(3)$ \\
\hline 13 & $2 s^{2} 2 p^{5} 3 d^{13} D^{0}$ & 2 & 681.2308 & 681.2044 & $6.62026 \mathrm{E}-13$ & $54.3+18.3(10)+17.6(117)$ \\
\hline 14 & $2 s^{2} 2 p^{5} 3 d^{13} F^{0}$ & 4 & 687.1255 & 687.133 & $5.99405 \mathrm{E}-12$ & 100 \\
\hline 15 & $2 s^{2} 2 p^{5} 3 d^{11} D^{0}$ & 2 & 687.6663 & 687.6557 & 3.22224E-12 & $48.4+41.2(116)$ \\
\hline 16 & $2 s^{2} 2 p^{5} 3 d^{13} D^{0}$ & 3 & 688.4206 & 688.4042 & $5.4829 \mathrm{E}-12$ & $70.6+27.2(3)$ \\
\hline 17 & $2 s^{2} 2 p^{5} 3 d^{11} p^{0}$ & 1 & 690.6284 & 690.5645 & 3.38737E-16 & $62.9+18.6(12)+18.5(9)$ \\
\hline 18 & $2 s^{2} 2 p^{5} 3 s^{13} p^{0}$ & 0 & 735.2385 & 735.5043 & $2.64685 \mathrm{E}-11$ & 99.8 \\
\hline 19 & $2 s^{2} 2 p^{5} 3 s^{13} p^{0}$ & 1 & 735.486 & 735.7518 & $2.37235 \mathrm{E}-14$ & $66.3+33.6(11)$ \\
\hline 20 & $2 s^{2} 2 p^{5} 3 p^{13} D^{e}$ & 1 & 746.2757 & 746.3916 & $1.37571 \mathrm{E}-11$ & $73.6+22.7(21)$ \\
\hline 21 & $2 s^{2} 2 p^{5} 3 p^{13} p^{e}$ & 0 & 749.9872 & 750.0719 & 2.77195E-12 & $62.4+37.3(23)$ \\
\hline 22 & $2 s^{1} 2 p^{6} 3 s^{13} s^{e}$ & 1 & 771.8 & 771.5597 & $4.45168 \mathrm{E}-14$ & 90.3 \\
\hline 23 & $2 s^{1} 2 p^{6} 3 s^{11} s^{e}$ & 0 & 775.0469 & 774.5994 & 4.09517E-14 & 99.8 \\
\hline
\end{tabular}




\begin{tabular}{|c|c|c|c|c|c|c|}
\hline 24 & $2 s^{1} 2 p^{5} 3 p^{11} D^{e}$ & 2 & 776.5715 & 776.7361 & $3.17953 \mathrm{E}-13$ & $49.7+33.5(15)+16.8(8)$ \\
\hline 25 & $2 s^{2} 2 p^{5} 3 p^{13} s^{e}$ & 1 & 777.094 & 777.1704 & $1.56339 \mathrm{E}-13$ & $45.3+26.8(17)$ \\
\hline 26 & $2 s^{1} 2 p^{6} 3 p^{13} p^{0}$ & 0 & 783.8923 & 783.322 & $3.8701 \mathrm{E}-14$ & 99.8 \\
\hline 27 & $2 s^{1} 2 p^{6} 3 p^{13} p^{0}$ & 1 & 783.7456 & 783.4371 & $1.27288 \mathrm{E}-15$ & $66.3+31.9(167)$ \\
\hline 28 & $2 s^{2} 2 p^{5} 3 d^{13} F^{0}$ & 2 & 788.9369 & 789.0352 & $6.62079 \mathrm{E}-13$ & $74.3+20.3(117)$ \\
\hline 29 & $2 s^{2} 2 p^{5} 3 d^{13} D^{0}$ & 1 & 790.9173 & 790.944 & $6.66626 \mathrm{E}-16$ & $48+34.7(121)$ \\
\hline 30 & $2 s^{2} 2 p^{5} 3 d^{13} p^{0}$ & 2 & 795.9605 & 796.0518 & $3.07266 \mathrm{E}-12$ & $47.1+36.1(14)$ \\
\hline 31 & $2 s^{2} 2 p^{5} 3 d^{11} F^{0}$ & 3 & 796.2127 & 796.3066 & $4.81625 \mathrm{E}-12$ & $44+33.5(19)+22.5(5)$ \\
\hline 32 & $2 s^{1} 2 p^{6} 3 p^{13} p^{0}$ & 2 & 813.6977 & 813.3061 & $3.59341 \mathrm{E}-14$ & 99.6 \\
\hline 33 & $2 s^{1} 2 p^{6} 3 p^{11} p^{0}$ & 1 & 814.1484 & 813.7534 & $2.00932 \mathrm{E}-15$ & $67.7+32.3(160)$ \\
\hline 34 & $2 s^{1} 2 p^{6} 3 d^{13} D^{e}$ & 1 & 826.0893 & 825.5601 & $4.01052 E-14$ & 100 \\
\hline 35 & $2 s^{1} 2 p^{6} 3 d^{13} D^{e}$ & 2 & 826.4821 & 825.9526 & $3.11414 \mathrm{E}-14$ & $70.1+29.9(136)$ \\
\hline 36 & $2 s^{1} 2 p^{6} 3 d^{13} D^{e}$ & 3 & 832.8699 & 832.3665 & $3.97477 \mathrm{E}-14$ & 100 \\
\hline 37 & $2 s^{1} 2 p^{6} 3 d^{11} D^{e}$ & 2 & 834.0198 & 833.5013 & $2.69074 \mathrm{E}-14$ & $70.1+29.9(137)$ \\
\hline 38 & $2 s^{2} 2 p^{5} 4 s^{13} p^{0}$ & 2 & 879.3475 & 879.47 & $1.35729 \mathrm{E}-14$ & 100 \\
\hline 39 & $2 s^{2} 2 p^{5} 4 s^{11} p^{0}$ & 1 & 879.5595 & 879.6826 & $7.55659 \mathrm{E}-15$ & $66.6+33.4(24)$ \\
\hline 40 & $2 s^{2} 2 p^{5} 4 p^{13} p^{e}$ & 1 & 884.0304 & 884.0945 & $1.124 \mathrm{E}-14$ & $49.7+31(28)$ \\
\hline 41 & $2 s^{2} 2 p^{5} 4 p^{13} D^{e}$ & 2 & 884.066 & 884.1305 & $1.11539 \mathrm{E}-14$ & $50.1+33.4(123)+16.5(125)$ \\
\hline 42 & $2 s^{2} 2 p^{5} 4 p^{13} D^{e}$ & 3 & 896.2112 & 896.2973 & $1.79119 \mathrm{E}-14$ & 100 \\
\hline 43 & $2 s^{2} 2 p^{5} 4 p^{11} p^{e}$ & 1 & 896.2364 & 896.3213 & $1.78223 \mathrm{E}-14$ & $56.3+35.9(28)$ \\
\hline 44 & $2 s^{2} 2 p^{5} 4 p^{13} p^{e}$ & 2 & 896.5753 & 896.6593 & $1.79821 \mathrm{E}-14$ & $66.7+33.2(123)$ \\
\hline 45 & $2 s^{2} 2 p^{5} 4 p^{11} s^{e}$ & 0 & 898.0605 & 898.0812 & $1.85584 \mathrm{E}-14$ & $65.6+34.3(37)$ \\
\hline 46 & $2 s^{2} 2 p^{5} 4 d^{13} p^{0}$ & 0 & 900.7814 & 900.8371 & $7.69049 \mathrm{E}-15$ & 100 \\
\hline 47 & $2 s^{2} 2 p^{5} 4 d^{13} p^{0}$ & 1 & 901.0504 & 901.1041 & $6.03592 \mathrm{E}-15$ & $63.7+34(42)$ \\
\hline 48 & $2 s^{2} 2 p^{5} 4 d^{13} F^{0}$ & 3 & 901.0548 & 901.1071 & $7.71813 \mathrm{E}-15$ & $53.6+39.6(41)$ \\
\hline 49 & $2 s^{2} 2 p^{5} 4 d^{13} D^{0}$ & 2 & 901.2791 & 901.3274 & $7.75333 \mathrm{E}-15$ & $54.3+18.2(53)+18.1(47)$ \\
\hline 50 & $2 s^{2} 2 p^{5} 4 d^{13} F^{0}$ & 4 & 903.8924 & 903.9546 & $7.41994 \mathrm{E}-15$ & 100 \\
\hline 51 & $2 s^{2} 2 p^{5} 4 d^{11} D^{0}$ & 2 & 904.0928 & 904.1506 & $7.41808 \mathrm{E}-15$ & $48.3+41.1(43)$ \\
\hline 52 & $2 s^{2} 2 p^{5} 4 d^{13} D^{0}$ & 3 & 904.364 & 904.4175 & $7.46398 \mathrm{E}-15$ & $70.7+27(41)$ \\
\hline 53 & $2 s^{2} 2 p^{5} 4 d^{11} p^{0}$ & 1 & 905.137 & 905.1656 & $8.34712 \mathrm{E}-16$ & $63.7+19.9(49)+16.3(42)$ \\
\hline 54 & $2 s^{2} 2 p^{5} 4 f^{13} D^{e}$ & 1 & 906.5339 & 906.603 & $3.52285 \mathrm{E}-15$ & 100 \\
\hline 55 & $2 s^{2} 2 p^{5} 4 f^{13} G^{e}$ & 4 & 906.5491 & 906.6185 & $3.61295 \mathrm{E}-15$ & $53+44.1(59)$ \\
\hline 56 & $2 s^{2} 2 p^{5} 4 f^{13} F^{e}$ & 2 & 906.7121 & 906.7813 & $3.56113 \mathrm{E}-15$ & $47.9+46(29)$ \\
\hline 57 & $2 s^{2} 2 p^{5} 4 f^{13} F^{e}$ & 3 & 906.7905 & 906.8597 & $3.60818 \mathrm{E}-15$ & $50.6+22.8(54)+22.6(56)$ \\
\hline 58 & $2 s^{2} 2 p^{5} 4 f^{13} G^{e}$ & 5 & 907.8632 & 907.9327 & 3.70932E-15 & 100 \\
\hline 59 & $2 s^{2} 2 p^{5} 4 f^{11} D^{e}$ & 2 & 907.9953 & 908.0621 & $3.60296 \mathrm{E}-15$ & $60.5+31.8(29)$ \\
\hline 60 & $2 s^{2} 2 p^{5} 4 f^{11} F^{e}$ & 3 & 908.0575 & 908.1262 & $3.67812 \mathrm{E}-15$ & $44+40.4(61)$ \\
\hline 61 & $2 s^{2} 2 p^{5} 4 f^{13} F^{e}$ & 4 & 908.1287 & 908.198 & $3.71949 \mathrm{E}-15$ & $71.9+22.6(59)$ \\
\hline 62 & $2 s^{2} 2 p^{5} 4 s^{13} p^{0}$ & 0 & 987.8259 & 988.0634 & $1.05282 \mathrm{E}-14$ & 100 \\
\hline 63 & $2 s^{2} 2 p^{5} 4 s^{13} p^{0}$ & 1 & 987.893 & 988.131 & $1.05289 \mathrm{E}-14$ & $66.4+33.4(22)$ \\
\hline 64 & $2 s^{2} 2 p^{5} 5 s^{13} p^{0}$ & 2 & 991.88 & 992.0083 & $1.6748 \mathrm{E}-14$ & 100 \\
\hline 65 & $2 s^{2} 2 p^{5} 5 s^{11} p^{0}$ & 1 & 991.9826 & 992.1094 & $1.06064 \mathrm{E}-14$ & $66.4+33.4(75)$ \\
\hline 66 & $2 s^{2} 2 p^{5} 4 p^{13} D^{e}$ & 1 & 992.3851 & 992.564 & $1.10869 \mathrm{E}-14$ & $74+22.4(30)$ \\
\hline 67 & $2 s^{2} 2 p^{5} 5 p^{13} p^{e}$ & 1 & 994.2316 & 993.6892 & $1.39622 \mathrm{E}-14$ & $49.8+31(81)$ \\
\hline 68 & $2 s^{2} 2 p^{5} 5 p^{13} D^{e}$ & 2 & 994.2504 & 994.328 & $1.39266 \mathrm{E}-14$ & $50+33.4(79)+16.5(72)$ \\
\hline 69 & $2 s^{2} 2 p^{5} 4 p^{13} p^{e}$ & 0 & 993.5349 & 994.3467 & $1.14749 \mathrm{E}-14$ & $65+33.8(33)$ \\
\hline 70 & $2 s^{2} 2 p^{5} 5 p^{11} p^{e}$ & 1 & 1000.372 & 1000.477 & $2.06199 \mathrm{E}-14$ & $56.4+35.6(81)$ \\
\hline 71 & $2 s^{2} 2 p^{5} 5 p^{13} D^{e}$ & 3 & 1000.368 & 1000.48 & $2.101 \mathrm{E}-14$ & 100 \\
\hline 72 & $2 s^{2} 2 p^{5} 5 p^{13} p^{e}$ & 2 & 1000.547 & 1000.652 & $2.10976 \mathrm{E}-14$ & $66.7+33.2(79)$ \\
\hline 73 & $2 s^{2} 2 p^{5} 5 p^{11} s^{e}$ & 0 & 1001.299 & 1001.36 & $2.1482 \mathrm{E}-14$ & $65.1+33.5(80)$ \\
\hline 74 & $2 s^{2} 2 p^{5} 5 d^{13} p^{0}$ & 0 & 1002.614 & 1002.714 & $1.02762 \mathrm{E}-14$ & 100 \\
\hline 75 & $2 s^{2} 2 p^{5} 5 d^{13} p^{0}$ & 1 & 1002.742 & 1002.839 & $8.48864 \mathrm{E}-15$ & $63.5+34.1(92)$ \\
\hline 76 & $2 s^{2} 2 p^{5} 5 d^{13} F^{0}$ & 3 & 1002.742 & 1002.84 & $1.03626 \mathrm{E}-14$ & $53.4+39.6(93)$ \\
\hline 77 & $2 s^{2} 2 p^{5} 5 d^{13} D^{0}$ & 2 & 1002.849 & 1002.942 & $1.03915 \mathrm{E}-14$ & $54.5+18.2(101)+18.1(35)$ \\
\hline 78 & $2 s^{2} 2 p^{5} 5 d^{13} F^{0}$ & 4 & 1004.21 & 1004.313 & $1.02377 E-14$ & 100 \\
\hline 79 & $2 s^{2} 2 p^{5} 5 d^{11} D^{0}$ & 2 & 1004.301 & 1004.401 & $1.01978 \mathrm{E}-14$ & $48.3+41(38)$ \\
\hline 80 & $2 s^{2} 2 p^{5} 5 d^{13} D^{0}$ & 3 & 1004.437 & 1004.532 & $1.02533 \mathrm{E}-14$ & $70.7+27(93)$ \\
\hline 81 & $2 s^{2} 2 p^{5} 5 d^{11} p^{0}$ & 1 & 1004.753 & 1004.833 & $2.0346 \mathrm{E}-15$ & $63.7+19.7(102)$ \\
\hline 82 & $2 s^{2} 2 p^{5} 5 f^{13} D^{e}$ & 1 & 1005.503 & 1004.963 & $6.74192 \mathrm{E}-15$ & 98.6 \\
\hline 83 & $2 s^{2} 2 p^{5} 5 f^{13} G^{e}$ & 4 & 1005.521 & 1004.996 & $6.85281 \mathrm{E}-15$ & $53.1+43.8(109)$ \\
\hline 84 & $2 s^{2} 2 p^{5} 5 f^{13} D^{e}$ & 2 & 1005.599 & 1005.61 & $6.76387 \mathrm{E}-15$ & $47.2+45.6(46)$ \\
\hline
\end{tabular}




\begin{tabular}{|c|c|c|c|c|c|c|}
\hline 85 & $2 s^{2} 2 p^{5} 5 f^{13} F^{e}$ & 3 & 1005.637 & 1005.63 & 6.81417E-15 & $50.7+22.7(114)+22.5(106)$ \\
\hline 86 & $2 s^{2} 2 p^{5} 4 p^{11} D^{e}$ & 2 & 1004.763 & 1005.704 & $1.78863 \mathrm{E}-14$ & $49.4+28.4(28)$ \\
\hline 87 & $2 s^{2} 2 p^{5} 5 f^{13} G^{e}$ & 4 & 1004.788 & 1005.744 & $1.75276 \mathrm{E}-14$ & $46.6+31.2(123)$ \\
\hline 88 & $2 s^{2} 2 p^{5} 5 f^{13} G^{e}$ & 5 & 1006.193 & 1006.302 & $6.78296 \mathrm{E}-15$ & 100 \\
\hline 89 & $2 s^{2} 2 p^{5} 5 f^{11} D^{e}$ & 2 & 1006.284 & 1006.385 & $6.85681 \mathrm{E}-15$ & $58.2+30.7(50)$ \\
\hline 90 & $2 s^{2} 2 p^{5} 5 f^{11} F^{e}$ & 3 & 1006.284 & 1006.391 & $6.99484 \mathrm{E}-15$ & $44.1+40.3(111)$ \\
\hline 91 & $2 s^{2} 2 p^{5} 5 f^{13} F^{e}$ & 4 & 1006.328 & 1006.435 & $7.03991 \mathrm{E}-15$ & $71.9+22.8(109)$ \\
\hline 92 & $2 s^{2} 2 p^{5} 5 g^{13} F^{0}$ & 3 & 1006.397 & 1006.495 & $1.21066 \mathrm{E}-14$ & 100 \\
\hline 93 & $2 s^{2} 2 p^{5} 5 g^{13} H^{0}$ & 5 & 1006.4 & 1006.498 & 1.20553E-14 & $52.6+45.7(57)$ \\
\hline 94 & $2 s^{2} 2 p^{5} 5 g^{13} G^{0}$ & 3 & 1006.45 & 1006.548 & $1.20888 \mathrm{E}-14$ & $54.3+36.5(130)$ \\
\hline 95 & $2 s^{2} 2 p^{5} 5 g^{13} G^{0}$ & 4 & 1006.468 & 1006.567 & 1.20659E-14 & $47.5+25.2(126)+25.1(129)$ \\
\hline 96 & $2 s^{2} 2 p^{5} 5 g^{11} F^{0}$ & 3 & 1006.809 & 1006.906 & 1.22703E-14 & $57.6+38.6(130)$ \\
\hline 97 & $2 s^{2} 2 p^{5} 5 g^{13} H^{0}$ & 6 & 1006.808 & 1006.907 & 1.22067E-14 & 100 \\
\hline 98 & $2 s^{2} 2 p^{5} 5 g^{11} G^{0}$ & 4 & 1006.86 & 1006.959 & $1.22371 \mathrm{E}-14$ & $41.6+39.4(131)+17.6(127)$ \\
\hline 99 & $2 s^{2} 2 p^{5} 5 g^{13} G^{0}$ & 5 & 1006.875 & 1006.973 & 1.22194E-14 & $71.6+21(57)$ \\
\hline 100 & $2 s^{1} 2 p^{6} 4 d^{13} F^{0}$ & 2 & 1009.514 & 1009.684 & $7.72721 \mathrm{E}-15$ & $74.5+20.2(47)$ \\
\hline 101 & $2 s^{2} 2 p^{5} 4 d^{13} D^{0}$ & 1 & 1010.205 & 1010.354 & 1.08509E-15 & $49.1+33.9(44)+16.2(49)$ \\
\hline 102 & $2 s^{1} 2 p^{5} 4 d^{13} p^{0}$ & 2 & 1012.559 & 1012.734 & 7.40903E-15 & $47.6+35.8(51)$ \\
\hline 103 & $2 s^{1} 2 p^{6} 4 d^{11} F^{0}$ & 3 & 1012.635 & 1012.809 & 7.44265E-15 & $44.2+33.4(41)+22.3(34)$ \\
\hline 104 & $2 s^{2} 2 p^{5} 4 f^{13} G^{e}$ & 3 & 1015.185 & 1015.37 & 3.60904E-15 & $73.4+19.1(56)$ \\
\hline 105 & $2 s^{2} 2 p^{5} 4 f^{13} D^{e}$ & 2 & 1015.255 & 1015.44 & 3.55303E-15 & $44.6+33.3(66)+22.1(29)$ \\
\hline 106 & $2 s^{2} 2 p^{5} 4 f^{13} D^{e}$ & 3 & 1016.545 & 1016.723 & 3.68986E-15 & $54.3+29.8(64)$ \\
\hline 107 & $2 s^{2} 2 p^{5} 4 f^{11} G^{e}$ & 4 & 1016.538 & 1016.73 & $3.71828 \mathrm{E}-15$ & $41.6+33.3(59)+25.1(62)$ \\
\hline 108 & $2 s^{1} 2 p^{6} 4 s^{13} s^{e}$ & 1 & 1024.648 & 1024.385 & $9.98655 \mathrm{E}-15$ & 99.8 \\
\hline 109 & $2 s^{1} 2 p^{6} 4 s^{11} s^{e}$ & 0 & 1025.487 & 1025.184 & 1.00719E-14 & 100 \\
\hline 110 & $2 s^{1} 2 p^{6} 4 p^{13} p^{0}$ & 0 & 1029.366 & 1029.042 & $8.71687 \mathrm{E}-15$ & 100 \\
\hline 111 & $2 s^{1} 2 p^{6} 4 p^{13} p^{0}$ & 1 & 1029.393 & 1029.069 & $3.7232 \mathrm{E}-15$ & $67.4+32.5(183)$ \\
\hline 112 & $2 s^{1} 2 p^{6} 4 p^{13} p^{0}$ & 2 & 1041.608 & 1041.312 & 1.22735E-14 & 99.8 \\
\hline 113 & $2 s^{1} 2 p^{6} 4 p^{11} p^{0}$ & 1 & 1041.771 & 1041.471 & $2.99884 \mathrm{E}-15$ & $67.6+32.5(171)$ \\
\hline 114 & $2 s^{1} 2 p^{6} 4 d^{13} D^{e}$ & 1 & 1046.346 & 1046.004 & $6.43442 \mathrm{E}-15$ & 100 \\
\hline 115 & $2 s^{1} 2 p^{6} 4 d^{13} D^{e}$ & 2 & 1046.481 & 1046.141 & 6.38217E-15 & $67.9+32(172)$ \\
\hline 116 & $2 s^{1} 2 p^{6} 4 d^{13} D^{e}$ & 3 & 1049.274 & 1048.949 & $6.21943 \mathrm{E}-15$ & 99.8 \\
\hline 117 & $2 s^{1} 2 p^{6} 4 d^{11} D^{e}$ & 2 & 1049.681 & 1049.344 & $6.1474 \mathrm{E}-15$ & $67.9+32(177)$ \\
\hline 118 & $2 s^{2} 2 p^{5} 6 s^{13} p^{0}$ & 2 & 1051.706 & 1051.54 & $2.38246 \mathrm{E}-14$ & 100 \\
\hline 119 & $2 s^{2} 2 p^{5} 6 s^{11} p^{0}$ & 1 & 1051.755 & 1051.549 & $1.70684 \mathrm{E}-14$ & $66.6+33.4(73)$ \\
\hline 120 & $2 s^{2} 2 p^{5} 6 p^{13} p^{e}$ & 1 & 1053.042 & 1051.777 & 1.99187E-14 & $49.8+30.7(108)$ \\
\hline 121 & $2 s^{2} 2 p^{5} 6 p^{13} D^{e}$ & 2 & 1053.053 & 1051.822 & $1.98729 \mathrm{E}-14$ & $50+33.4(83)+16.6(100)$ \\
\hline 122 & $2 s^{1} 2 p^{6} 4 f^{13} F^{0}$ & 2 & 1051.866 & 1052.848 & $3.28496 \mathrm{E}-15$ & 100 \\
\hline 123 & $2 s^{1} 2 p^{6} 4 f^{13} F^{0}$ & 3 & 1051.845 & 1052.922 & 3.28959E-15 & $59.9+40.1(184)$ \\
\hline 124 & $2 s^{1} 2 p^{6} 4 f^{13} F^{0}$ & 4 & 1053.153 & 1053.096 & 3.37137E-15 & 100 \\
\hline 125 & $2 s^{1} 2 p^{6} 4 f^{11} F^{0}$ & 3 & 1053.241 & 1053.123 & 3.38436E-15 & $59.9+40.1(182)$ \\
\hline 126 & $2 s^{2} 2 p^{5} 6 p^{11} p^{e}$ & 1 & 1056.56 & 1056.665 & $2.83002 \mathrm{E}-14$ & $56.3+36(108)$ \\
\hline 127 & $2 s^{2} 2 p^{5} 6 p^{13} D^{e}$ & 3 & 1056.554 & 1056.666 & $2.89775 \mathrm{E}-14$ & 99.8 \\
\hline 128 & $2 s^{2} 2 p^{5} 6 p^{13} p^{e}$ & 2 & 1056.647 & 1056.745 & 2.87797E-14 & $66.7+33.2(100)$ \\
\hline 129 & $2 s^{2} 2 p^{5} 6 p^{11} s^{e}$ & 0 & 1057.014 & 1057.075 & 2.79655E-14 & $66.4+33.5(107)$ \\
\hline 130 & $2 s^{2} 2 p^{5} 6 d^{13} p^{0}$ & 0 & 1057.823 & 1057.919 & 1.53827E-14 & 100 \\
\hline 131 & $2 s^{2} 2 p^{5} 6 d^{13} p^{0}$ & 1 & 1057.892 & 1058.011 & 1.32699E-14 & $62.3+34.9(146)$ \\
\hline 132 & $2 s^{2} 2 p^{5} 6 d^{13} F^{0}$ & 3 & 1057.891 & 1058.029 & $1.53689 \mathrm{E}-14$ & $53.4+39.6(143)$ \\
\hline 133 & $2 s^{2} 2 p^{5} 6 d^{13} D^{0}$ & 2 & 1057.95 & 1058.066 & 1.53425E-14 & $54.3+18.5(147)+18.2(142)$ \\
\hline 134 & $2 s^{2} 2 p^{5} 6 d^{13} F^{0}$ & 4 & 1058.743 & 1058.903 & $1.53841 \mathrm{E}-14$ & 100 \\
\hline 135 & $2 s^{2} 2 p^{5} 6 d^{11} D^{0}$ & 2 & 1058.796 & 1058.938 & $1.53102 \mathrm{E}-14$ & $48.2+41.1(149)$ \\
\hline 136 & $2 s^{2} 2 p^{5} 6 d^{13} D^{\circ}$ & 3 & 1058.867 & 1059.007 & $1.5321 \mathrm{E}-14$ & $70.9+27(143)$ \\
\hline 137 & $2 s^{2} 2 p^{5} 6 d^{11} p^{0}$ & 1 & 1059.065 & 1059.198 & $2.9064 \mathrm{E}-15$ & $63.7+21.1(148)$ \\
\hline 138 & $2 s^{2} 2 p^{5} 7 s^{13} p^{0}$ & 2 & 1087.093 & 1087.216 & 3.25733E-14 & 100 \\
\hline 139 & $2 s^{2} 2 p^{5} 7 s^{11} p^{0}$ & 1 & 1087.126 & 1087.245 & $2.49368 \mathrm{E}-14$ & $66.6+33.3(152)$ \\
\hline 140 & $2 s^{2} 2 p^{5} 7 p^{13} p^{e}$ & 1 & 1087.928 & 1088.04 & $2.75105 \mathrm{E}-14$ & $50+30.8(168)$ \\
\hline 141 & $2 s^{2} 2 p^{5} 7 p^{13} D^{e}$ & 2 & 1087.933 & 1088.054 & $2.74228 \mathrm{E}-14$ & $50+33.4(159)+16.6(161)$ \\
\hline 142 & $2 s^{2} 2 p^{5} 7 p^{13} D^{e}$ & 3 & 1090.119 & 1090.267 & 3.84874E-14 & 100 \\
\hline 143 & $2 s^{2} 2 p^{5} 7 p^{11} p^{e}$ & 1 & 1090.124 & 1090.267 & 3.69642E-14 & $56.4+35.9(168)$ \\
\hline 144 & $2 s^{2} 2 p^{5} 7 p^{13} p^{e}$ & 2 & 1090.178 & 1090.316 & 3.79865E-14 & $66.7+33.2(159)$ \\
\hline
\end{tabular}




\begin{tabular}{|c|c|c|c|c|c|c|}
\hline 145 & $2 s^{2} 2 p^{5} 7 p^{11} s^{e}$ & 0 & 1090.396 & 1090.51 & $3.57154 \mathrm{E}-14$ & $66.4+33.4(176)$ \\
\hline 146 & $2 s^{2} 2 p^{5} 7 d^{13} p^{0}$ & 0 & 1090.909 & 1091.049 & $2.2243 \mathrm{E}-14$ & 100 \\
\hline 147 & $2 s^{2} 2 p^{5} 7 d^{13} p^{0}$ & 1 & 1090.951 & 1091.105 & $1.93798 \mathrm{E}-14$ & $62.4+34.9(202)$ \\
\hline 148 & $2 s^{2} 2 p^{5} 7 d^{13} F^{0}$ & 3 & 1090.95 & 1091.115 & $2.21394 \mathrm{E}-14$ & $53.4+39.7(185)$ \\
\hline 149 & $2 s^{2} 2 p^{5} 7 d^{13} D^{0}$ & 2 & 1090.986 & 1091.137 & $2.20198 \mathrm{E}-14$ & $54.3+18.5(191)+18.2(192)$ \\
\hline 150 & $2 s^{2} 2 p^{5} 7 d^{13} F^{0}$ & 4 & 1091.488 & 1091.666 & $2.25708 \mathrm{E}-14$ & 100 \\
\hline 151 & $2 s^{2} 2 p^{5} 7 d^{11} D^{0}$ & 2 & 1091.52 & 1091.687 & $2.23373 \mathrm{E}-14$ & $48.2+41.1(194)$ \\
\hline 152 & $2 s^{2} 2 p^{5} 7 d^{13} D^{0}$ & 3 & 1091.564 & 1091.729 & $2.22492 \mathrm{E}-14$ & $70.9+27(185)$ \\
\hline 153 & $2 s^{2} 2 p^{5} 7 d^{11} p^{0}$ & 1 & 1091.684 & 1091.842 & 4.86779E-15 & $63.8+21(206)$ \\
\hline 154 & $2 s^{2} 2 p^{5} 5 s^{13} p^{0}$ & 0 & 1100.412 & 1092.106 & 1.67625E-14 & 100 \\
\hline 155 & $2 s^{2} 2 p^{5} 5 s^{13} p^{0}$ & 1 & 1100.443 & 1092.13 & 1.43825E-14 & $66.6+33.4(71)$ \\
\hline 156 & $2 s^{2} 2 p^{5} 5 p^{13} D^{e}$ & 1 & 1102.702 & 1102.913 & $1.38953 \mathrm{E}-14$ & $74+22.3(85)$ \\
\hline 157 & $2 s^{2} 2 p^{5} 5 p^{13} p^{e}$ & 0 & 1103.281 & 1103.469 & $1.4156 \mathrm{E}-14$ & $66.1+33.8(87)$ \\
\hline 158 & $2 s^{2} 2 p^{5} 5 p^{13} s^{e}$ & 1 & 1108.935 & 1109.156 & 2.11005E-14 & $50.1+29.5(81)$ \\
\hline 159 & $2 s^{2} 2 p^{5} 5 p^{11} D^{e}$ & 2 & 1108.947 & 1109.169 & $2.08835 \mathrm{E}-14$ & $50+33.4(79)+16.6(72)$ \\
\hline 160 & $2 s^{2} 2 p^{5} 5 d^{13} F^{0}$ & 2 & 1111.254 & 1111.465 & 1.03893E-14 & $74.6+20.1(35)$ \\
\hline 161 & $2 s^{2} 2 p^{5} 5 d^{13} D^{0}$ & 1 & 1111.574 & 1111.771 & $2.30698 \mathrm{E}-15$ & $49.8+33.6(99)+16.5(102)$ \\
\hline 162 & $2 s^{2} 2 p^{5} 5 d^{13} p^{0}$ & 2 & 1112.822 & 1113.036 & $1.021 \mathrm{E}-14$ & $47.9+35.6(97)$ \\
\hline 163 & $2 s^{2} 2 p^{5} 5 d^{11} F^{0}$ & 3 & 1112.858 & 1113.071 & $1.02483 \mathrm{E}-14$ & $44.4+33.4(93)+22.3(103)$ \\
\hline 164 & $2 s^{2} 2 p^{5} 5 f^{13} G^{e}$ & 3 & 1114.116 & 1114.337 & $6.83421 \mathrm{E}-15$ & $73.4+19.1(106)$ \\
\hline 165 & $2 s^{2} 2 p^{5} 5 f^{13} F^{e}$ & 2 & 1114.16 & 1114.38 & 6.69337E-15 & $44.5+33.3(48)+22.2(50)$ \\
\hline 166 & $2 s^{2} 2 p^{5} 5 f^{13} D^{e}$ & 3 & 1114.815 & 1115.035 & $6.99436 \mathrm{E}-15$ & $54.3+29.8(104)$ \\
\hline 167 & $2 s^{2} 2 p^{5} 5 f^{11} G^{e}$ & 4 & 1114.814 & 1115.036 & 7.05689E-15 & $41.6+33.3(109)+25(112)$ \\
\hline 168 & $2 s^{2} 2 p^{5} 5 g^{13} H^{0}$ & 4 & 1114.995 & 1115.205 & $1.20581 \mathrm{E}-14$ & $72.4+18.5(129)$ \\
\hline 169 & $2 s^{2} 2 p^{5} 5 g^{13} F^{0}$ & 3 & 1115.003 & 1115.214 & $1.20224 \mathrm{E}-14$ & $41.7+33.3(135)+25(130)$ \\
\hline 170 & $2 s^{2} 2 p^{5} 5 g^{11} H^{0}$ & 5 & 1115.404 & 1115.614 & 1.22139E-14 & $39.9+33.3(57)+26.7(60)$ \\
\hline 171 & $2 s^{2} 2 p^{5} 5 g^{13} F^{0}$ & 4 & 1115.41 & 1115.621 & 1.21712E-14 & $57.6+26.6(127)$ \\
\hline 172 & $2 s^{1} 2 p^{6} 5 s^{13} s^{e}$ & 1 & 1137.113 & 1136.873 & 1.14169E-14 & 100 \\
\hline 173 & $2 s^{1} 2 p^{6} 5 s^{11} s^{e}$ & 0 & 1137.514 & 1137.245 & 1.1377E-14 & 100 \\
\hline 174 & $2 s^{1} 2 p^{6} 5 p^{13} p^{0}$ & 0 & 1139.491 & 1139.215 & $1.00681 \mathrm{E}-14$ & 100 \\
\hline 175 & $2 s^{1} 2 p^{6} 5 p^{13} p^{0}$ & 1 & 1139.503 & 1139.226 & $5.43028 \mathrm{E}-15$ & $67.4+32.5(190)$ \\
\hline 176 & $2 s^{1} 2 p^{6} 5 p^{13} p^{0}$ & 2 & 1145.66 & 1145.399 & 1.3317E-14 & 100 \\
\hline 177 & $2 s^{1} 2 p^{6} 5 p^{11} p^{0}$ & 1 & 1145.734 & 1145.469 & 4.61479E-15 & $67.4+32.5(195)$ \\
\hline 178 & $2 s^{1} 2 p^{6} 5 d^{13} D^{e}$ & 1 & 1147.977 & 1147.707 & $8.02283 \mathrm{E}-15$ & 100 \\
\hline 179 & $2 s^{1} 2 p^{6} 5 d^{13} D^{e}$ & 2 & 1148.047 & 1147.773 & $7.95701 \mathrm{E}-15$ & $67.4+32.5(199)$ \\
\hline 180 & $2 s^{1} 2 p^{6} 5 d^{13} D^{e}$ & 3 & 1149.49 & 1149.227 & $7.95418 \mathrm{E}-15$ & 100 \\
\hline 181 & $2 s^{1} 2 p^{6} 5 d^{11} D^{e}$ & 2 & 1149.682 & 1149.407 & 7.86477E-15 & $67.4+32.5(207)$ \\
\hline 182 & $2 s^{1} 2 p^{6} 5 f^{13} F^{0}$ & 2 & 1150.76 & 1150.51 & $5.75814 \mathrm{E}-15$ & 100 \\
\hline 183 & $2 s^{1} 2 p^{6} 5 f^{13} F^{0}$ & 3 & 1150.759 & 1150.514 & $5.74263 \mathrm{E}-15$ & $60.8+39.1(205)$ \\
\hline 184 & $2 s^{1} 2 p^{6} 5 f^{13} F^{0}$ & 4 & 1151.426 & 1151.178 & 5.89964E-15 & 100 \\
\hline 185 & $2 s^{1} 2 p^{6} 5 f^{11} F^{0}$ & 3 & 1151.475 & 1151.228 & $5.93046 \mathrm{E}-15$ & $60.8+39.1(205)$ \\
\hline 186 & $2 s^{1} 2 p^{6} 5 g^{13} G^{e}$ & 3 & 1151.617 & 1151.351 & $9.25868 \mathrm{E}-15$ & 100 \\
\hline 187 & $2 s^{1} 2 p^{6} 5 g^{13} G^{e}$ & 4 & 1151.604 & 1151.367 & $9.20854 \mathrm{E}-15$ & $55.8+44.2(74)$ \\
\hline 188 & $2 s^{1} 2 p^{6} 5 g^{13} G^{e}$ & 5 & 1152.012 & 1151.759 & $9.29581 \mathrm{E}-15$ & 100 \\
\hline 189 & $2 s^{1} 2 p^{6} 5 g^{11} G^{e}$ & 4 & 1152.024 & 1151.775 & $9.35053 \mathrm{E}-15$ & $55.8+44.2(67)$ \\
\hline 190 & $2 s^{2} 2 p^{5} 6 s^{13} p^{0}$ & 0 & 1160.21 & 1160.482 & 2.36377E-14 & 100 \\
\hline 191 & $2 s^{2} 2 p^{5} 6 s^{13} p^{0}$ & 1 & 1160.226 & 1160.509 & 2.07965E-14 & $66.6+33.4(84)$ \\
\hline 192 & $2 s^{2} 2 p^{5} 6 p^{13} D^{e}$ & 1 & 1161.514 & 1161.798 & $1.97575 \mathrm{E}-14$ & $74+22.3(113)$ \\
\hline 193 & $2 s^{2} 2 p^{5} 6 p^{13} p^{e}$ & 0 & 1161.817 & 1162.072 & $1.97973 \mathrm{E}-14$ & $66.4+33.5(110)$ \\
\hline 194 & $2 s^{2} 2 p^{5} 6 p^{13} s^{e}$ & 1 & 1165.08 & 1165.383 & 2.88513E-14 & $50+29.6(108)$ \\
\hline 195 & $2 s^{2} 2 p^{5} 6 p^{11} D^{e}$ & 2 & 1165.084 & 1165.406 & $2.86028 \mathrm{E}-14$ & $50+33.3(100)+16.6(96)$ \\
\hline 196 & $2 s^{2} 2 p^{5} 6 d^{13} F^{0}$ & 2 & 1166.387 & 1166.731 & $1.5421 \mathrm{E}-14$ & $74.6+20(147)$ \\
\hline 197 & $2 s^{2} 2 p^{5} 6 d^{13} D^{0}$ & 1 & 1166.561 & 1166.903 & 4.09683E-15 & $49.8+33.5(153)+16.6(148)$ \\
\hline 198 & $2 s^{2} 2 p^{5} 6 d^{13} p^{0}$ & 2 & 1167.296 & 1167.649 & $1.5344 \mathrm{E}-14$ & $47.9+35.6(144)$ \\
\hline 199 & $2 s^{2} 2 p^{5} 6 d^{11} F^{0}$ & 3 & 1167.315 & 1167.675 & 1.53799E-14 & $44.4+33.3(143)+22.3(145)$ \\
\hline 200 & $2 s^{2} 2 p^{5} 7 s^{13} p^{0}$ & 0 & 1195.609 & 1195.929 & 3.27248E-14 & 100 \\
\hline 201 & $2 s^{2} 2 p^{5} 7 s^{13} p^{0}$ & 1 & 1195.618 & 1195.95 & $2.94384 \mathrm{E}-14$ & $66.6+33.3(157)$ \\
\hline 202 & $2 s^{2} 2 p^{5} 7 p^{13} D^{e}$ & 1 & 1196.421 & 1196.608 & $2.74488 \mathrm{E}-14$ & $74.1+22.3(165)$ \\
\hline 203 & $2 s^{2} 2 p^{5} 7 p^{13} p^{e}$ & 0 & 1196.606 & 1196.768 & 2.70753E-14 & $66.6+33.5(180)$ \\
\hline 204 & $2 s^{2} 2 p^{5} 7 p^{13} s^{e}$ & 1 & 1198.647 & 1196.807 & 3.84103E-14 & $50+29.6(168)$ \\
\hline 205 & $2 s^{2} 2 p^{5} 7 p^{11} D^{e}$ & 2 & 1198.65 & 1196.934 & 3.82344E-14 & $50+33.3(159)+16.6(161)$ \\
\hline 206 & $2 s^{2} 2 p^{5} 7 d^{13} F^{0}$ & 2 & 1199.458 & 1197.972 & 2.23397E-14 & $74.6+20(191)$ \\
\hline
\end{tabular}




\begin{tabular}{lllllll}
\hline 207 & $2 s^{2} 2 p^{5} 7 d^{13} D^{\circ}$ & 1 & 1199.565 & 1197.985 & $6.58752 \mathrm{E}-15$ & $50+33.4(204)+16.6(206)$ \\
208 & $2 s^{2} 2 p^{5} 7 d^{13} p^{0}$ & 2 & 1200.031 & 1199.002 & $2.24744 \mathrm{E}-14$ & $48+35.6(196)$ \\
209 & $2 s^{2} 2 p^{5} 7 d^{11} F^{0}$ & 3 & 1200.042 & 1199.008 & $2.25037 E-14$ & $44.4+33.3(185)+22.3(187)$ \\
\hline
\end{tabular}

Table 5: Transition wavelength (in Å), transition probability in $\mathrm{s}^{-1}$, oscillator strength (dimensionless), line strength (length form) for E1 transition using MCDF for Ne-like $\mathrm{Hf}$

\begin{tabular}{|c|c|c|c|c|c|c|}
\hline $\mathrm{i}$ & $j$ & $\lambda$ (in $\AA$ ) & $A_{j i}\left(s^{-1}\right)$ & $f_{i j}$ & $\mathrm{~S}_{\mathrm{ij}}(\mathrm{au})$ & Vel./len \\
\hline $2 s^{2} 2 p^{6}{ }^{1} S_{0}$ & $2 s^{2} 2 p^{5} 3 s^{1} P_{1}$ & $1.58 \mathrm{E}+00$ & $1.35 \mathrm{E}+14$ & $1.52 \mathrm{E}-01$ & $7.89 \mathrm{E}-04$ & $9.90 \mathrm{E}-01$ \\
\hline $2 s^{2} 2 p^{6}{ }^{1} S_{0}$ & $2 s^{2} 2 p^{5} 3 d^{3} P_{1}$ & $1.46 \mathrm{E}+00$ & $6.37 E+13$ & $6.14 \mathrm{E}-02$ & 2.96E-04 & $1.00 \mathrm{E}+00$ \\
\hline $2 s^{2} 2 p^{6}{ }^{1} S_{0}$ & $2 s^{2} 2 p^{5} 3 d^{1} P_{1}$ & $1.44 \mathrm{E}+00$ & $2.48 \mathrm{E}+15$ & $2.32 \mathrm{E}+00$ & 1.10E-02 & $1.00 \mathrm{E}+00$ \\
\hline $2 s^{2} 2 p^{6}{ }^{1} S_{0}$ & $2 s^{2} 2 p^{5} 3 s^{3} P_{1}$ & $1.37 \mathrm{E}+00$ & $3.32 \mathrm{E}+13$ & $2.80 \mathrm{E}-02$ & $1.26 \mathrm{E}-04$ & $9.70 \mathrm{E}-01$ \\
\hline $2 s^{2} 2 p^{6}{ }^{1} s_{0}$ & $2 s 2 p^{6} 3 p^{3} P_{1}$ & $1.28 \mathrm{E}+00$ & $9.93 E+14$ & $7.34 \mathrm{E}-01$ & 3.10E-03 & $1.00 \mathrm{E}+00$ \\
\hline $2 s^{2} 2 p^{6}{ }^{1} S_{0}$ & $2 s^{2} 2 p^{5} 3 d^{3} D_{1}$ & $1.28 \mathrm{E}+00$ & $8.85 E+14$ & $6.47 E-01$ & $2.72 \mathrm{E}-03$ & $1.00 \mathrm{E}+00$ \\
\hline $2 s^{2} 2 p^{6}{ }^{1} s_{0}$ & $2 s 2 p^{6} 3 p{ }^{1} P_{1}$ & $1.24 \mathrm{E}+00$ & $4.00 \mathrm{E}+14$ & $2.75 \mathrm{E}-01$ & $1.12 \mathrm{E}-03$ & $1.00 \mathrm{E}+00$ \\
\hline $2 s^{2} 2 p^{6}{ }^{1} S_{0}$ & $2 s^{2} 2 p^{5} 4 s^{1} P_{1}$ & $1.13 \mathrm{E}+00$ & 4.77E+13 & $2.75 \mathrm{E}-02$ & $1.02 \mathrm{E}-04$ & $9.40 \mathrm{E}-01$ \\
\hline $2 s^{2} 2 p^{6}{ }^{1} s_{0}$ & $2 s^{2} 2 p^{5} 4 d^{3} P_{1}$ & $1.11 \mathrm{E}+00$ & $2.60 \mathrm{E}+13$ & $1.43 \mathrm{E}-02$ & $5.22 \mathrm{E}-05$ & $9.90 \mathrm{E}-01$ \\
\hline $2 s^{2} 2 p^{6}{ }^{1} s_{0}$ & $2 s^{2} 2 p^{5} 4 d^{1} P_{1}$ & $1.10 \mathrm{E}+00$ & $8.98 \mathrm{E}+14$ & 4.90E-01 & $1.78 \mathrm{E}-03$ & $9.90 \mathrm{E}-01$ \\
\hline $2 s^{2} 2 p^{6}{ }^{1} S_{0}$ & $2 s^{2} 2 p^{5} 4 s^{3} P_{1}$ & $1.02 \mathrm{E}+00$ & $1.73 \mathrm{E}+13$ & $8.07 E-03$ & $2.71 \mathrm{E}-05$ & $9.10 \mathrm{E}-01$ \\
\hline $2 s^{2} 2 p^{6}{ }^{1} S_{0}$ & $2 s^{2} 2 p^{5} 5 s^{1} P_{1}$ & $1.01 \mathrm{E}+00$ & $3.02 \mathrm{E}+13$ & $1.37 \mathrm{E}-02$ & 4.54E-05 & $9.00 \mathrm{E}-01$ \\
\hline $2 s^{2} 2 p^{6}{ }^{1} S_{0}$ & $2 s^{2} 2 p^{5} 4 d^{3} D_{1}$ & 9.97E-01 & $4.51 \mathrm{E}+14$ & $2.02 \mathrm{E}-01$ & $6.61 \mathrm{E}-04$ & $9.90 \mathrm{E}-01$ \\
\hline $2 s^{2} 2 p^{6}{ }^{1} s_{0}$ & $2 s^{2} 2 p^{5} 5 d^{3} P_{1}$ & $9.95 \mathrm{E}-01$ & $9.74 \mathrm{E}+12$ & $4.33 \mathrm{E}-03$ & $1.42 \mathrm{E}-05$ & $9.80 \mathrm{E}-01$ \\
\hline $2 s^{2} 2 p^{6}{ }^{1} S_{0}$ & $2 s^{2} 2 p^{5} 5 d^{1} P_{1}$ & 9.93E-01 & $5.46 \mathrm{E}+14$ & $2.42 \mathrm{E}-01$ & 7.91E-04 & $9.80 \mathrm{E}-01$ \\
\hline $2 s^{2} 2 p^{6}{ }^{1} s_{0}$ & $2 s 2 p^{6} 4 p{ }^{3} P_{1}$ & 9.76E-01 & $1.26 \mathrm{E}+14$ & $5.39 \mathrm{E}-02$ & $1.73 \mathrm{E}-04$ & $9.80 \mathrm{E}-01$ \\
\hline $2 s^{2} 2 p^{6}{ }^{1} S_{0}$ & $2 s 2 p^{6} 4 p{ }^{1} P_{1}$ & $9.66 \mathrm{E}-01$ & $2.12 \mathrm{E}+14$ & $8.90 \mathrm{E}-02$ & 2.83E-04 & $9.80 \mathrm{E}-01$ \\
\hline $2 s^{2} 2 p^{6}{ }^{1} S_{0}$ & $2 s^{2} 2 p^{5} 6 s \quad{ }^{1} P_{1}$ & $9.48 \mathrm{E}-01$ & $1.32 \mathrm{E}+13$ & $5.35 \mathrm{E}-03$ & $1.67 \mathrm{E}-05$ & $8.20 \mathrm{E}-01$ \\
\hline $2 s^{2} 2 p^{6}{ }^{1} S_{0}$ & $2 s^{2} 2 p^{5} 6 d^{3} P_{1}$ & $9.43 \mathrm{E}-01$ & $7.74 \mathrm{E}+12$ & $3.10 \mathrm{E}-03$ & $9.61 \mathrm{E}-06$ & $9.70 \mathrm{E}-01$ \\
\hline $2 s^{2} 2 p^{6}{ }^{1} S_{0}$ & $2 s^{2} 2 p^{5} 6 d{ }^{1} P_{1}$ & $9.42 \mathrm{E}-01$ & $2.35 E+14$ & $9.37 \mathrm{E}-02$ & $2.91 \mathrm{E}-04$ & $9.60 \mathrm{E}-01$ \\
\hline $2 s^{2} 2 p^{6}{ }^{1} S_{0}$ & $2 s^{2} 2 p^{5} 7 s^{1} P_{1}$ & $9.18 \mathrm{E}-01$ & $7.09 \mathrm{E}+12$ & $2.68 \mathrm{E}-03$ & $8.11 \mathrm{E}-06$ & $1.00 \mathrm{E}+00$ \\
\hline $2 s^{2} 2 p^{6}{ }^{1} S_{0}$ & $2 s^{2} 2 p^{5} 7 d^{3} P_{1}$ & $9.15 \mathrm{E}-01$ & $9.34 \mathrm{E}+10$ & $3.51 \mathrm{E}-05$ & $1.06 \mathrm{E}-07$ & $2.10 \mathrm{E}+00$ \\
\hline $2 s^{2} 2 p^{6}{ }^{1} S_{0}$ & $2 s^{2} 2 p^{5} 7 d^{1} P_{1}$ & $9.14 \mathrm{E}-01$ & $1.24 \mathrm{E}+14$ & $4.67 \mathrm{E}-02$ & $1.41 \mathrm{E}-04$ & $1.00 \mathrm{E}+00$ \\
\hline $2 s^{2} 2 p^{6}{ }^{1} S_{0}$ & $2 s^{2} 2 p^{5} 5 s^{3} P_{1}$ & $9.14 \mathrm{E}-01$ & $1.87 \mathrm{E}+13$ & $7.02 \mathrm{E}-03$ & $2.11 \mathrm{E}-05$ & $9.10 \mathrm{E}-01$ \\
\hline $2 s^{2} 2 p^{6}{ }^{1} S_{0}$ & $2 s^{2} 2 p^{5} 5 d^{3} D_{1}$ & $9.06 \mathrm{E}-01$ & $2.84 \mathrm{E}+14$ & $1.05 \mathrm{E}-01$ & 3.12E-04 & $9.80 \mathrm{E}-01$ \\
\hline $2 s^{2} 2 p^{6}{ }^{1} S_{0}$ & $2 s 2 p^{6} 5 p^{3} P_{1}$ & $8.82 \mathrm{E}-01$ & $6.94 \mathrm{E}+13$ & $2.43 \mathrm{E}-02$ & $7.05 \mathrm{E}-05$ & $9.80 \mathrm{E}-01$ \\
\hline $2 s^{2} 2 p^{6}{ }^{1} S_{0}$ & $2 s 2 p^{6} 5 p{ }^{1} P_{1}$ & $8.78 \mathrm{E}-01$ & $1.19 \mathrm{E}+14$ & $4.12 \mathrm{E}-02$ & 1.19E-04 & $9.60 \mathrm{E}-01$ \\
\hline $2 s^{2} 2 p^{6}{ }^{1} S_{0}$ & $2 s^{2} 2 p^{5} 6 s^{3} P_{1}$ & 8.67E-01 & $4.54 \mathrm{E}+12$ & $1.54 \mathrm{E}-03$ & $4.39 \mathrm{E}-06$ & 7.40E-01 \\
\hline $2 s^{2} 2 p^{6}{ }^{1} s_{0}$ & $2 s^{2} 2 p^{5} 6 d^{3} D_{1}$ & 8.63E-01 & $1.48 \mathrm{E}+14$ & 4.96E-02 & $1.41 \mathrm{E}-04$ & $9.60 \mathrm{E}-01$ \\
\hline $2 s^{2} 2 p^{6}{ }^{1} S_{0}$ & $2 s^{2} 2 p^{5} 7 s^{3} P_{1}$ & $8.42 \mathrm{E}-01$ & $2.46 \mathrm{E}+12$ & $7.85 \mathrm{E}-04$ & 2.17E-06 & $9.30 \mathrm{E}-01$ \\
\hline $2 s^{2} 2 p^{6}{ }^{1} S_{0}$ & $2 s^{2} 2 p^{5} 7 d^{3} D_{1}$ & $8.39 \mathrm{E}-01$ & $8.71 \mathrm{E}+13$ & $2.76 \mathrm{E}-02$ & $7.61 \mathrm{E}-05$ & $1.00 \mathrm{E}+00$ \\
\hline
\end{tabular}

Table 6: Transition wavelength (in Å), transition probability in $\mathrm{s}^{-1}$, oscillator strength (dimensionless), line strength (length form) for E1 transition using MCDF for Ne-like Ta

\begin{tabular}{llccccc}
\hline $\mathrm{i}$ & $\mathrm{j}$ & $\lambda$ (in $\AA$ ) & $\mathrm{A}_{\mathrm{j}}\left(\mathrm{s}^{-1}\right)$ & $\mathrm{f}_{\mathrm{ij}}$ & $\mathrm{s}_{\mathrm{ij}}(\mathrm{au})$ & \multicolumn{1}{c}{ Vel./len } \\
\hline $2 \mathrm{~s}^{2} 2 \mathrm{p}^{61} \mathrm{~s}_{0}$ & $2 \mathrm{~s}^{2} 2 \mathrm{p}^{5} 3 \mathrm{~s}^{1} \mathrm{P}_{1}$ & $1.54 \mathrm{E}+00$ & $1.44 \mathrm{E}+14$ & $1.53 \mathrm{E}-01$ & $7.74 \mathrm{E}-04$ & $9.90 \mathrm{E}-01$ \\
$2 \mathrm{~s}^{2} 2 \mathrm{p}^{61} \mathrm{~s}_{0}$ & $2 \mathrm{~s}^{2} 2 \mathrm{p}^{5} 3 \mathrm{~d}^{3} \mathrm{P}_{1}$ & $1.42 \mathrm{E}+00$ & $7.22 \mathrm{E}+13$ & $6.55 \mathrm{E}-02$ & $3.06 \mathrm{E}-04$ & $1.00 \mathrm{E}+00$ \\
$2 \mathrm{~s}^{2} 2 \mathrm{p}^{61} \mathrm{~s}_{0}$ & $2 \mathrm{~s}^{2} 2 \mathrm{p}^{5} 3 \mathrm{~d}^{1} \mathrm{P}_{1}$ & $1.40 \mathrm{E}+00$ & $2.63 \mathrm{E}+15$ & $2.32 \mathrm{E}+00$ & $1.07 \mathrm{E}-02$ & $1.00 \mathrm{E}+00$ \\
\hline
\end{tabular}




\begin{tabular}{|c|c|c|c|c|c|c|}
\hline $2 s^{2} 2 p^{61} s_{0}$ & $2 s^{2} 2 p^{5} 3 s^{3} P_{1}$ & $1.32 \mathrm{E}+00$ & $3.61 \mathrm{E}+13$ & $2.84 \mathrm{E}-02$ & $1.24 \mathrm{E}-04$ & $9.70 \mathrm{E}-01$ \\
\hline $2 s^{2} 2 p^{61} s_{0}$ & $2 s 2 p^{6} 3 p^{3} P_{1}$ & $1.24 \mathrm{E}+00$ & $8.71 \mathrm{E}+14$ & $6.03 \mathrm{E}-01$ & $2.46 \mathrm{E}-03$ & $1.00 \mathrm{E}+00$ \\
\hline $2 s^{2} 2 p^{61} s_{0}$ & $2 s^{2} 2 p^{5} 3 d^{3} D_{1}$ & $1.23 \mathrm{E}+00$ & $1.13 \mathrm{E}+15$ & 7.70E-01 & $3.13 \mathrm{E}-03$ & $1.00 \mathrm{E}+00$ \\
\hline $2 s^{2} 2 p^{61} s_{0}$ & $2 \mathrm{~s} 2 \mathrm{p}^{6} 3 \mathrm{p}^{1} \mathrm{P}_{1}$ & $1.20 \mathrm{E}+00$ & $4.22 \mathrm{E}+14$ & $2.72 \mathrm{E}-01$ & $1.07 \mathrm{E}-03$ & $1.00 E+00$ \\
\hline $2 s^{2} 2 p^{61} s_{0}$ & $2 s^{2} 2 p^{5} 4 s^{1} \mathrm{P}_{1}$ & $1.10 \mathrm{E}+00$ & $5.10 \mathrm{E}+13$ & $2.77 \mathrm{E}-02$ & $1.00 \mathrm{E}-04$ & $9.40 \mathrm{E}-01$ \\
\hline $2 s^{2} 2 p^{61} s_{0}$ & $2 s^{2} 2 p^{5} 4 d^{3} P_{1}$ & $1.07 \mathrm{E}+00$ & $2.91 \mathrm{E}+13$ & $1.51 \mathrm{E}-02$ & 5.33E-05 & $9.90 \mathrm{E}-01$ \\
\hline $2 s^{2} 2 p^{61} s_{0}$ & $2 \mathrm{~s}^{2} 2 \mathrm{p}^{5} 4 \mathrm{~d}^{1} \mathrm{P}_{1}$ & $1.07 E+00$ & $9.51 \mathrm{E}+14$ & $4.89 \mathrm{E}-01$ & $1.72 \mathrm{E}-03$ & $9.90 \mathrm{E}-01$ \\
\hline $2 s^{2} 2 p^{61} s_{0}$ & $2 s^{2} 2 p^{5} 4 s^{3} P_{1}$ & $9.85 \mathrm{E}-01$ & $1.84 \mathrm{E}+13$ & $8.05 \mathrm{E}-03$ & 2.61E-05 & $9.10 \mathrm{E}-01$ \\
\hline $2 s^{2} 2 p^{61} s_{0}$ & $2 \mathrm{~s}^{2} 2 \mathrm{p}^{5} 5 \mathrm{~s}^{1} \mathrm{P}_{1}$ & $9.75 \mathrm{E}-01$ & $3.08 \mathrm{E}+13$ & $1.32 \mathrm{E}-02$ & 4.23E-05 & $9.00 \mathrm{E}-01$ \\
\hline $2 s^{2} 2 p^{61} s_{0}$ & $2 s^{2} 2 p^{5} 5 d^{3} P_{1}$ & 9.65E-01 & $4.15 E+13$ & $1.74 \mathrm{E}-02$ & 5.51E-05 & $9.90 \mathrm{E}-01$ \\
\hline $2 s^{2} 2 p^{61} s_{0}$ & $2 s^{2} 2 p^{5} 5 d^{1} P_{1}$ & $9.64 \mathrm{E}-01$ & $2.22 \mathrm{E}+14$ & $9.27 \mathrm{E}-02$ & 2.94E-04 & $9.90 \mathrm{E}-01$ \\
\hline $2 s^{2} 2 p^{61} s_{0}$ & $2 s^{2} 2 p^{5} 4 d^{3} D_{1}$ & $9.63 \mathrm{E}-01$ & $8.08 \mathrm{E}+14$ & 3.37E-01 & $1.07 \mathrm{E}-03$ & $9.90 \mathrm{E}-01$ \\
\hline $2 s^{2} 2 p^{61} s_{0}$ & $2 s 2 p^{6} 4 p^{3} P_{1}$ & $9.45 \mathrm{E}-01$ & $1.34 \mathrm{E}+14$ & $5.40 \mathrm{E}-02$ & $1.68 \mathrm{E}-04$ & $9.80 \mathrm{E}-01$ \\
\hline $2 s^{2} 2 p^{61} s_{0}$ & $2 s 2 p^{6} 4 p^{1} P_{1}$ & 9.34E-01 & $2.25 \mathrm{E}+14$ & $8.82 \mathrm{E}-02$ & $2.71 \mathrm{E}-04$ & $9.80 \mathrm{E}-01$ \\
\hline $2 s^{2} 2 p^{61} s_{0}$ & $2 \mathrm{~s}^{2} 2 \mathrm{p}^{5} 6 \mathrm{~s}^{1} \mathrm{P}_{1}$ & $9.20 \mathrm{E}-01$ & $1.40 \mathrm{E}+13$ & $5.33 \mathrm{E}-03$ & $1.61 \mathrm{E}-05$ & 8.30E-01 \\
\hline $2 s^{2} 2 p^{61} s_{0}$ & $2 s^{2} 2 p^{5} 6 d^{3} P_{1}$ & $9.15 \mathrm{E}-01$ & $8.54 \mathrm{E}+12$ & $3.21 \mathrm{E}-03$ & 9.67E-06 & $9.70 \mathrm{E}-01$ \\
\hline $2 s^{2} 2 p^{61} s_{0}$ & $2 s^{2} 2 p^{5} 6 d^{1} P_{1}$ & $9.14 \mathrm{E}-01$ & $2.49 E+14$ & 9.33E-02 & $2.81 \mathrm{E}-04$ & $9.70 \mathrm{E}-01$ \\
\hline $2 s^{2} 2 p^{61} s_{0}$ & $2 \mathrm{~s}^{2} 2 \mathrm{p}^{5} 7 \mathrm{~s}^{1} \mathrm{P}_{1}$ & $8.90 \mathrm{E}-01$ & $7.57 \mathrm{E}+12$ & $2.70 \mathrm{E}-03$ & 7.90E-06 & $1.00 \mathrm{E}+00$ \\
\hline $2 s^{2} 2 p^{61} s_{0}$ & $2 s^{2} 2 p^{5} 7 d^{3} P_{1}$ & $8.87 \mathrm{E}-01$ & $5.44 \mathrm{E}+12$ & $1.93 \mathrm{E}-03$ & $5.62 \mathrm{E}-06$ & $1.00 E+00$ \\
\hline $2 s^{2} 2 p^{61} s_{0}$ & $2 \mathrm{~s}^{2} 2 \mathrm{p}^{5} 7 \mathrm{~d}^{1} \mathrm{P}_{1}$ & $8.86 \mathrm{E}-01$ & $1.41 \mathrm{E}+14$ & $4.98 \mathrm{E}-02$ & $1.45 \mathrm{E}-04$ & $1.00 E+00$ \\
\hline $2 s^{2} 2 p^{61} s_{0}$ & $2 s^{2} 2 p^{5} 5 s^{3} P_{1}$ & $8.84 \mathrm{E}-01$ & $7.43 E+12$ & $2.61 \mathrm{E}-03$ & 7.61E-06 & $8.20 \mathrm{E}-01$ \\
\hline $2 s^{2} 2 p^{61} s_{0}$ & $2 s^{2} 2 p^{5} 5 d^{3} D_{1}$ & $8.76 \mathrm{E}-01$ & $3.00 \mathrm{E}+14$ & $1.04 \mathrm{E}-01$ & $2.99 \mathrm{E}-04$ & $9.80 \mathrm{E}-01$ \\
\hline $2 s^{2} 2 p^{61} s_{0}$ & $2 s 2 p^{6} 5 p^{3} P_{1}$ & $8.53 \mathrm{E}-01$ & $7.41 \mathrm{E}+13$ & 2.43E-02 & $6.82 \mathrm{E}-05$ & $9.80 \mathrm{E}-01$ \\
\hline $2 s^{2} 2 p^{61} s_{0}$ & $2 \mathrm{~s}^{2} \mathrm{p}^{6} 5 \mathrm{p}^{1} \mathrm{P}_{1}$ & $8.49 \mathrm{E}-01$ & $1.26 \mathrm{E}+14$ & 4.07E-02 & $1.14 \mathrm{E}-04$ & 9.60E-01 \\
\hline $2 s^{2} 2 p^{61} s_{0}$ & $2 s^{2} 2 p^{5} 6 s^{3} P_{1}$ & 8.39E-01 & $4.83 E+12$ & $1.53 \mathrm{E}-03$ & $4.22 \mathrm{E}-06$ & 7.50E-01 \\
\hline $2 s^{2} 2 p^{61} s_{0}$ & $2 s^{2} 2 p^{5} 6 d^{3} D_{1}$ & $8.34 \mathrm{E}-01$ & $1.58 \mathrm{E}+14$ & 4.94E-02 & $1.36 \mathrm{E}-04$ & 9.60E-01 \\
\hline $2 s^{2} 2 p^{61} s_{0}$ & $2 s^{2} 2 p^{5} 7 s^{3} P_{1}$ & $8.14 \mathrm{E}-01$ & $2.62 \mathrm{E}+12$ & 7.81E-04 & $2.09 \mathrm{E}-06$ & $9.30 \mathrm{E}-01$ \\
\hline $2 s^{2} 2 p^{61} s_{0}$ & $2 s^{2} 2 p^{5} 7 d^{3} D_{1}$ & $8.11 \mathrm{E}-01$ & $9.29 \mathrm{E}+13$ & $2.75 \mathrm{E}-02$ & $7.34 \mathrm{E}-05$ & $1.00 \mathrm{E}+00$ \\
\hline
\end{tabular}

Table 7: Transition wavelength (in Å), transition probability in $\mathrm{s}^{-1}$, oscillator strength (dimension less), line strength (length form) for E1 transition using MCDF for Ne-like W

\begin{tabular}{|c|c|c|c|c|c|c|}
\hline $\mathrm{i}$ & $\mathrm{j}$ & $\lambda$ (in $\AA$ ) & $A_{j i}\left(s^{-1}\right)$ & $f_{i j}$ & $\mathrm{~S}_{\mathrm{ij}}(\mathrm{au})$ & Vel./len. \\
\hline $2 s^{2} 2 p^{6}{ }^{1} S_{0}$ & $2 s^{2} 2 p^{5} 3 s^{1} P_{1}$ & $1.49 \mathrm{E}+00$ & $1.54 \mathrm{E}+14$ & $1.55 \mathrm{E}-01$ & $7.60 \mathrm{E}-04$ & $9.90 \mathrm{E}-01$ \\
\hline $2 s^{2} 2 p^{6}{ }^{1} S_{0}$ & $2 s^{2} 2 p^{5} 3 d^{3} \mathrm{P}_{1}$ & $1.38 \mathrm{E}+00$ & $8.14 E+13$ & $6.96 \mathrm{E}-02$ & 3.16E-04 & $1.00 \mathrm{E}+00$ \\
\hline $2 s^{2} 2 p^{6}{ }^{1} S_{0}$ & $2 s^{2} 2 p^{5} 3 d{ }^{1} P_{1}$ & $1.36 \mathrm{E}+00$ & $2.79 E+15$ & $2.31 E+00$ & $1.04 \mathrm{E}-02$ & $1.00 E+00$ \\
\hline $2 s^{2} 2 p^{6}{ }^{1} S_{0}$ & $2 s^{2} 2 p^{5} 3 s^{3} P_{1}$ & $1.28 \mathrm{E}+00$ & $3.90 \mathrm{E}+13$ & $2.88 \mathrm{E}-02$ & $1.21 \mathrm{E}-04$ & $9.70 \mathrm{E}-01$ \\
\hline $2 s^{2} 2 p^{6}{ }^{1} S_{0}$ & $2 s 2 p^{6} 3 p^{3} P_{1}$ & $1.20 \mathrm{E}+00$ & $7.99 \mathrm{E}+14$ & $5.18 \mathrm{E}-01$ & $2.05 \mathrm{E}-03$ & $1.00 E+00$ \\
\hline $2 s^{2} 2 p^{6}{ }^{1} S_{0}$ & $2 s^{2} 2 p^{5} 3 d^{3} D_{1}$ & $1.19 \mathrm{E}+00$ & $1.33 E+15$ & 8.46E-01 & 3.32E-03 & $1.00 \mathrm{E}+00$ \\
\hline $2 s^{2} 2 p^{6}{ }^{1} S_{0}$ & $2 s 2 p^{6} 3 p{ }^{1} P_{1}$ & $1.16 \mathrm{E}+00$ & $4.46 \mathrm{E}+14$ & $2.68 \mathrm{E}-01$ & $1.02 \mathrm{E}-03$ & $1.00 \mathrm{E}+00$ \\
\hline $2 s^{2} 2 p^{6}{ }^{1} S_{0}$ & $2 s^{2} 2 p^{5} 4 s^{1} P_{1}$ & $1.07 \mathrm{E}+00$ & $5.44 E+13$ & $2.79 \mathrm{E}-02$ & $9.78 \mathrm{E}-05$ & $9.50 \mathrm{E}-01$ \\
\hline $2 s^{2} 2 p^{6}{ }^{1} S_{0}$ & $2 s^{2} 2 p^{5} 4 d^{3} P_{1}$ & $1.04 \mathrm{E}+00$ & $3.24 E+13$ & $1.58 \mathrm{E}-02$ & $5.43 \mathrm{E}-05$ & $9.90 \mathrm{E}-01$ \\
\hline $2 s^{2} 2 p^{6}{ }^{1} S_{0}$ & $2 s^{2} 2 p^{5} 4 d^{1} P_{1}$ & $1.04 \mathrm{E}+00$ & $1.01 \mathrm{E}+15$ & 4.87E-01 & $1.66 \mathrm{E}-03$ & $9.90 \mathrm{E}-01$ \\
\hline $2 s^{2} 2 p^{6}{ }^{1} S_{0}$ & $2 s^{2} 2 p^{5} 4 s^{3} P_{1}$ & $9.53 \mathrm{E}-01$ & $1.96 \mathrm{E}+13$ & $8.01 \mathrm{E}-03$ & $2.51 \mathrm{E}-05$ & $9.10 \mathrm{E}-01$ \\
\hline $2 s^{2} 2 p^{6}{ }^{1} S_{0}$ & $2 s^{2} 2 p^{5} 5 s^{1} P_{1}$ & $9.46 \mathrm{E}-01$ & $3.21 \mathrm{E}+13$ & $1.29 \mathrm{E}-02$ & 4.03E-05 & $9.10 \mathrm{E}-01$ \\
\hline $2 s^{2} 2 p^{6}{ }^{1} S_{0}$ & $2 s^{2} 2 p^{5} 5 d^{3} P_{1}$ & $9.36 \mathrm{E}-01$ & $2.08 \mathrm{E}+13$ & $8.21 \mathrm{E}-03$ & 2.53E-05 & $9.90 \mathrm{E}-01$ \\
\hline $2 s^{2} 2 p^{6}{ }^{1} S_{0}$ & $2 s^{2} 2 p^{5} 5 d \quad{ }^{1} P_{1}$ & $9.34 \mathrm{E}-01$ & $2.29 E+14$ & 8.99E-02 & 2.77E-04 & $9.80 \mathrm{E}-01$ \\
\hline $2 s^{2} 2 p^{6}{ }^{1} S_{0}$ & $2 s^{2} 2 p^{5} 4 d^{3} D_{1}$ & $9.32 \mathrm{E}-01$ & $8.88 \mathrm{E}+14$ & 3.47E-01 & 1.07E-03 & $9.90 \mathrm{E}-01$ \\
\hline $2 s^{2} 2 p^{6} S_{0}$ & $2 s 2 p^{6} 4 p{ }^{3} P_{1}$ & $9.14 \mathrm{E}-01$ & $1.44 \mathrm{E}+14$ & $5.40 \mathrm{E}-02$ & $1.63 \mathrm{E}-04$ & $9.80 \mathrm{E}-01$ \\
\hline $2 s^{2} 2 p^{6}{ }^{1} S_{0}$ & $2 s 2 p^{6} 4 p{ }^{1} P_{1}$ & $9.04 \mathrm{E}-01$ & $2.38 E+14$ & 8.74E-02 & $2.60 \mathrm{E}-04$ & $9.80 \mathrm{E}-01$ \\
\hline $2 s^{2} 2 p^{6}{ }^{1} S_{0}$ & $2 s^{2} 2 p^{5} 6 s{ }^{1} P_{1}$ & $8.93 \mathrm{E}-01$ & $1.48 \mathrm{E}+13$ & $5.30 \mathrm{E}-03$ & $1.56 \mathrm{E}-05$ & 8.40E-01 \\
\hline $2 s^{2} 2 p^{6}{ }^{1} S_{0}$ & $2 s^{2} 2 p^{5} 6 d^{3} P_{1}$ & 8.87E-01 & $9.38 \mathrm{E}+12$ & $3.32 \mathrm{E}-03$ & $9.71 \mathrm{E}-06$ & $9.70 \mathrm{E}-01$ \\
\hline $2 s^{2} 2 p^{6}{ }^{1} S_{0}$ & $2 s^{2} 2 p^{5} 6 d^{1} P_{1}$ & 8.86E-01 & $2.63 E+14$ & $9.29 \mathrm{E}-02$ & $2.71 \mathrm{E}-04$ & $9.70 \mathrm{E}-01$ \\
\hline $2 s^{2} 2 p^{6}{ }^{1} S_{0}$ & $2 s^{2} 2 p^{5} 7 s^{1} P_{1}$ & 8.64E-01 & $8.04 E+12$ & $2.70 \mathrm{E}-03$ & 7.67E-06 & $1.00 E+00$ \\
\hline $2 s^{2} 2 p^{6}{ }^{1} S_{0}$ & $2 s^{2} 2 p^{5} 7 d^{3} P_{1}$ & $8.61 \mathrm{E}-01$ & $5.89 \mathrm{E}+12$ & $1.96 \mathrm{E}-03$ & $5.56 \mathrm{E}-06$ & $1.00 \mathrm{E}+00$ \\
\hline $2 s^{2} 2 p^{6}{ }^{1} S_{0}$ & $2 s^{2} 2 p^{5} 7 d^{1} P_{1}$ & $8.60 \mathrm{E}-01$ & $1.50 \mathrm{E}+14$ & 4.97E-02 & $1.41 \mathrm{E}-04$ & $1.00 \mathrm{E}+00$ \\
\hline $2 s^{2} 2 p^{6}{ }^{1} S_{0}$ & $2 s^{2} 2 p^{5} 5 s^{3} P_{1}$ & $8.56 \mathrm{E}-01$ & $8.84 E+12$ & $2.91 \mathrm{E}-03$ & 8.20E-06 & 8.30E-01 \\
\hline $2 s^{2} 2 p^{6}{ }^{1} S_{0}$ & $2 s^{2} 2 p^{5} 5 d^{3} D_{1}$ & $8.47 \mathrm{E}-01$ & $3.18 \mathrm{E}+14$ & $1.03 \mathrm{E}-01$ & $2.86 \mathrm{E}-04$ & $9.80 \mathrm{E}-01$ \\
\hline $2 s^{2} 2 p^{6}{ }^{1} S_{0}$ & $2 s 2 p^{6} 5 p^{3} P_{1}$ & $8.26 \mathrm{E}-01$ & $7.89 E+13$ & $2.42 \mathrm{E}-02$ & $6.59 \mathrm{E}-05$ & $9.80 \mathrm{E}-01$ \\
\hline $2 s^{2} 2 p^{6}{ }^{1} S_{0}$ & $2 s 2 p^{6} 5 p^{1} P_{1}$ & $8.22 \mathrm{E}-01$ & $1.33 E+14$ & 4.03E-02 & 1.09E-04 & $9.60 \mathrm{E}-01$ \\
\hline $2 s^{2} 2 p^{6}{ }^{1} S_{0}$ & $2 s^{2} 2 p^{5} 6 s^{3} P_{1}$ & 8.11E-01 & $5.13 E+12$ & $1.52 \mathrm{E}-03$ & 4.06E-06 & 7.60E-01 \\
\hline $2 s^{2} 2 p^{6}{ }^{1} S_{0}$ & $2 s^{2} 2 p^{5} 6 d^{3} D_{1}$ & 8.07E-01 & $1.68 \mathrm{E}+14$ & 4.92E-02 & $1.31 \mathrm{E}-04$ & $9.60 \mathrm{E}-01$ \\
\hline $2 s^{2} 2 p^{6}{ }^{1} S_{0}$ & $2 s^{2} 2 p^{5} 7 s^{3} P_{1}$ & 7.87E-01 & $2.79 E+12$ & $7.78 \mathrm{E}-04$ & $2.02 \mathrm{E}-06$ & $9.30 \mathrm{E}-01$ \\
\hline $2 s^{2} 2 p^{6}{ }^{1} S_{0}$ & $2 s^{2} 2 p^{5} 7 d^{3} D_{1}$ & $7.85 \mathrm{E}-01$ & $9.90 E+13$ & $2.74 \mathrm{E}-02$ & $7.08 \mathrm{E}-05$ & $1.00 \mathrm{E}+00$ \\
\hline
\end{tabular}

Table 8: Transition wavelength (in Å), transition probability in $\mathrm{s}^{-1}$, oscillator strength (dimensionless), line strength (length form) for E1 transition using MCDF for Ne-like Re 


\begin{tabular}{|c|c|c|c|c|c|c|}
\hline i & j & $\lambda$ (in $\AA$ ) & $A_{j i}\left(s^{-1}\right)$ & $f_{i j}$ & $S_{\mathrm{ij}}(\mathrm{au})$ & Vel./len. \\
\hline $2 s^{2} 2 p^{61} S_{0}$ & $2 s^{2} 2 p^{5} 3 s^{11} P_{1}$ & $1.45 \mathrm{E}+00$ & $1.65 \mathrm{E}+14$ & $1.56 \mathrm{E}-01$ & 7.47E-04 & $9.90 \mathrm{E}-01$ \\
\hline $2 s^{2} 2 p^{61} S_{0}$ & $2 s^{2} 2 p^{5} 3 d^{13} P_{1}$ & $1.34 \mathrm{E}+00$ & $9.14 E+13$ & 7.37E-02 & $3.25 \mathrm{E}-04$ & $1.00 E+00$ \\
\hline $2 s^{2} 2 p^{61} s_{0}$ & $2 s^{2} 2 p^{5} 3 d^{11} P_{1}$ & $1.32 \mathrm{E}+00$ & $2.95 \mathrm{E}+15$ & $2.31 \mathrm{E}+00$ & $1.00 \mathrm{E}-02$ & $1.00 \mathrm{E}+00$ \\
\hline $2 s^{2} 2 p^{61} S_{0}$ & $2 s^{2} 2 p^{5} 3 s^{13} P_{1}$ & $1.24 \mathrm{E}+00$ & $4.21 E+13$ & $2.91 \mathrm{E}-02$ & $1.19 \mathrm{E}-04$ & $9.70 \mathrm{E}-01$ \\
\hline $2 s^{2} 2 p^{61} S_{0}$ & $2 s^{1} 2 p^{6} 3 p^{13} P_{1}$ & $1.16 \mathrm{E}+00$ & $7.61 E+14$ & $4.62 \mathrm{E}-01$ & $1.77 \mathrm{E}-03$ & $1.00 \mathrm{E}+00$ \\
\hline $2 s^{2} 2 p^{61} S_{0}$ & $2 s^{2} 2 p^{5} 3 d^{13} D_{1}$ & $1.15 \mathrm{E}+00$ & $1.50 \mathrm{E}+15$ & $8.94 \mathrm{E}-01$ & $3.39 \mathrm{E}-03$ & $1.00 \mathrm{E}+00$ \\
\hline $2 s^{2} 2 p^{61} S_{0}$ & $2 s^{1} 2 p^{6} 3 p^{11} P_{1}$ & $1.12 \mathrm{E}+00$ & $4.70 \mathrm{E}+14$ & $2.65 \mathrm{E}-01$ & $9.76 \mathrm{E}-04$ & $1.00 \mathrm{E}+00$ \\
\hline $2 s^{2} 2 p^{61} S_{0}$ & $2 s^{2} 2 p^{5} 4 s^{11} P_{1}$ & $1.04 \mathrm{E}+00$ & $5.81 E+13$ & $2.80 \mathrm{E}-02$ & $9.56 \mathrm{E}-05$ & $9.50 \mathrm{E}-01$ \\
\hline $2 s^{2} 2 p^{61} s_{0}$ & $2 s^{2} 2 p^{5} 4 d^{13} P_{1}$ & $1.01 \mathrm{E}+00$ & $3.60 E+13$ & $1.65 \mathrm{E}-02$ & 5.51E-05 & $9.90 \mathrm{E}-01$ \\
\hline $2 s^{2} 2 p^{61} s_{0}$ & $2 s^{2} 2 p^{5} 4 d^{11} P_{1}$ & $1.01 E+00$ & $1.07 \mathrm{E}+15$ & 4.86E-01 & $1.61 \mathrm{E}-03$ & $9.90 \mathrm{E}-01$ \\
\hline $2 s^{2} 2 p^{61} S_{0}$ & $2 s^{2} 2 p^{5} 4 s^{13} p_{1}$ & $9.22 \mathrm{E}-01$ & $2.06 \mathrm{E}+13$ & $7.87 \mathrm{E}-03$ & $2.39 \mathrm{E}-05$ & $9.10 \mathrm{E}-01$ \\
\hline $2 s^{2} 2 p^{61} S_{0}$ & $2 s^{2} 2 p^{5} 5 s^{11} P_{1}$ & $9.19 \mathrm{E}-01$ & $3.41 \mathrm{E}+13$ & $1.29 \mathrm{E}-02$ & $3.91 \mathrm{E}-05$ & $9.10 \mathrm{E}-01$ \\
\hline $2 s^{2} 2 p^{61} s_{0}$ & $2 s^{2} 2 p^{5} 5 d^{13} P_{1}$ & $9.09 \mathrm{E}-01$ & $2.09 \mathrm{E}+13$ & 7.77E-03 & $2.32 \mathrm{E}-05$ & $9.90 \mathrm{E}-01$ \\
\hline $2 s^{2} 2 p^{61} S_{0}$ & $2 s^{2} 2 p^{5} 5 d^{11} P_{1}$ & $9.07 \mathrm{E}-01$ & $3.94 \mathrm{E}+14$ & $1.46 \mathrm{E}-01$ & $4.35 \mathrm{E}-04$ & $9.80 \mathrm{E}-01$ \\
\hline $2 s^{2} 2 p^{61} S_{0}$ & $2 s^{2} 2 p^{5} 4 d^{13} D_{1}$ & $9.02 E-01$ & $7.93 E+14$ & $2.90 \mathrm{E}-01$ & $8.62 \mathrm{E}-04$ & $9.90 \mathrm{E}-01$ \\
\hline $2 s^{2} 2 p^{61} S_{0}$ & $2 s^{1} 2 p^{6} 4 p^{13} P_{1}$ & 8.85E-01 & $1.53 \mathrm{E}+14$ & $5.40 \mathrm{E}-02$ & $1.57 \mathrm{E}-04$ & $9.80 \mathrm{E}-01$ \\
\hline $2 s^{2} 2 p^{61} S_{0}$ & $2 s^{1} 2 p^{6} 4 p^{11} P_{1}$ & $8.75 \mathrm{E}-01$ & $2.52 E+14$ & $8.66 \mathrm{E}-02$ & $2.49 \mathrm{E}-04$ & $9.80 \mathrm{E}-01$ \\
\hline $2 s^{2} 2 p^{61} S_{0}$ & $2 s^{2} 2 p^{5} 6 s^{11} P_{1}$ & 8.66E-01 & $1.56 E+13$ & $5.26 \mathrm{E}-03$ & $1.50 \mathrm{E}-05$ & $8.40 \mathrm{E}-01$ \\
\hline $2 s^{2} 2 p^{61} s_{0}$ & $2 s^{2} 2 p^{5} 6 d^{13} P_{1}$ & $8.61 \mathrm{E}-01$ & $1.03 \mathrm{E}+13$ & $3.42 \mathrm{E}-03$ & $9.71 \mathrm{E}-06$ & $9.70 \mathrm{E}-01$ \\
\hline $2 s^{2} 2 p^{61} S_{0}$ & $2 s^{2} 2 p^{5} 6 d^{11} P_{1}$ & $8.60 \mathrm{E}-01$ & $2.78 \mathrm{E}+14$ & $9.26 \mathrm{E}-02$ & $2.62 \mathrm{E}-04$ & $9.70 \mathrm{E}-01$ \\
\hline $2 s^{2} 2 p^{61} S_{0}$ & $2 s^{2} 2 p^{5} 7 s^{11} P_{1}$ & 8.38E-01 & $8.54 \mathrm{E}+12$ & $2.70 \mathrm{E}-03$ & $7.45 \mathrm{E}-06$ & $1.00 E+00$ \\
\hline $2 s^{2} 2 p^{61} s_{0}$ & $2 s^{2} 2 p^{5} 7 d^{13} P_{1}$ & $8.35 \mathrm{E}-01$ & $6.42 \mathrm{E}+12$ & $2.01 \mathrm{E}-03$ & $5.54 \mathrm{E}-06$ & $1.00 \mathrm{E}+00$ \\
\hline $2 s^{2} 2 p^{61} s_{0}$ & $2 s^{2} 2 p^{5} 7 d^{11} P_{1}$ & $8.35 \mathrm{E}-01$ & $1.59 E+14$ & $4.98 \mathrm{E}-02$ & $1.37 \mathrm{E}-04$ & $1.00 \mathrm{E}+00$ \\
\hline $2 s^{2} 2 p^{61} S_{0}$ & $2 s^{2} 2 p^{5} 5 s^{13} P_{1}$ & $8.28 \mathrm{E}-01$ & $9.68 \mathrm{E}+12$ & $2.98 \mathrm{E}-03$ & 8.14E-06 & 8.30E-01 \\
\hline $2 s^{2} 2 p^{61} S_{0}$ & $2 s^{2} 2 p^{5} 5 d^{13} D_{1}$ & $8.20 \mathrm{E}-01$ & $3.37 E+14$ & $1.02 \mathrm{E}-01$ & $2.75 \mathrm{E}-04$ & $9.80 \mathrm{E}-01$ \\
\hline $2 s^{2} 2 p^{61} S_{0}$ & $2 s^{1} 2 p^{6} 5 p^{13} P_{1}$ & $8.00 \mathrm{E}-01$ & $8.41 E+13$ & $2.42 \mathrm{E}-02$ & $6.37 \mathrm{E}-05$ & $9.80 \mathrm{E}-01$ \\
\hline $2 s^{2} 2 p^{61} S_{0}$ & $2 s^{1} 2 p^{6} 5 p^{11} P_{1}$ & 7.95E-01 & $1.40 \mathrm{E}+14$ & $3.98 \mathrm{E}-02$ & $1.04 \mathrm{E}-04$ & $9.60 \mathrm{E}-01$ \\
\hline $2 s^{2} 2 p^{61} S_{0}$ & $2 s^{2} 2 p^{5} 6 s^{13} P_{1}$ & $7.85 \mathrm{E}-01$ & $5.44 \mathrm{E}+12$ & $1.51 \mathrm{E}-03$ & $3.90 \mathrm{E}-06$ & $7.60 \mathrm{E}-01$ \\
\hline $2 s^{2} 2 p^{61} s_{0}$ & $2 s^{2} 2 p^{5} 6 d^{13} D_{1}$ & $7.81 \mathrm{E}-01$ & $1.78 \mathrm{E}+14$ & $4.89 \mathrm{E}-02$ & $1.26 \mathrm{E}-04$ & $9.70 \mathrm{E}-01$ \\
\hline $2 s^{2} 2 p^{61} S_{0}$ & $2 s^{2} 2 p^{5} 7 s^{13} P_{1}$ & 7.62E-01 & $2.97 E+12$ & $7.75 \mathrm{E}-04$ & $1.94 \mathrm{E}-06$ & $9.30 \mathrm{E}-01$ \\
\hline $2 s^{2} 2 p^{61} s_{0}$ & $2 s^{2} 2 p^{5} 7 d^{13} D_{1}$ & $7.60 \mathrm{E}-01$ & $1.05 E+14$ & $2.74 \mathrm{E}-02$ & $6.84 \mathrm{E}-05$ & $1.00 \mathrm{E}+00$ \\
\hline
\end{tabular}

Table 9: Transition wavelength (in Å), transition probability in $\mathrm{s}^{-1}$, oscillator strength (dimensionless), line strength (length form) for E2, M1 and $\mathrm{M} 2$ transition using MCDF for Ne-like W

\begin{tabular}{|c|c|c|c|c|c|c|}
\hline $\mathrm{i}$ & j & $\lambda$ (in $\AA$ ) & $A_{j i}\left(s^{-1}\right)$ & $f_{i j}$ & $S_{\mathrm{ijj}}(\mathrm{au})$ & Type \\
\hline 1 & 2 & $1.49 \mathrm{E}+00$ & $4.32 \mathrm{E}+09$ & $7.24 \mathrm{E}-06$ & $1.08 \mathrm{E}-02$ & M2 \\
\hline 1 & 4 & $1.47 \mathrm{E}+00$ & $3.07 E+10$ & 2.97E-05 & $1.08 \mathrm{E}-02$ & M1 \\
\hline 1 & 5 & $1.47 E+00$ & $9.28 \mathrm{E}+11$ & $1.50 \mathrm{E}-03$ & $2.82 \mathrm{E}-05$ & E2 \\
\hline 1 & 6 & $1.41 E+00$ & $1.07 \mathrm{E}+10$ & $9.46 \mathrm{E}-06$ & $3.29 \mathrm{E}-03$ & M1 \\
\hline 1 & 8 & $1.40 E+00$ & $9.18 \mathrm{E}+11$ & $1.35 \mathrm{E}-03$ & $2.23 \mathrm{E}-05$ & E2 \\
\hline 1 & 13 & $1.38 \mathrm{E}+00$ & $2.28 \mathrm{E}+08$ & $3.24 \mathrm{E}-07$ & $3.79 \mathrm{E}-04$ & M2 \\
\hline 1 & 15 & $1.36 \mathrm{E}+00$ & $1.21 \mathrm{E}+11$ & $1.69 \mathrm{E}-04$ & $1.92 \mathrm{E}-01$ & M2 \\
\hline 1 & 20 & $1.26 \mathrm{E}+00$ & $1.30 \mathrm{E}+09$ & $9.30 \mathrm{E}-07$ & $2.90 \mathrm{E}-04$ & M1 \\
\hline 1 & 22 & $1.22 \mathrm{E}+00$ & $2.25 E+08$ & $1.51 \mathrm{E}-07$ & $4.55 \mathrm{E}-05$ & M1 \\
\hline 1 & 24 & $1.21 \mathrm{E}+00$ & $1.11 \mathrm{E}+12$ & $1.23 \mathrm{E}-03$ & $1.31 \mathrm{E}-05$ & E2 \\
\hline 1 & 25 & $1.21 E+00$ & $2.80 \mathrm{E}+10$ & $1.85 \mathrm{E}-05$ & $5.55 \mathrm{E}-03$ & M1 \\
\hline 1 & 28 & $1.19 \mathrm{E}+00$ & $2.96 \mathrm{E}+09$ & 3.17E-06 & $2.42 \mathrm{E}-03$ & M2 \\
\hline 1 & 30 & $1.18 \mathrm{E}+00$ & $3.42 \mathrm{E}+10$ & $3.60 \mathrm{E}-05$ & $2.67 \mathrm{E}-02$ & M2 \\
\hline 1 & 32 & $1.16 \mathrm{E}+00$ & $2.14 \mathrm{E}+10$ & $2.15 \mathrm{E}-05$ & $1.49 \mathrm{E}-02$ & M2 \\
\hline 1 & 34 & $1.14 \mathrm{E}+00$ & $5.64 \mathrm{E}+08$ & $3.30 \mathrm{E}-07$ & $9.30 \mathrm{E}-05$ & M1 \\
\hline 1 & 35 & $1.14 \mathrm{E}+00$ & $4.96 \mathrm{E}+12$ & $4.83 \mathrm{E}-03$ & $4.25 \mathrm{E}-05$ & E2 \\
\hline 1 & 37 & $1.13 E+00$ & $1.20 \mathrm{E}+13$ & $1.15 \mathrm{E}-02$ & $9.86 \mathrm{E}-05$ & E2 \\
\hline 1 & 38 & $1.07 E+00$ & $2.98 \mathrm{E}+09$ & $2.55 \mathrm{E}-06$ & $1.38 \mathrm{E}-03$ & M2 \\
\hline 1 & 40 & $1.06 \mathrm{E}+00$ & $1.73 \mathrm{E}+10$ & $8.74 \mathrm{E}-06$ & $2.29 \mathrm{E}-03$ & M1 \\
\hline 1 & 41 & $1.06 \mathrm{E}+00$ & $3.19 \mathrm{E}+11$ & $2.70 \mathrm{E}-04$ & $1.92 \mathrm{E}-06$ & E2 \\
\hline 1 & 43 & $1.05 \mathrm{E}+00$ & $8.16 \mathrm{E}+09$ & $4.03 \mathrm{E}-06$ & $1.04 \mathrm{E}-03$ & M1 \\
\hline 1 & 44 & $1.05 E+00$ & $3.73 \mathrm{E}+11$ & $3.06 \mathrm{E}-04$ & $2.09 \mathrm{E}-06$ & E2 \\
\hline 1 & 49 & $1.04 \mathrm{E}+00$ & $7.19 \mathrm{E}+07$ & $5.85 \mathrm{E}-08$ & $2.95 \mathrm{E}-05$ & M2 \\
\hline 1 & 51 & $1.04 E+00$ & $6.92 E+10$ & $5.59 \mathrm{E}-05$ & $2.80 \mathrm{E}-02$ & M2 \\
\hline 1 & 54 & $1.04 E+00$ & $1.38 \mathrm{E}+08$ & $6.67 \mathrm{E}-08$ & $1.71 \mathrm{E}-05$ & M1 \\
\hline 1 & 56 & $1.04 \mathrm{E}+00$ & $7.93 \mathrm{E}+11$ & $6.37 \mathrm{E}-04$ & $4.21 \mathrm{E}-06$ & E2 \\
\hline 1 & 59 & $1.03 E+00$ & $7.60 E+12$ & $6.09 \mathrm{E}-03$ & $4.01 \mathrm{E}-05$ & E2 \\
\hline 1 & 64 & $9.49 \mathrm{E}-01$ & $9.64 \mathrm{E}+08$ & $3.90 \mathrm{E}-07$ & $9.16 \mathrm{E}-05$ & M1 \\
\hline 1 & 66 & $9.46 \mathrm{E}-01$ & $2.10 \mathrm{E}+09$ & $1.41 \mathrm{E}-06$ & $5.35 \mathrm{E}-04$ & M2 \\
\hline 1 & 68 & $9.44 \mathrm{E}-01$ & $9.17 E+09$ & $3.67 \mathrm{E}-06$ & $8.58 \mathrm{E}-04$ & M1 \\
\hline 1 & 69 & $9.44 \mathrm{E}-01$ & $1.87 \mathrm{E}+11$ & $1.25 \mathrm{E}-04$ & $6.27 \mathrm{E}-07$ & E2 \\
\hline 1 & 70 & $9.38 \mathrm{E}-01$ & $2.62 E+09$ & 1.04E-06 & $2.40 \mathrm{E}-04$ & M1 \\
\hline
\end{tabular}




\begin{tabular}{|c|c|c|c|c|c|c|}
\hline 1 & 72 & $9.38 \mathrm{E}-01$ & $2.46 \mathrm{E}+11$ & $1.62 \mathrm{E}-04$ & $7.98 \mathrm{E}-07$ & E2 \\
\hline 1 & 74 & 9.37E-01 & $1.39 \mathrm{E}+10$ & 5.49E-06 & $1.27 \mathrm{E}-03$ & M1 \\
\hline 1 & 75 & 9.37E-01 & $5.39 \mathrm{E}+11$ & 3.55E-04 & $1.74 \mathrm{E}-06$ & E2 \\
\hline 1 & 79 & $9.36 \mathrm{E}-01$ & $9.21 E+07$ & $6.05 \mathrm{E}-08$ & $2.22 \mathrm{E}-05$ & M2 \\
\hline 1 & 81 & $9.35 \mathrm{E}-01$ & $4.13 \mathrm{E}+10$ & $2.71 \mathrm{E}-05$ & $9.89 \mathrm{E}-03$ & M2 \\
\hline 1 & 84 & $9.34 \mathrm{E}-01$ & $1.24 \mathrm{E}+08$ & 4.87E-08 & $1.12 \mathrm{E}-05$ & M1 \\
\hline 1 & 86 & $9.34 \mathrm{E}-01$ & $5.65 \mathrm{E}+11$ & 3.69E-04 & $1.79 \mathrm{E}-06$ & E2 \\
\hline 1 & 89 & 9.33E-01 & $5.28 \mathrm{E}+12$ & 3.45E-03 & 1.67E-05 & E2 \\
\hline 1 & 92 & $9.33 \mathrm{E}-01$ & $3.75 E+07$ & $2.44 \mathrm{E}-08$ & 8.87E-06 & M2 \\
\hline 1 & 100 & $9.33 \mathrm{E}-01$ & $2.42 \mathrm{E}+09$ & $1.58 \mathrm{E}-06$ & $5.74 \mathrm{E}-04$ & M2 \\
\hline 1 & 102 & $9.30 \mathrm{E}-01$ & $1.74 \mathrm{E}+10$ & 1.13E-05 & 4.05E-03 & M2 \\
\hline 1 & 105 & $9.28 \mathrm{E}-01$ & $4.32 \mathrm{E}+12$ & $2.78 \mathrm{E}-03$ & $1.32 \mathrm{E}-05$ & E2 \\
\hline 1 & 108 & $9.19 \mathrm{E}-01$ & 4.17E+09 & $1.58 \mathrm{E}-06$ & 3.59E-04 & M1 \\
\hline 1 & 112 & $9.04 \mathrm{E}-01$ & $1.88 \mathrm{E}+10$ & 1.15E-05 & $3.80 \mathrm{E}-03$ & $\mathrm{M} 2$ \\
\hline 1 & 114 & $9.00 \mathrm{E}-01$ & $3.16 \mathrm{E}+08$ & $1.15 \mathrm{E}-07$ & $2.56 \mathrm{E}-05$ & M1 \\
\hline 1 & 115 & $9.00 \mathrm{E}-01$ & $1.16 \mathrm{E}+12$ & 7.03E-04 & 3.05E-06 & E2 \\
\hline 1 & 117 & 8.97E-01 & $2.71 E+12$ & $1.63 \mathrm{E}-03$ & $7.02 \mathrm{E}-06$ & E2 \\
\hline 1 & 118 & 8.95E-01 & $1.15 \mathrm{E}+06$ & $6.91 \mathrm{E}-10$ & $2.22 \mathrm{E}-07$ & M2 \\
\hline 1 & 122 & 8.93E-01 & $1.09 \mathrm{E}+09$ & $6.48 \mathrm{E}-07$ & $2.06 \mathrm{E}-04$ & M2 \\
\hline 1 & 124 & 8.91E-01 & $5.31 E+09$ & 1.90E-06 & $4.18 \mathrm{E}-04$ & M1 \\
\hline 1 & 125 & 8.91E-01 & $1.29 \mathrm{E}+11$ & $7.69 \mathrm{E}-05$ & $3.24 \mathrm{E}-07$ & E2 \\
\hline 1 & 126 & 8.89E-01 & $2.41 E+09$ & $8.56 \mathrm{E}-07$ & $1.88 \mathrm{E}-04$ & M1 \\
\hline 1 & 128 & $8.88 \mathrm{E}-01$ & $1.70 \mathrm{E}+11$ & $1.01 \mathrm{E}-04$ & $4.20 \mathrm{E}-07$ & E2 \\
\hline 1 & 133 & 8.87E-01 & $1.60 \mathrm{E}+07$ & 9.43E-09 & 2.95E-06 & M2 \\
\hline 1 & 135 & $8.87 \mathrm{E}-01$ & $2.37 \mathrm{E}+10$ & $1.39 \mathrm{E}-05$ & 4.35E-03 & M2 \\
\hline 1 & 138 & 8.64E-01 & $6.98 \mathrm{E}+08$ & 3.90E-07 & 1.12E-04 & M2 \\
\hline 1 & 140 & $8.63 \mathrm{E}-01$ & $3.30 \mathrm{E}+09$ & 1.10E-06 & $2.36 \mathrm{E}-04$ & M1 \\
\hline 1 & 141 & $8.63 \mathrm{E}-01$ & $4.92 \mathrm{E}+10$ & $2.75 \mathrm{E}-05$ & $1.05 \mathrm{E}-07$ & E2 \\
\hline 1 & 143 & $8.61 \mathrm{E}-01$ & $1.20 \mathrm{E}+09$ & 3.99E-07 & $8.50 \mathrm{E}-05$ & M1 \\
\hline 1 & 144 & $8.61 \mathrm{E}-01$ & $8.01 \mathrm{E}+10$ & 4.45E-05 & $1.69 \mathrm{E}-07$ & E2 \\
\hline 1 & 149 & 8.61E-01 & $1.09 \mathrm{E}+07$ & 6.07E-09 & $1.73 \mathrm{E}-06$ & M2 \\
\hline 1 & 151 & $8.60 \mathrm{E}-01$ & $1.45 \mathrm{E}+10$ & 8.07E-06 & $2.30 \mathrm{E}-03$ & M2 \\
\hline 1 & 156 & 8.54E-01 & $6.83 E+08$ & $2.24 \mathrm{E}-07$ & 4.73E-05 & M1 \\
\hline 1 & 158 & $8.49 \mathrm{E}-01$ & $6.31 E+09$ & $2.05 \mathrm{E}-06$ & 4.30E-04 & M1 \\
\hline 1 & 159 & 8.49E-01 & $3.07 E+11$ & $1.66 \mathrm{E}-04$ & $6.05 \mathrm{E}-07$ & E2 \\
\hline 1 & 160 & 8.47E-01 & $1.05 \mathrm{E}+09$ & $5.65 \mathrm{E}-07$ & $1.54 \mathrm{E}-04$ & M2 \\
\hline 1 & 162 & 8.46E-01 & $1.11 \mathrm{E}+10$ & 5.93E-06 & $1.61 \mathrm{E}-03$ & M2 \\
\hline 1 & 165 & 8.45E-01 & $3.03 E+12$ & $1.62 \mathrm{E}-03$ & $5.84 \mathrm{E}-06$ & E2 \\
\hline 1 & 172 & $8.28 \mathrm{E}-01$ & $2.08 \mathrm{E}+09$ & $6.40 \mathrm{E}-07$ & $1.31 \mathrm{E}-04$ & M1 \\
\hline 1 & 176 & $8.22 \mathrm{E}-01$ & $1.25 \mathrm{E}+10$ & $6.32 \mathrm{E}-06$ & 1.57E-03 & M2 \\
\hline 1 & 178 & $8.20 \mathrm{E}-01$ & $1.66 \mathrm{E}+08$ & $5.03 \mathrm{E}-08$ & $1.02 \mathrm{E}-05$ & M1 \\
\hline 1 & 179 & $8.20 \mathrm{E}-01$ & $4.97 E+11$ & $2.51 \mathrm{E}-04$ & $8.22 \mathrm{E}-07$ & E2 \\
\hline 1 & 181 & 8.19E-01 & $1.26 \mathrm{E}+12$ & $6.32 \mathrm{E}-04$ & 2.07E-06 & E2 \\
\hline 1 & 182 & $8.18 \mathrm{E}-01$ & $2.40 E+06$ & $1.20 \mathrm{E}-09$ & $2.95 \mathrm{E}-07$ & M2 \\
\hline 1 & 192 & $8.11 \mathrm{E}-01$ & $4.44 \mathrm{E}+08$ & $1.31 \mathrm{E}-07$ & $2.63 \mathrm{E}-05$ & M1 \\
\hline 1 & 194 & $8.08 \mathrm{E}-01$ & $3.91 E+09$ & 1.15E-06 & 2.30E-04 & $\mathrm{M} 1$ \\
\hline 1 & 195 & $8.08 \mathrm{E}-01$ & $2.44 \mathrm{E}+11$ & 1.19E-04 & 3.76E-07 & E2 \\
\hline 1 & 196 & 8.07E-01 & $6.61 E+08$ & 3.23E-07 & 7.60E-05 & M2 \\
\hline 1 & 198 & 8.07E-01 & $6.55 \mathrm{E}+09$ & $3.20 \mathrm{E}-06$ & 7.51E-04 & M2 \\
\hline 1 & 202 & 7.87E-01 & $2.14 \mathrm{E}+08$ & $5.95 \mathrm{E}-08$ & $1.16 \mathrm{E}-05$ & M1 \\
\hline 1 & 204 & 7.86E-01 & $2.54 \mathrm{E}+09$ & 7.04E-07 & 1.37E-04 & M1 \\
\hline 1 & 205 & 7.86E-01 & $1.09 \mathrm{E}+11$ & $5.06 \mathrm{E}-05$ & 1.46E-07 & E2 \\
\hline 1 & 206 & $7.85 \mathrm{E}-01$ & $4.14 \mathrm{E}+08$ & $1.91 \mathrm{E}-07$ & 4.13E-05 & M2 \\
\hline 1 & 208 & $7.85 \mathrm{E}-01$ & $4.09 \mathrm{E}+09$ & $1.89 \mathrm{E}-06$ & $4.08 \mathrm{E}-04$ & M2 \\
\hline
\end{tabular}

Table 10- comparison table of transition probability and oscillator strength for Z=74

\begin{tabular}{|c|c|c|c|c|c|c|c|c|}
\hline \multirow[t]{2}{*}{1} & \multirow[t]{2}{*}{ J } & \multicolumn{3}{|c|}{ Transition probability } & \multicolumn{2}{|c|}{ Oscillator strength } & \multicolumn{2}{|c|}{ Vel/Length ratio } \\
\hline & & $\begin{array}{l}\text { MRMP } \\
\text { [Ref. 34] }\end{array}$ & $\begin{array}{l}\text { GRASP } \\
\text { [Ref. 24] }\end{array}$ & $\begin{array}{l}\text { GRASP } \\
\text { Our result }\end{array}$ & $\begin{array}{l}\text { GRASP } \\
\text { [Ref. 24] }\end{array}$ & $\begin{array}{l}\text { GRASP } \\
\text { Our result }\end{array}$ & $\begin{array}{l}\text { GRASP } \\
\text { [Ref. 24] }\end{array}$ & $\begin{array}{l}\text { GRASP } \\
\text { Our } \\
\text { result }\end{array}$ \\
\hline $2 s^{2} 2 p^{61} S_{0}$ & $2 s^{2} 2 p^{5} 3 s^{1} P_{1}$ & $1.206 \mathrm{E}+14$ & $1.5309 \mathrm{E}+14$ & $1.5424 \mathrm{E}+14$ & $1.5354 \mathrm{E}-01$ & $1.5468 \mathrm{E}-01$ & $1.0 \mathrm{E}-00$ & $9.9 \mathrm{E}-01$ \\
\hline $2 s^{2} 2 p^{61} S_{0}$ & $2 s^{2} 2 p^{5} 3 d^{3} P_{1}$ & $6.551 \mathrm{E}+13$ & $8.2270 E+13$ & $8.1400 E+13$ & $7.0330 \mathrm{E}-02$ & $6.9574 \mathrm{E}-02$ & $9.8 \mathrm{E}-01$ & $1.0 \mathrm{E}+00$ \\
\hline $2 s^{2} 2 p^{61} S_{0}$ & $2 s^{2} 2 p^{5} 3 d^{1} P_{1}$ & $2.613 E+15$ & $2.8077 E+15$ & $2.7869 \mathrm{E}+15$ & $2.3320 E+00$ & $2.3142 E+00$ & $9.8 \mathrm{E}-01$ & $1.0 \mathrm{E}+00$ \\
\hline $2 s^{2} 2 p^{61} S_{0}$ & $2 s^{2} 2 p^{5} 3 s^{3} P_{1}$ & $2.694 \mathrm{E}+13$ & $3.8180 E+13$ & $3.9031 E+13$ & $2.8152 \mathrm{E}-02$ & $2.8777 \mathrm{E}-02$ & $9.9 \mathrm{E}-01$ & $9.7 \mathrm{E}-01$ \\
\hline $2 s^{2} 2 p^{61} S_{0}$ & $2 s 2 p^{6} 3 p^{3} P_{1}$ & $6.243 E+14$ & $7.7623 E+14$ & $7.9945 E+14$ & $5.0372 \mathrm{E}-01$ & $5.1842 \mathrm{E}-01$ & $1.0 \mathrm{E}-00$ & $1.0 \mathrm{E}+00$ \\
\hline $2 s^{2} 2 p^{61} S_{0}$ & $2 s^{2} 2 p^{5} 3 d^{3} D_{1}$ & $1.227 \mathrm{E}+15$ & $1.3590 E+15$ & $1.3258 \mathrm{E}+15$ & $8.6763 \mathrm{E}-01$ & $8.4636 \mathrm{E}-01$ & $9.8 \mathrm{E}-01$ & $1.0 \mathrm{E}+00$ \\
\hline $2 s^{2} 2 p^{61} S_{0}$ & $2 s 2 p^{6} 3 p^{1} P_{1}$ & $3.350 E+14$ & $4.4021 \mathrm{E}-14$ & $4.4580 E+14$ & 2.6529E-01 & 2.6847E-01 & $1.0 \mathrm{E}-00$ & $1.0 \mathrm{E}+00$ \\
\hline
\end{tabular}




\begin{tabular}{|c|c|c|c|c|c|c|c|c|}
\hline $2 s^{2} 2 p^{61} S_{0}$ & $2 s^{2} 2 p^{5} 4 s^{1} P_{1}$ & $4.193 \mathrm{E}+13$ & $5.3006 E+13$ & $5.4491 \mathrm{E}+13$ & $2.7134 \mathrm{E}-02$ & $2.7851 \mathrm{E}-02$ & $9.6 \mathrm{E}-01$ & $9.5 \mathrm{E}-01$ \\
\hline $2 s^{2} 2 p^{61} S_{0}$ & $2 s^{2} 2 p^{5} 4 d^{1} P_{1}$ & $1.021 \mathrm{E}+15$ & $1.0126 \mathrm{E}+15$ & $1.0073 E+15$ & 4.8992E-01 & 4.8721E-01 & $9.7 \mathrm{E}-01$ & 9.9E-01 \\
\hline $2 s^{2} 2 p^{61} s_{0}$ & $2 s^{2} 2 p^{5} 5 d{ }^{1} P_{1}$ & $2.365 E+14$ & $2.3392 E+14$ & $2.2900 E+14$ & $9.1872 \mathrm{E}-02$ & 8.9924E-02 & 9.7E-01 & $9.8 \mathrm{E}-01$ \\
\hline $2 s^{2} 2 p^{61} S_{0}$ & $2 s^{2} 2 p^{5} 4 d{ }^{3} D_{1}$ & $8.690 \mathrm{E}+14$ & $8.9169 E+14$ & $8.8822 E+14$ & 3.4857E-01 & 3.4717E-01 & 9.7E-01 & 9.9E-01 \\
\hline $2 s^{2} 2 p^{61} S_{0}$ & $2 s 2 p^{6} 4 p{ }^{3} P_{1}$ & $1.118 \mathrm{E}+14$ & $1.3923 E+14$ & $1.4361 E+14$ & 5.2373E-02 & 5.3992E-02 & $9.9 \mathrm{E}-01$ & $9.8 \mathrm{E}-01$ \\
\hline $2 s^{2} 2 p^{61} S_{0}$ & $2 s 2 p^{6} 4 p^{1} P_{1}$ & $1.850 \mathrm{E}+14$ & $2.2983 E+14$ & $2.3789 E+14$ & 8.4470E-02 & 8.7389E-02 & $9.9 \mathrm{E}-01$ & $9.8 \mathrm{E}-01$ \\
\hline $2 s^{2} 2 p^{5} 3 s^{3} P_{2}$ & $2 s^{2} 2 p^{5} 3 p{ }^{3} P_{1}$ & $2.917 \mathrm{E}+10$ & $3.0224 E+10$ & $3.0225 E+10$ & $1.8434 \mathrm{E}-02$ & $1.8391 \mathrm{E}-02$ & $9.2 \mathrm{E}-01$ & $1.2 \mathrm{E}+00$ \\
\hline $2 s^{2} 2 p^{5} 3 s^{3} P_{2}$ & $2 s^{2} 2 p^{5} 3 p{ }^{1} P_{1}$ & $2.395 E+11$ & $2.4114 \mathrm{E}+11$ & $2.3979 E+11$ & 1.1881E-02 & 1.1799E-02 & $9.8 \mathrm{E}-01$ & $1.1 \mathrm{E}+00$ \\
\hline $2 s^{2} 2 p^{5} 3 s^{3} P_{2}$ & $2 s^{2} 2 p^{5} 3 p^{3} D_{3}$ & $1.669 \mathrm{E}+12$ & $1.6814 \mathrm{E}+12$ & $1.6674 \mathrm{E}+12$ & 1.9361E-01 & 1.9177E-01 & $1.1 \mathrm{E}-00$ & $1.1 \mathrm{E}+00$ \\
\hline $2 s^{2} 2 p^{5} 3 s^{3} P_{2}$ & $2 s^{2} 2 p^{5} 3 p^{3} P_{2}$ & $9.092 E+11$ & $9.1611 E+11$ & $9.0791 \mathrm{E}+11$ & 7.1771E-02 & 7.1016E-02 & 9.7E-01 & $1.0 \mathrm{E}+00$ \\
\hline $2 s^{2} 2 p^{5} 3 s^{3} P_{2}$ & $2 s 2 p^{6} 3 s^{3} S_{1}$ & $1.580 E+13$ & $1.5536 \mathrm{E}+13$ & $1.4716 \mathrm{E}+13$ & $6.1622 \mathrm{E}-02$ & 5.8201E-02 & $9.5 \mathrm{E}-01$ & 8.6E-01 \\
\hline $2 s^{2} 2 p^{5} 3 s^{3} P_{2}$ & $2 s^{2} 2 p^{5} 4 p{ }^{3} P_{1}$ & $4.805 \mathrm{E}+13$ & $5.4832 E+13$ & $5.5384 E+13$ & $6.6094 \mathrm{E}-02$ & 6.6730E-02 & $1.0 \mathrm{E}-00$ & $1.0 \mathrm{E}+00$ \\
\hline $2 s^{2} 2 p^{5} 3 s^{3} P_{2}$ & $2 s^{2} 2 p^{5} 4 p^{3} D_{2}$ & $2.845 E+13$ & $3.2448 E+13$ & $3.2797 E+13$ & $6.5169 \mathrm{E}-02$ & $6.5840 \mathrm{E}-02$ & $1.0 \mathrm{E}-00$ & $1.0 \mathrm{E}+00$ \\
\hline $2 s^{2} 2 p^{5} 3 s^{3} P_{2}$ & $2 s^{2} 2 p^{5} 4 p^{3} D_{3}$ & $3.484 \mathrm{E}+13$ & $4.0782 E+13$ & $4.1553 E+13$ & $1.0485 \mathrm{E}-01$ & 1.0679E-01 & 9.9E-01 & 9.9E-01 \\
\hline $2 s^{2} 2 p^{5} 3 s^{3} P_{2}$ & $2 s^{2} 2 p^{5} 5 p^{3} P_{1}$ & $2.616 \mathrm{E}+13$ & $2.9775 E+13$ & $3.0477 E+13$ & $1.7587 \mathrm{E}-02$ & 1.7996E-02 & $1.0 \mathrm{E}-00$ & $9.8 \mathrm{E}-01$ \\
\hline $2 s^{2} 2 p^{5} 3 s^{3} P_{2}$ & $2 s^{2} 2 p^{5} 5 p^{3} D_{3}$ & $2.101 E+13$ & $2.4627 E+13$ & $2.5611 E+13$ & 3.2866E-02 & 3.4170E-02 & $9.8 \mathrm{E}-01$ & $9.7 \mathrm{E}-01$ \\
\hline $2 s^{2} 2 p^{5} 3 s^{1} P_{1}$ & $2 s^{2} 2 p^{5} 3 p{ }^{1} P_{1}$ & $1.342 \mathrm{E}+12$ & $1.3518 \mathrm{E}+12$ & $1.3417 \mathrm{E}+12$ & 1.1467E-01 & 1.1362E-01 & $1.0 \mathrm{E}-00$ & $1.1 \mathrm{E}+00$ \\
\hline $2 s^{2} 2 p^{5} 3 s^{1} P_{1}$ & $2 s^{2} 2 p^{5} 3 p{ }^{3} P_{2}$ & $8.614 \mathrm{E}+11$ & $8.6757 E+11$ & $8.6034 E+11$ & 1.1693E-01 & 1.1573E-01 & $1.0 \mathrm{E}-00$ & $1.0 \mathrm{E}+00$ \\
\hline $2 s^{2} 2 p^{5} 3 s^{1} P_{1}$ & $2 s^{2} 2 p^{5} 3 p{ }^{1} S_{0}$ & $2.411 E+12$ & $2.4572 E+12$ & $2.4497 \mathrm{E}+12$ & 5.3889E-02 & 5.3551E-02 & $9.5 \mathrm{E}-01$ & 9.9E-01 \\
\hline $2 s^{2} 2 p^{5} 3 s^{1} P_{1}$ & $2 s 2 p^{6} 3 s^{1} S_{0}$ & $2.171 \mathrm{E}+13$ & $2.1941 E+13$ & $2.1398 E+13$ & 4.6553E-02 & 4.5196E-02 & $9.5 \mathrm{E}-01$ & 8.3E-01 \\
\hline $2 s^{2} 2 p^{5} 3 s^{1} P_{1}$ & $2 s^{2} 2 p^{5} 4 p{ }^{3} D_{2}$ & $2.894 \mathrm{E}+13$ & $3.2979 E+13$ & $3.3351 \mathrm{E}+13$ & 1.1095E-01 & 1.1214E-01 & $9.9 \mathrm{E}-01$ & $9.9 \mathrm{E}-01$ \\
\hline $2 s^{2} 2 p^{5} 3 s^{1} P_{1}$ & $2 s^{2} 2 p^{5} 4 p{ }^{1} P_{1}$ & $2.956 E+13$ & $3.4575 E+13$ & $3.5129 E+13$ & 6.3790E-02 & $6.4783 \mathrm{E}-02$ & $9.9 \mathrm{E}-01$ & $9.8 \mathrm{E}-01$ \\
\hline $2 s^{2} 2 p^{5} 3 s^{1} P_{1}$ & $2 s^{2} 2 p^{5} 4 p{ }^{1} S_{0}$ & $3.309 E+13$ & $3.8940 E+13$ & $3.9514 \mathrm{E}+13$ & 2.3629E-02 & $2.3958 \mathrm{E}-02$ & $1.0 \mathrm{E}-00$ & $1.0 \mathrm{E}+00$ \\
\hline $2 s^{2} 2 p^{5} 3 s^{1} P_{1}$ & $2 s^{2} 2 p^{5} 5 p{ }^{1} S_{0}$ & $2.062 E+13$ & $2.4239 E+13$ & $2.4802 \mathrm{E}+13$ & 7.6916E-03 & $7.8668 \mathrm{E}-03$ & $1.0 \mathrm{E}-00$ & $1.0 \mathrm{E}+00$ \\
\hline
\end{tabular}

TABLE 11. Comprisons of lifetime in Ne-like W

\begin{tabular}{|c|c|c|c|c|c|}
\hline & Label & $\mathrm{J}$ & Lifetime (GRASP) & $\begin{array}{l}\text { Lifetime (MRMP) } \\
\text { [Ref. 34] }\end{array}$ & $\begin{array}{l}\text { Lifetimes } \\
\text { Ref. [24] }\end{array}$ \\
\hline 1 & $2 s^{2} 2 p^{6}{ }^{1} s^{e}$ & 0 & 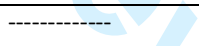 & -------------- & ------------. \\
\hline 2 & $2 s^{2} 2 p^{5} 3 s^{3} p^{0}$ & 2 & $2.326-10$ & $2.667-10$ & $2.31 \mathrm{E}-10$ \\
\hline 3 & $2 s^{2} 2 p^{5} 3 s^{1} p^{0}$ & 1 & $6.532-15$ & $8.291-15$ & $6.48 \mathrm{E}-15$ \\
\hline 4 & $2 s^{2} 2 p^{5} 3 p{ }^{3} p^{e}$ & 1 & $1.534-11$ & $2.038-11$ & $1.53 \mathrm{E}-11$ \\
\hline 5 & $2 s^{2} 2 p^{5} 3 p^{3} D^{e}$ & 2 & $1.042-12$ & $1.186-12$ & $1.04 \mathrm{E}-12$ \\
\hline 6 & $2 s^{2} 2 p^{5} 3 p^{1} P^{e}$ & 1 & $6.236-13$ & $6.313-13$ & $6.28 \mathrm{E}-13$ \\
\hline 7 & $2 s^{2} 2 p^{5} 3 p^{3} D^{e}$ & 3 & $5.947-13$ & $5.991-13$ & $6.00 \mathrm{E}-13$ \\
\hline 8 & $2 s^{2} 2 p^{5} 3 p^{3} p^{e}$ & 2 & $3.707-13$ & $3.875-13$ & $3.72 \mathrm{E}-13$ \\
\hline 9 & $2 s^{2} 2 p^{5} 3 p{ }^{1} s^{e}$ & 0 & $4.069-13$ & $4.148-13$ & $4.08 \mathrm{E}-13$ \\
\hline 10 & $2 s^{2} 2 p^{5} 3 d^{3} p^{0}$ & 0 & $8.081-13$ & $8.091-13$ & $8.19 E-13$ \\
\hline 11 & $2 s^{2} 2 p^{5} 3 d^{3} p^{0}$ & 1 & $1.197-14$ & $1.497-14$ & $1.21 \mathrm{E}-14$ \\
\hline 12 & $2 s^{2} 2 p^{5} 3 d^{3} F^{0}$ & 3 & $7.670-13$ & $7.686-13$ & $7.79 \mathrm{E}-13$ \\
\hline 13 & $2 s^{2} 2 p^{5} 3 d^{3} D^{0}$ & 2 & $7.309-13$ & $7.326-13$ & $7.43 E-13$ \\
\hline 14 & $2 s^{2} 2 p^{5} 3 d^{3} F^{0}$ & 4 & $6.324-12$ & $6.310-12$ & $6.41 \mathrm{E}-12$ \\
\hline 15 & $2 s^{2} 2 p^{5} 3 d{ }^{1} D^{0}$ & 2 & $3.496-12$ & $3.594-12$ & $3.52 \mathrm{E}-12$ \\
\hline 16 & $2 s^{2} 2 p^{5} 3 d^{3} D^{0}$ & 3 & $5.761-12$ & $5.760-12$ & $5.85 \mathrm{E}-12$ \\
\hline 17 & $2 s^{2} 2 p^{5} 3 d{ }^{1} P^{\circ}$ & 1 & $3.561-16$ & $3.827-16$ & $3.59 E-16$ \\
\hline 18 & $2 s^{2} 2 p^{5} 3 s^{3} p^{0}$ & 0 & $3.057-11$ & $3.036-11$ & $3.16 \mathrm{E}-11$ \\
\hline 19 & $2 s^{2} 2 p^{5} 3 s^{3} p^{0}$ & 1 & $2.617-14$ & $3.707-14$ & $2.56 \mathrm{E}-14$ \\
\hline 20 & $2 s^{2} 2 p^{5} 3 p^{3} D^{e}$ & 1 & $1.504-11$ & $1.482-11$ & $1.52 \mathrm{E}-11$ \\
\hline 21 & $2 s^{2} 2 p^{5} 3 p{ }^{3} p^{e}$ & 0 & $3.211-12$ & $3.283-12$ & $3.01 \mathrm{E}-12$ \\
\hline 22 & $2 s 2 p^{6} 3 s^{3} s^{e}$ & 1 & $5.344-14$ & $5.253-14$ & $5.64 \mathrm{E}-14$ \\
\hline 23 & $2 s 2 p^{6} 3 s^{1} s^{e}$ & 0 & $4.521-14$ & $4.571-14$ & $4.64 \mathrm{E}-14$ \\
\hline 24 & $2 s^{2} 2 p^{5} 3 p{ }^{1} D^{e}$ & 2 & $3.538-13$ & $3.717-13$ & $3.55 \mathrm{E}-13$ \\
\hline 25 & $2 s^{2} 2 p^{5} 3 p^{3} s^{e}$ & 1 & $1.332-13$ & $1.433-13$ & $1.27 \mathrm{E}-13$ \\
\hline 26 & $2 s 2 p^{6} 3 p^{3} P^{0}$ & 0 & $4.259-14$ & 4.304-14 & 4.37E-14 \\
\hline 27 & $2 s 2 p^{6} 3 p^{3} P^{0}$ & 1 & $1.252-15$ & $1.547-15$ & $1.22 \mathrm{E}-15$ \\
\hline 28 & $2 s^{2} 2 p^{5} 3 d{ }^{3} F^{0}$ & 2 & $7.336-13$ & $7.346-13$ & $7.44 \mathrm{E}-13$ \\
\hline 29 & $2 s^{2} 2 p^{5} 3 d^{3} D^{0}$ & 1 & $7.343-16$ & $8.130-16$ & $7.53 E-16$ \\
\hline 30 & $2 s^{2} 2 p^{5} 3 d{ }^{3} p^{0}$ & 2 & $3.275-12$ & $3.251-12$ & 3.36E-12 \\
\hline 31 & $2 s^{2} 2 p^{5} 3 d^{1} F^{0}$ & 3 & $5.162-12$ & $5.162-12$ & $5.23 \mathrm{E}-12$ \\
\hline 32 & $2 s 2 p^{6} 3 p^{3} p^{0}$ & 2 & $3.967-14$ & $4.014-14$ & $4.06 \mathrm{E}-14$ \\
\hline 33 & $2 s 2 p^{6} 3 p{ }^{1} P^{0}$ & 1 & $2.150-15$ & $2.780-15$ & $2.13 E-15$ \\
\hline 34 & $2 s 2 p^{6} 3 d^{3} D^{e}$ & 1 & $4.449-14$ & $4.490-14$ & $4.53 \mathrm{E}-14$ \\
\hline 35 & $2 s 2 p^{6} 3 d^{3} D^{e}$ & 2 & $3.454-14$ & $3.571-14$ & $3.51 \mathrm{E}-14$ \\
\hline
\end{tabular}




\begin{tabular}{|c|c|c|c|c|c|}
\hline 36 & $2 s 2 p^{6} 3 d^{3} D^{e}$ & 3 & $4.388-14$ & $4.434-14$ & $4.49 \mathrm{E}-14$ \\
\hline 37 & $2 s 2 p^{6} 3 d^{1} D^{e}$ & 2 & $2.957-14$ & $3.061-14$ & $3.00 \mathrm{E}-14$ \\
\hline 38 & $2 s^{2} 2 p^{5} 4 s^{3} p^{0}$ & 2 & $1.484-14$ & $1.673-14$ & $1.45 \mathrm{E}-14$ \\
\hline 39 & $2 s^{2} 2 p^{5} 4 s^{1} p^{0}$ & 1 & $8.267-15$ & $9.770-15$ & $8.08 \mathrm{E}-15$ \\
\hline 40 & $2 s^{2} 2 p^{5} 4 p^{3} p^{e}$ & 1 & $1.220-14$ & $1.384-14$ & $1.20 \mathrm{E}-14$ \\
\hline 41 & $2 s^{2} 2 p^{5} 4 p{ }^{3} D^{e}$ & 2 & $1.212-14$ & $1.373-14$ & 1.19E-14 \\
\hline 42 & $2 s^{2} 2 p^{5} 4 p^{3} D^{e}$ & 3 & $1.931-14$ & $2.257-14$ & $1.89 \mathrm{E}-14$ \\
\hline 43 & $2 s^{2} 2 p^{5} 4 p{ }^{1} p^{e}$ & 1 & $1.914-14$ & $2.233-14$ & $1.88 \mathrm{E}-14$ \\
\hline 44 & $2 s^{2} 2 p^{5} 4 p^{3} p^{e}$ & 2 & $1.939-14$ & $2.265-14$ & $1.90 \mathrm{E}-14$ \\
\hline 45 & $2 s^{2} 2 p^{5} 4 p{ }^{1} s^{e}$ & 0 & $1.990-14$ & $2.331-14$ & $1.96 \mathrm{E}-14$ \\
\hline 46 & $2 s^{2} 2 p^{5} 4 d^{3} p^{0}$ & 0 & $8.226-15$ & $9.049-15$ & $8.15 E-15$ \\
\hline 47 & $2 s^{2} 2 p^{5} 4 d^{3} p^{0}$ & 1 & $6.484-15$ & $7.245-15$ & $6.46 \mathrm{E}-15$ \\
\hline 48 & $2 s^{2} 2 p^{5} 4 d^{3} F^{0}$ & 3 & $3.259-15$ & $9.084-15$ & $8.18 \mathrm{E}-15$ \\
\hline 49 & $2 s^{2} 2 p^{5} 4 d^{3} D^{0}$ & 2 & $8.289-15$ & $9.119-15$ & $8.22 \mathrm{E}-15$ \\
\hline 50 & $2 s^{2} 2 p^{5} 4 d^{3} F^{0}$ & 4 & $7.933-15$ & $8.479-15$ & $7.88 \mathrm{E}-15$ \\
\hline 51 & $2 s^{2} 2 p^{5} 4 d^{1} D^{0}$ & 2 & $7.930-15$ & $8.475-15$ & $7.88 \mathrm{E}-15$ \\
\hline 52 & $2 s^{2} 2 p^{5} 4 d^{3} D^{0}$ & 3 & $7.977-15$ & $8.526-15$ & 7.93E-15 \\
\hline 53 & $2 s^{2} 2 p^{5} 4 d^{1} p^{0}$ & 1 & $8.793-16$ & $8.788-16$ & 8.83E-16 \\
\hline 54 & $2 s^{2} 2 p^{5} 4 f^{3} D^{e}$ & 1 & $3.739-15$ & $3.870-15$ & $3.75 E-15$ \\
\hline 55 & $2 s^{2} 2 p^{5} 4 f^{3} G^{e}$ & 4 & $3.828-15$ & $3.964-15$ & $3.84 \mathrm{E}-15$ \\
\hline 56 & $2 s^{2} 2 p^{5} 4 f^{3} F^{e}$ & 3 & $3.823-15$ & $3.958-15$ & $3.84 \mathrm{E}-15$ \\
\hline 57 & $2 s^{2} 2 p^{5} 4 f^{3} G^{e}$ & 5 & $3.930-15$ & $4.021-15$ & $3.94 \mathrm{E}-15$ \\
\hline 58 & $2 s^{2} 2 p^{5} 4 f^{1} D^{e}$ & 2 & $3.813-15$ & $3.907-15$ & $3.83 \mathrm{E}-15$ \\
\hline 59 & $2 s^{2} 2 p^{5} 4 f{ }^{1} F^{e}$ & 3 & $3.898-15$ & $3.981-15$ & $3.91 \mathrm{E}-15$ \\
\hline 60 & $2 s^{2} 2 p^{5} 4 f^{3} F^{e}$ & 4 & $3.939-15$ & $4.022-15$ & $3.95 \mathrm{E}-15$ \\
\hline 61 & $2 s^{2} 2 p^{5} 4 s^{3} p^{0}$ & 0 & $1.470-14$ & $1.655-14$ & $1.44 \mathrm{E}-14$ \\
\hline 62 & $2 s^{2} 2 p^{5} 4 s^{3} p^{0}$ & 1 & $1.151-14$ & $1.349-14$ & $1.12 \mathrm{E}-14$ \\
\hline 63 & $2 s^{2} 2 p^{5} 4 p{ }^{3} D^{e}$ & 1 & $1.205-14$ & $1.365-14$ & $1.19 \mathrm{E}-14$ \\
\hline 64 & $2 s^{2} 2 p^{5} 4 p^{3} p^{e}$ & 0 & $1.244-14$ & $1.413-14$ & $1.23 \mathrm{E}-14$ \\
\hline 65 & $2 s^{2} 2 p^{5} 5 s^{3} p^{0}$ & 2 & $1.859-14$ & $2.037-14$ & $1.79 \mathrm{E}-14$ \\
\hline 66 & $2 s^{2} 2 p^{5} 5 s^{1} p^{0}$ & 1 & $1.179-14$ & $1.384-14$ & $1.13 \mathrm{E}-14$ \\
\hline 67 & $2 s^{2} 2 p^{5} 5 p^{3} p^{e}$ & 1 & $1.531-14$ & $1.710-14$ & $1.49 \mathrm{E}-14$ \\
\hline 68 & $2 s^{2} 2 p^{5} 5 p^{3} D^{e}$ & 2 & $1.529-14$ & $1.708-14$ & $1.49 \mathrm{E}-14$ \\
\hline 69 & $2 s^{2} 2 p^{5} 5 p^{1} p^{e}$ & 1 & $2.222-14$ & $2.575-14$ & $2.16 \mathrm{E}-14$ \\
\hline 70 & $2 s^{2} 2 p^{5} 5 p^{3} D^{e}$ & 3 & $2.310-14$ & $2.672-14$ & $2.22 \mathrm{E}-14$ \\
\hline 71 & $2 s^{2} 2 p^{5} 5 p^{3} p^{e}$ & 2 & $2305-14$ & $2.672-14$ & $2.23 \mathrm{E}-14$ \\
\hline 72 & $2 s^{2} 2 p^{5} 5 p{ }^{1} s^{e}$ & 0 & $2.296-14$ & $2.675-14$ & $2.28 \mathrm{E}-14$ \\
\hline 73 & $2 s^{2} 2 p^{5} 4 p{ }^{3} s^{e}$ & 1 & $1.938-14$ & $2.264-14$ & $1.90 \mathrm{E}-14$ \\
\hline 74 & $2 s^{2} 2 p^{5} 4 p{ }^{1} D^{e}$ & 2 & $1.111-14$ & $1.185-14$ & $1.86 \mathrm{E}-14$ \\
\hline 75 & $2 s^{2} 2 p^{5} 5 d^{3} p^{0}$ & 0 & $1.907-14$ & $2.224-14$ & 1.09E-14 \\
\hline 76 & $2 s^{2} 2 p^{5} 5 d^{3} p^{0}$ & 1 & $9.016-15$ & $9.738-15$ & $8.91 \mathrm{E}-15$ \\
\hline 77 & $2 s^{2} 2 p^{5} 5 d^{3} F^{0}$ & 3 & $1.117-14$ & $1.192-14$ & 1.10E-14 \\
\hline 78 & $2 s^{2} 2 p^{5} 5 d^{3} D^{\circ}$ & 2 & $1.117-14$ & $1.192-14$ & 1.10E-14 \\
\hline 79 & $2 s^{2} 2 p^{5} 5 d{ }^{3} F^{\circ}$ & 4 & $1.105-14$ & $1.146-14$ & $1.09 \mathrm{E}-14$ \\
\hline 80 & $2 s^{2} 2 p^{5} 5 d^{1} D^{\circ}$ & 2 & $1.097-14$ & $1.139-14$ & $1.08 \mathrm{E}-14$ \\
\hline 81 & $2 s^{2} 2 p^{5} 5 d^{3} D^{\circ}$ & 3 & $1.100-14$ & $1.143-14$ & $1.09 \mathrm{E}-14$ \\
\hline 82 & $2 s^{2} 2 p^{5} 5 d^{1} p^{0}$ & 1 & $3.051-15$ & $3.063-15$ & $3.10 \mathrm{E}-15$ \\
\hline 83 & $2 s^{2} 2 p^{5} 5 f^{3} D^{e}$ & 1 & $7.203-15$ & $7.151-15$ & $7.16 \mathrm{E}-15$ \\
\hline 84 & $2 s^{2} 2 p^{5} 5 f^{3} G^{e}$ & 4 & $7.299-15$ & $7.252-15$ & $7.29 \mathrm{E}-15$ \\
\hline 85 & $2 s^{2} 2 p^{5} 5 f{ }^{3} F^{e}$ & 2 & $7.205-15$ & $7.164-15$ & $7.18 \mathrm{E}-15$ \\
\hline 86 & $2 s^{2} 2 p^{5} 5 f^{3} F^{e}$ & 3 & $7.257-15$ & $7.214-15$ & $7.24 \mathrm{E}-15$ \\
\hline 87 & $2 s^{2} 2 p^{5} 5 f^{3} G^{e}$ & 5 & $7.519-15$ & $7.352-15$ & $7.49 \mathrm{E}-15$ \\
\hline 88 & $2 s^{2} 2 p^{5} 5 f^{1} D^{e}$ & 2 & $7.250-15$ & $7.132-15$ & $7.28 \mathrm{E}-15$ \\
\hline 89 & $2 s^{2} 2 p^{5} 5 f^{1} F^{e}$ & 3 & $7.457-15$ & $7.301-15$ & $7.43 \mathrm{E}-15$ \\
\hline 90 & $2 s^{2} 2 p^{5} 5 f^{3} F^{e}$ & 4 & $7.492-15$ & $7.340-15$ & $7.48 \mathrm{E}-15$ \\
\hline 91 & $2 s^{2} 2 p^{5} 5 g \quad{ }^{3} F^{0}$ & 2 & $1.271-14$ & & $1.27 \mathrm{E}-14$ \\
\hline 92 & $2 s^{2} 2 p^{5} 5 g{ }^{3} H^{0}$ & 5 & $1.283-14$ & & $1.28 \mathrm{E}-14$ \\
\hline 93 & $2 s^{2} 2 p^{5} 5 g{ }^{3} G^{0}$ & 3 & $1.286-14$ & & $1.29 \mathrm{E}-14$ \\
\hline 94 & $2 s^{2} 2 p^{5} 5 g{ }^{3} G^{0}$ & 4 & $1.284-14$ & & $1.28 \mathrm{E}-14$ \\
\hline 95 & $2 s^{2} 2 p^{5} 5 g^{1} F^{0}$ & 3 & $1.305-14$ & & 1.30E-14 \\
\hline 96 & $2 s^{2} 2 p^{5} 5 g{ }^{3} H^{\circ}$ & 6 & $1.298-14$ & & 1.30E-14 \\
\hline 97 & $2 s^{2} 2 p^{5} 5 g \quad{ }^{1} G^{0}$ & 4 & $1.302-14$ & & $1.30 \mathrm{E}-14$ \\
\hline 98 & $2 s^{2} 2 p^{5} 5 g{ }^{3} G^{\circ}$ & 5 & $1.299-14$ & & $1.30 \mathrm{E}-14$ \\
\hline
\end{tabular}




\begin{tabular}{|c|c|c|c|c|c|}
\hline 99 & $2 s^{2} 2 p^{5} 4 d^{3} F^{0}$ & 2 & $8.343-15$ & 9.096-15 & $8.26 \mathrm{E}-15$ \\
\hline 100 & $2 s^{2} 2 p^{5} 4 d{ }^{3} D^{0}$ & 1 & $9.888-16$ & $1.022-15$ & $9.92 \mathrm{E}-16$ \\
\hline 101 & $2 s^{2} 2 p^{5} 4 d{ }^{3} p^{0}$ & 2 & $7.913-15$ & $8.447-15$ & $7.87 \mathrm{E}-15$ \\
\hline 102 & $2 s^{2} 2 p^{5} 4 d^{1} F^{0}$ & 3 & $7.955-15$ & 3.505-15 & $7.91 \mathrm{E}-15$ \\
\hline 103 & $2 s^{2} 2 p^{5} 4 f^{3} G^{e}$ & 3 & $3.822-15$ & 3.959-15 & 3.84E-15 \\
\hline 104 & $2 s^{2} 2 p^{5} 4 f{ }^{3} D^{e}$ & 2 & & & $3.78 \mathrm{E}-15$ \\
\hline 105 & $2 s^{2} 2 p^{5} 4 f{ }^{3} D^{e}$ & 3 & $3.907-15$ & $3.992-15$ & $3.92 \mathrm{E}-15$ \\
\hline 106 & $2 s^{2} 2 p^{5} 4 f{ }^{1} G^{e}$ & 4 & $3.935-15$ & 4.021-15 & 3.95E-15 \\
\hline 107 & $2 s 2 p^{6} 4 s{ }^{3} s^{e}$ & 1 & $1.096-14$ & $1.198-14$ & $1.09 \mathrm{E}-14$ \\
\hline 108 & $2 s 2 p^{6} 4 s^{1} s^{e}$ & 0 & $1.102-14$ & $1.206-14$ & $1.09 \mathrm{E}-14$ \\
\hline 109 & $2 s 2 p^{6} 4 p{ }^{3} p^{0}$ & 0 & $9.513-15$ & $1.051-15$ & $9.45 \mathrm{E}-15$ \\
\hline 110 & $2 s 2 p^{6} 4 p{ }^{3} p^{0}$ & 1 & $4.084-15$ & 4.824-15 & 4.00E-15 \\
\hline 111 & $2 s 2 p^{6} 4 p{ }^{3} p^{0}$ & 2 & $1.337-14$ & $1.491-14$ & $1.32 \mathrm{E}-14$ \\
\hline 112 & $2 s 2 p^{6} 4 p{ }^{1} p^{0}$ & 1 & $3.276-15$ & $3.957-15$ & 3.19E-15 \\
\hline 113 & $2 s 2 p^{6} 4 d^{3} D^{e}$ & 1 & $6.918-15$ & 7.505-15 & $6.89 \mathrm{E}-15$ \\
\hline 114 & $2 s 2 p^{6} 4 d^{3} D^{e}$ & 2 & $6.880-15$ & 7.454-15 & $\begin{array}{l}0.095-13 \\
6.85 E-15\end{array}$ \\
\hline 115 & $2 s 2 p^{6} 4 d^{3} D^{e}$ & 3 & $6.692-15$ & 7.087-15 & $6.67 \mathrm{E}-15$ \\
\hline 116 & $2 s 2 p^{6} 4 d^{1} D^{e}$ & 2 & $6.616-15$ & $6.987-15$ & $6.60 \mathrm{E}-15$ \\
\hline 117 & $2 s 2 p^{6} 4 f^{3} F^{\circ}$ & 2 & $3.513-15$ & $3.648-15$ & 3.51E-15 \\
\hline 118 & $2 s 2 p^{6} 4 f{ }^{3} F^{\circ}$ & 3 & $3.519-15$ & $3.640-15$ & $3.52 \mathrm{E}-15$ \\
\hline 119 & $2 s 2 p^{6} 4 f{ }^{3} F^{\circ}$ & 4 & $3.602-15$ & $3.681-15$ & 3.60E-15 \\
\hline 120 & $2 s 2 p^{6} 4 f{ }^{1} F^{\circ}$ & 3 & $3.617-15$ & $3.707-15$ & 3.61E-15 \\
\hline
\end{tabular}

